\title{
An algorithmic approach to the multiple impact of a disk in a corner.
}

\author{
Claudia Fassino, Stefano Pasquero ${ }^{\dagger}$
}

February 15, 2019

\begin{abstract}
We present the algorithmic procedure determining the impulsive behavior of a rigid disk having a single or possibly multiple frictionless impact with two walls forming a corner. The algorithmic procedure represents an application of the general theory of multiple impacts as presented in [1] for the ideal case.

In the first part, two theoretical algorithms are presented for the cases of ideal impact and Newtonian frictionless impact with global dissipation index. The termination analysis of the algorithms differentiates the two cases: in the ideal case, we show that the algorithm always terminates and the disk exits from the corner after a finite number of steps independently of the initial impact velocity of the disk and the angle formed by the walls; in the non-ideal case, although is not proved that the disk exits from the corner in a finite number of steps, we show that its velocity decreases to zero and the termination of the algorithm can be fixed through an "almost at rest" condition.

In the second part, we present a numerical version of both the theoretical algorithms that is more robust than the theoretical ones with respect to noisy initial data and floating point arithmetic computation. Moreover, we list and analyze the outputs of the numerical algorithm in several cases.
\end{abstract}

2010 Mathematical subject classification: 70E18; 70F35; 70-04

Keywords: Multipoint Impact - Iterative Method

\footnotetext{
${ }^{*}$ Department of Mathematics, University of Genova - Via Dodecaneso 35, 16146 GENOVA - email: fassino@dima.unige.it

${ }^{\dagger}$ Department of Mathematical, Physical and Computer Sciences, University of Parma. Parco Area delle Scienze 53/a (Campus) 43124 PARMA. email: stefano.pasquero@unipr.it
} 


\section{Introduction}

The study of the behavior of a rigid or multibody system subject to multiple contact and/or impact is a very actual argument of investigation, finding application in several branches of Classical Mechanics, from the analysis of the motion of billiard balls to that of rocking blocks or granular materials.

The argument can be dealt following several different approaches, ranging from completely theoretical to specifically numerical (see e.g. [2] for a wide but not complete bibliography).

In a recent paper [1], a geometric approach framed in the context of jet-bundle theory was used to analyze the behavior of a general mechanical system with a finite number of degrees of freedom subject to multiple unilateral ideal constraints. The analysis, simply based on very general arguments of preservation of the kinetic energy, led to the construction of a theoretical algorithm that determines the right velocity of the system for several significant mechanical systems once the left velocity and the geometric properties of the system are known.

In this paper we present the application of this algorithm to the paradigmatic case of the planar system formed by a rigid disk simultaneously impacting with both sides of a corner in two possible situations: the ideal case, with frictionless contacts and conservation of the kinetic energy of the disk; the so called Newtonian frictionless impact with global dissipation index, a non-ideal case with frictionless contact and no requirement of conservation of kinetic energy.

In the ideal case, the algorithm is directly built on the theoretical results of [1], and the main result, apart from the analysis of the physical meaning of the output, pertains to the termination analysis. This is an important aspect of the approach that was not discussed in [1]: although for several meaningful systems the theoretical algorithm evidently terminates, it is clear that the requirement of conservation of kinetic energy suggests the possibility of an infinite number of iterations of the algorithm, reflecting a possible infinite number of rebounds of the disk. We prove that the algorithm always terminates and determines a velocity such that the disk exits from the corner. However, notwithstanding the manifest simplicity of the mechanical system, the analysis lights up two important aspects of multiple ideal impacts: the first is that the geometry of the system can be easily arranged in order to obtain as many iterations of the algorithm as one can decide; the second is that the case of effective double impact with both the sides of the corner, although leading to a non trivial non-linear rule for the determination of the right velocity, is however such that that the double impact can happen only once.

The algorithm for the non-ideal case consists in a generalization of the ideal one supposing that the (frictionless) walls of the corner are partially or totally inelastic. The non-ideality is introduced by a Newtonian restitution 
coefficient $\varepsilon$ relating the orthogonal components of the velocity of the disk with respect to the walls before and after each step of the algorithm. In this case we do not prove that the algorithm always determines a velocity such that the disk exits from the corner, but we show that the norm of the velocity decreases to 0 for increasing numbers of steps. This gives a second termination criterion for the algorithm, with a meaningful physical interpretation. However, even in the non-ideal case, we prove that double impacts of the disk with both the sides of the corner can happen only once.

It is however well known that the data derived from real-world measurements can be perturbed by errors, so that theoretical algorithms that process such data can produce unreliable results. Moreover, when an algorithm is implemented, because of the floating point arithmetic, the computed values can be perturbed by algorithmic errors. In case of pedestrian implementation of the theoretical algorithms, small perturbations of the data can cause structural changes in response, consequent different choices in the iterative method, and than invalidate the final results.

For this reason we present a numerical algorithm, based on the theoretical ones, that, differently to these ones, is robust with respect to the errors introduced by the measurements and by the use of the floating point arithmetic. This algorithm is obtained introducing suitable thresholds changing the tests for the choice of the iterative step to do.

The paper is then divided into two main parts: in the first, after some short preliminaries, we introduce the theoretical algorithm for the ideal impact in three different but equivalent forms and we show the corresponding results. In particular we prove that the disk exits from the corner after a finite number of steps independently of the initial impact velocity of the disk and the angle formed by the walls. Then we introduce the theoretical algorithm for the non-ideal impact in two different but equivalent forms and we show the corresponding results. In particular, although we does not prove that disk exits from the corner after a finite number of steps, we prove that its velocity decreases to zero and the termination of the algorithm can be fixed through an "almost at rest" condition.

In the second part we introduce the single numerical algorithm that groups both the ideal and the non-ideal theoretical cases. The numerical algorithm differs from the theoretical ones in the criteria about the velocity that select the behavior of the disk after a rebound. In the theoretical versions such criteria are based on the on the nullity of some suitable components of the velocity. It is however well known that an exact comparison with zero makes the algorithm unstable, and so, in order to obtain a more robust algorithm, we introduce two thresholds: one determining when a single component of the velocity of the disk is almost zero, one determining when the norm of the whole velocity is almost zero. We compare the theoretical and numerical versions of the algorithms showing that, starting from the 
same input, they compute the same output in the same number of steps, or they compute slightly different outputs, even if one of the versions performs more steps. Finally, we illustrate the behavior of the numerical algorithm by listing the outputs computed processing several different meaningful inputs.

Since the main aim of the paper is focused on the analysis of the algorithms giving the velocity of the disk after the impact, we relegate to the appendix a brief but exhaustive sketch of the geometric method determining the constitutive characterizations of the multiple constraint that are the bases for the construction of the algorithms. The Reader interested in a wider description of the geometry and the impulsive aspects of general systems subject to ideal multiple constraints can refer to [1] and the references therein. In the bulk of the paper, we will limit the mathematical aspects to the bare necessary to describe the algorithms for the case of the disk in the corner.

The list of possible references about multiple impacts is very huge, and a bibliography claiming to be exhaustive on the argument should be excessively long compared to the length of the paper. Moreover, only few works would be reasonably pertinent to the specific algorithm presented in the paper. Therefore, the list of references has been based on the minimality criterion of making the paper self-consistent. However, for large but not recent or exhaustive lists of general references, see for example [2, 3, 4, 5, 6, 7].

\section{PART 1: THEORETICAL ASPECTS}

\section{Preliminaries}

A rigid disk of unitary mass and radius $R$ moves in the part of a horizontal plane delimited by two walls $\mathcal{S}_{1}, \mathcal{S}_{2}$ forming an angle $2 \alpha \in(0, \pi)$ (see Fig. 1 ). We can describe the geometry of the system by introducing local coordinates $(x, y, \vartheta)$ where $x, y$ are the coordinates of the center of the disk and $\vartheta$ is the orientation of the disk. Choosing $k=\tan \alpha$, then $k>0$ and the walls can be described by the cartesian relations $\mathcal{S}_{1}: k x-y=0, \mathcal{S}_{2}: k x+y=0$. Since we adopt the so called event-driven approach, we assume that $(x, y)=$ $\left(-\frac{R}{\sin \alpha}, 0\right)$ so that the disk is in contact with both the walls. We assume the contacts as frictionless.

The kinetic state of the disk is known once the linear velocity of the (center of the) disk is assigned through a pair $\mathbf{v}=(\dot{x}, \dot{y})$, and the spin is assigned by $\dot{\vartheta}$. Of course, the linear velocity can be assigned in the alternative form $(v \cos \varphi, v \sin \varphi)$ with $(v>0$ and $) \varphi \in(-\pi, \pi]$. Without entering temporarily into mathematical details, it is clear that the angle $\varphi$ determines the nature of the impact between disk and walls: if $\varphi \in(-\alpha, \alpha)$, the disk is subject to a multiple impact, if $\varphi \in[\alpha, \pi-\alpha)$ the disk is subject to an 


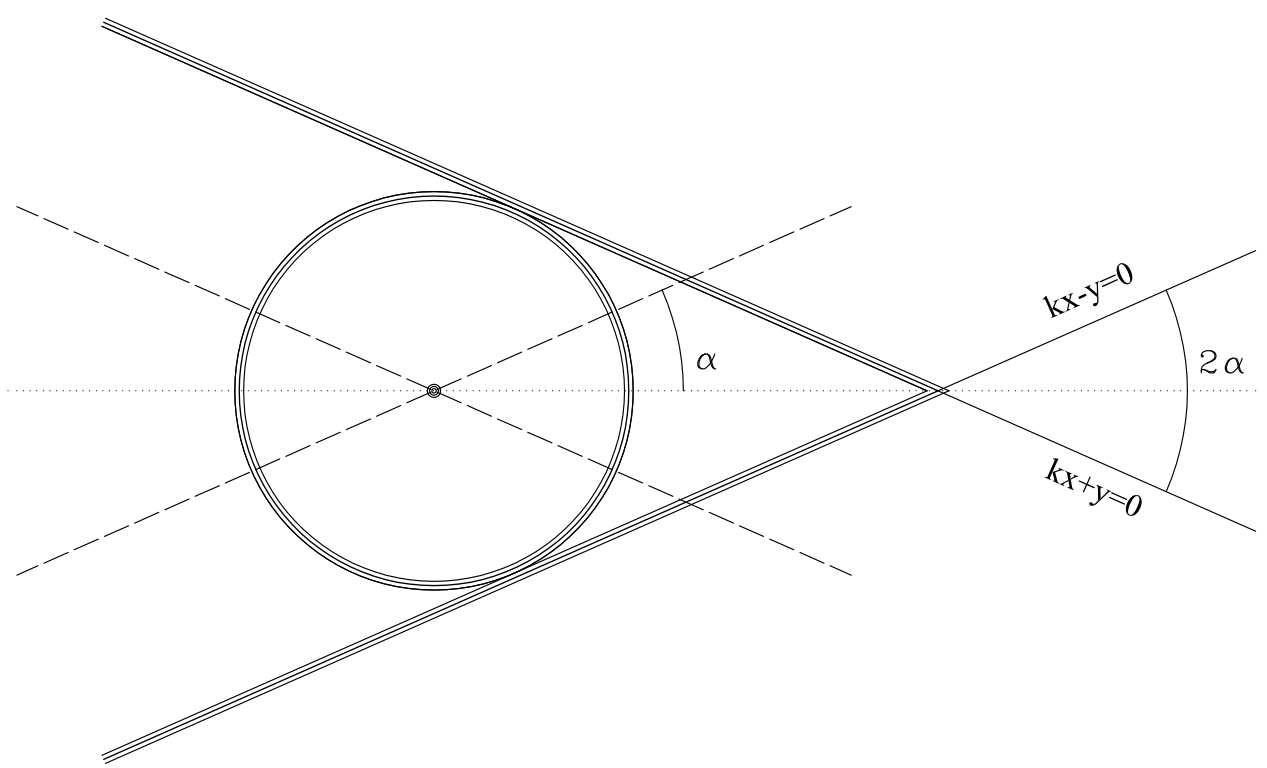

Figure 1: Disk in contact with both sides of the corner

impact with $\mathcal{S}_{2}$ and not with $\mathcal{S}_{1}$, if $\varphi \in(-\pi+\alpha,-\alpha]$ the disk is subject to an impact with $\mathcal{S}_{1}$ and not with $\mathcal{S}_{2}$. Otherwise, the velocity $\mathbf{v}$ is a so-called exit velocity, the disk does not impact with the walls and its time evolution separates it from one or both the walls.

We can divide the space of the linear velocity of the system in four different zones $\mathcal{Z}_{0}, \mathcal{Z}_{1}, \mathcal{Z}_{2}, \mathcal{Z}_{12}$ with $\mathbf{v} \in \mathcal{Z}_{i}$ if, with a slight abuse of notation, the linear velocity determines an impact with $\mathcal{S}_{i}$.

REMARK 1. If we restrict our attention to a physically meaningful situation and taking into account Fig. ??, the initial velocity $\mathbf{v}_{0}=\left(\dot{x}_{0}, \dot{y}_{0}\right)$ is such that $\mathbf{v}_{0} \in \mathcal{Z}_{12}$ or $\mathbf{v}_{0}$ is parallel to the walls, that is $\varphi_{0} \in[-\alpha, \alpha]$.

We now resume the main consequences of the constitutive characterization of multiple contact/impact presented in [1] and sketched in the Appendix for the case of the disk in the corner:

0) the constitutive characterization assigns a reactive impulse $\mathbf{I}$ to any kinetic state of the disk supposed in contact with the walls;

1) independently of the kinetic state of the disk, the reactive impulse $\mathbf{I}$ does not involve the spin $\dot{\vartheta}$ of the disk, that remains unchanged in the impact. This is coherent with the assumption that the contacts of the disk with $\mathcal{S}_{1}$ and $\mathcal{S}_{2}$ are frictionless. Then we can focus our attention only on the linear part $\mathbf{v}$ of the velocity of the disk;

2) for every linear velocity $\mathbf{v}$ we can determine the orthogonal components $\mathbf{v}_{1}^{\perp}(\mathbf{v}), \mathbf{v}_{2}^{\perp}(\mathbf{v})$ of $\mathbf{v}$ with respect to $\mathcal{S}_{1}, \mathcal{S}_{2}$ respectively. The orthogonal 
components indicate if $\mathbf{v}$ is an exit velocity or if $\mathbf{v}$ gives rise to an impact with $\mathcal{S}_{1}$ and/or $\mathcal{S}_{2}$. In particular, if $\mathbf{v}=(\dot{x}, \dot{y})$, we have that:

$$
\begin{aligned}
& \mathbf{v} \in \mathcal{Z}_{0} \Leftrightarrow\left\{\begin{array}{l}
k \dot{x}+\dot{y} \leq 0 \\
k \dot{x}-\dot{y} \leq 0
\end{array}\right. \\
& \mathbf{v} \in \mathcal{Z}_{1} \Leftrightarrow\left\{\begin{array}{l}
k \dot{x}+\dot{y} \leq 0 \\
k \dot{x}-\dot{y}>0
\end{array}\right. \\
& \mathbf{v} \in \mathcal{Z}_{2} \Leftrightarrow\left\{\begin{array}{l}
k \dot{x}+\dot{y}>0 \\
k \dot{x}-\dot{y} \leq 0
\end{array}\right. \\
& \mathbf{v} \in \mathcal{Z}_{12} \Leftrightarrow\left\{\begin{array}{l}
k \dot{x}+\dot{y}>0 \\
k \dot{x}-\dot{y}>0
\end{array}\right.
\end{aligned}
$$

Note that the symmetry of the mechanical problem is reflected in the symmetry of the zones $\mathcal{Z}_{i}$ with respect to the $(\dot{x}, \dot{y})$ components of the velocity. In fact we have

$$
\begin{array}{lll}
\left(\dot{x}_{n}, \dot{y}_{n}\right) \in \mathcal{Z}_{0} & \Rightarrow \quad\left(\dot{x}_{n},-\dot{y}_{n}\right) \in \mathcal{Z}_{0} \\
\left(\dot{x}_{n}, \dot{y}_{n}\right) \in \mathcal{Z}_{1} \quad \Rightarrow \quad\left(\dot{x}_{n},-\dot{y}_{n}\right) \in \mathcal{Z}_{2} \\
\left(\dot{x}_{n}, \dot{y}_{n}\right) \in \mathcal{Z}_{2} \quad \Rightarrow \quad\left(\dot{x}_{n},-\dot{y}_{n}\right) \in \mathcal{Z}_{1} \\
\left(\dot{x}_{n}, \dot{y}_{n}\right) \in \mathcal{Z}_{12} \quad \Rightarrow \quad\left(\dot{x}_{n},-\dot{y}_{n}\right) \in \mathcal{Z}_{12} .
\end{array}
$$

3) the constitutive characterization determines a rule assigning a "new" velocity of the system once an "old" velocity is known. The rule is $\mathbf{v}_{\text {new }}=\mathbf{v}_{\text {old }}+\mathbf{I}\left(\mathbf{v}_{\text {old }}\right)$, where $\mathbf{I}$ represents the reactive impulse generated by the impact with the walls, and the function $\mathbf{I}\left(\mathbf{v}_{\text {old }}\right)$ depends on the ideal or non-ideal nature of the impact.

Of course $\mathbf{I}\left(\mathbf{v}_{\text {old }}\right)=0$ if $\mathbf{v} \in \mathcal{Z}_{0}$ : in this case, the reactive impulse acting on the disk is null and the kinetic energy is obviously preserved, as well as the euclidean norm $\|\mathbf{v}\|_{2}=\sqrt{\dot{x}^{2}+\dot{y}^{2}}$.

The rule $\mathbf{I}=\mathbf{I}\left(\mathbf{v}_{\text {old }}\right)$ consists in a complete or partial "reflection" of the orthogonal component $\mathbf{v}_{i}^{\perp}\left(\mathbf{v}_{\text {old }}\right)$ if $\mathbf{v}_{\text {old }} \in \mathcal{Z}_{i}, i=1,2$. In the ideal case, the reactive impulse acting on the disk has the form $\mathbf{I}\left(\mathbf{v}_{\text {old }}\right)=$ $-2 \mathbf{v}_{i}^{\perp}\left(\mathbf{v}_{\text {old }}\right)$, so that the reflection of the component $\mathbf{v}_{i}^{\perp}\left(\mathbf{v}_{\text {old }}\right)$ is complete. The kinetic energy and $\|\mathbf{v}\|_{2}$ are once again preserved. In the non-ideal case, the reactive impulse acting on the disk has the form $\mathbf{I}\left(\mathbf{v}_{\text {old }}\right)=-(1+\varepsilon) \mathbf{v}_{i}^{\perp}\left(\mathbf{v}_{\text {old }}\right)$ with $0 \leq \varepsilon<1$, so that the reflection of the component $\mathbf{v}_{i}^{\perp}\left(\mathbf{v}_{\text {old }}\right)$ is only partial. The kinetic energy of the system is not preserved and $\left\|\mathbf{v}_{\text {new }}\right\|_{2}<\left\|\mathbf{v}_{\text {old }}\right\|_{2}$.

The rule $\mathbf{I}=\mathbf{I}\left(\mathbf{v}_{\text {old }}\right)$ consists in a strongly non-linear relation in the components of $\mathbf{v}_{\text {old }}$ in case of multiple impact $\mathbf{v}_{\text {old }} \in \mathcal{Z}_{12}$ (see (2d) 
and (14d) below): in this case, the reactive impulse has the form $\mathbf{I}\left(\mathbf{v}_{\text {old }}\right)=\lambda\left(\mathbf{v}_{1}^{\perp}\left(\mathbf{v}_{\text {old }}\right)+\mathbf{v}_{2}^{\perp}\left(\mathbf{v}_{\text {old }}\right)\right)$ where $\lambda$ is a suitable coefficient fit to obtain the conservation of the kinetic energy in the ideal multiple impact (see [1]) or involving the Newtonian restitution coefficient $\varepsilon$ in the non-ideal one;

4) the iterative application of the rule determines an algorithm fit to determine if and how the disk exits from the corner, loosing contact with one or both the walls. The output of the algorithms must become constant if the "new" velocity belongs to the $\mathcal{Z}_{0}$ zone. Then, both in the ideal and the non-ideal cases, the termination analysis of the algorithm is necessarily related to the condition $\mathbf{v} \in \mathcal{Z}_{0}$ (or, of course, on the number of steps). In the non-ideal case, the termination analysis can be also based on the condition $\|\mathbf{v}\|_{2} \leq$ constant upon proof that $\lim _{n \rightarrow+\infty}\|\mathbf{v}\|_{2}=0$.

\section{Theoretical algorithm for the ideal case $\left(\mathrm{TA}_{i d}\right)$}

In this section we present the iterative rule assigning the "new" velocity $\mathbf{v}_{n+1}$ of the disk as function of the "old" velocity $\mathbf{v}_{n}$ for the ideal case in three different forms. Each one of the forms will be used to obtain theoretical results about the algorithm.

\subsection{First expression of TA $_{i d}:$ use of $(\dot{x}, \dot{y})$}

Given an initial velocity $\mathbf{v}_{0}=\left(\dot{x}_{0}, \dot{y}_{0}\right)$, the iterative rule determined by the constitutive characterization of [1] is such that:

If $\left(\dot{x}_{n}, \dot{y}_{n}\right) \in \mathcal{Z}_{0}$, that is if $\left\{\begin{array}{l}k \dot{x}_{n}+\dot{y}_{n} \leq 0 \\ k \dot{x}_{n}-\dot{y}_{n} \leq 0\end{array}\right.$, , then $\left\{\begin{array}{l}\dot{x}_{n+1}=\dot{x}_{n} \\ \dot{y}_{n+1}=\dot{y}_{n}\end{array}\right.$

If $\left(\dot{x}_{n}, \dot{y}_{n}\right) \in \mathcal{Z}_{1}$, that is if $\left\{\begin{array}{l}k \dot{x}_{n}+\dot{y}_{n} \leq 0 \\ k \dot{x}_{n}-\dot{y}_{n}>0\end{array}\right.$, then $\left\{\begin{array}{l}\dot{x}_{n+1}=\frac{1-k^{2}}{1+k^{2}} \dot{x}_{n}+\frac{2 k}{1+k^{2}} \dot{y}_{n} \\ \dot{y}_{n+1}=\frac{2 k}{1+k^{2}} \dot{x}_{n}-\frac{1-k^{2}}{1+k^{2}} \dot{y}_{n}\end{array}\right.$

If $\left(\dot{x}_{n}, \dot{y}_{n}\right) \in \mathcal{Z}_{2}$, that is if $\left\{\begin{array}{l}k \dot{x}_{n}+\dot{y}_{n}>0 \\ k \dot{x}_{n}-\dot{y}_{n} \leq 0\end{array}\right.$, then $\left\{\begin{array}{l}\dot{x}_{n+1}=\frac{1-k^{2}}{1+k^{2}} \dot{x}_{n}-\frac{2 k}{1+k^{2}} \dot{y}_{n} \\ \dot{y}_{n+1}=-\frac{2 k}{1+k^{2}} \dot{x}_{n}-\frac{1-k^{2}}{1+k^{2}} \dot{y}_{n}\end{array}\right.$ 
If $\left(\dot{x}_{n}, \dot{y}_{n}\right) \in \mathcal{Z}_{12}$, that is if $\left\{\begin{array}{l}k \dot{x}_{n}+\dot{y}_{n}>0 \\ k \dot{x}_{n}-\dot{y}_{n}>0\end{array}\right.$, then $\left\{\begin{array}{l}\dot{x}_{n+1}=\frac{-k^{4} \dot{x}_{n}^{2}+\left(1-2 k^{2}\right) \dot{y}_{n}^{2}}{k^{4} \dot{x}_{n}^{2}+\dot{y}_{n}^{2}} \dot{x}_{n} \\ \dot{y}_{n+1}=\frac{k^{2}\left(k^{2}-2\right) \dot{x}_{n}^{2}-\dot{y}_{n}^{2}}{k^{4} \dot{x}_{n}^{2}+\dot{y}_{n}^{2}} \dot{y}_{n}\end{array}\right.$

REMARK 2. A straightforward calculation shows that independently of the condition $\mathbf{v}_{n} \in \mathcal{Z}_{i}$ with $i=0,1,2,12$, we have

$$
\frac{\left(\left\|\mathbf{v}_{n+1}\right\|_{2}\right)^{2}}{\left(\left\|\mathbf{v}_{n}\right\|_{2}\right)^{2}}=\frac{\dot{x}_{n+1}^{2}+\dot{y}_{n+1}^{2}}{\dot{x}_{n}^{2}+\dot{y}_{n}^{2}}=1 .
$$

This is an easily predictable but not trivial consequence of the preservation of the kinetic energy required in [1. In fact, since the kinetic energy is not an absolute quantity but it depends on the choice of a frame of reference, the validity of (3) follows from the nature itself of the contact/impact, that does not affect the angular coordinate of the disk, and the nature itself of the constraint and its set of rest frames. Moreover, $\|\mathbf{v}\|_{2}$ is not the norm of the velocity vector of the disk but only the Euclidean norm of the pair $(\dot{x}, \dot{y})$ viewed as an element of $\mathbb{R}^{2}$ (see Appendix for details).

REMARK 3. The iterative rule (2a 2d d respects the symmetry of the mechanical problem with respect to the $(\dot{x}, \dot{y})$ components of the velocity. In fact an easy calculation shows that, if $\left(\dot{x}_{n}, \dot{y}_{n}\right) \notin \mathcal{Z}_{0}$, then

$$
\left\{\begin{array}{l}
\dot{x}_{n+1}\left(\dot{x}_{n},-\dot{y}_{n}\right)=\dot{x}_{n+1}\left(\dot{x}_{n}, \dot{y}_{n}\right) \\
\dot{y}_{n+1}\left(\dot{x}_{n},-\dot{y}_{n}\right)=-\dot{y}_{n+1}\left(\dot{x}_{n}, \dot{y}_{n}\right)
\end{array}\right.
$$

\subsection{Second expression of $\mathbf{T A}_{i d}$ : use of $(\cos \varphi, \sin \varphi)$}

The same algorithm can be expressed using $\mathbf{v}_{0}$ in the form $\left(v_{0} \cos \varphi_{0}, v_{0} \sin \varphi_{0}\right)$ with $v_{0}>0, \varphi \in(\pi, \pi]$. Since $k=\tan \alpha$ so that

$$
\left\{\begin{array}{l}
\frac{1-k^{2}}{1+k^{2}}=\cos 2 \alpha \\
\frac{2 k}{1+k^{2}}=\sin 2 \alpha
\end{array}\right.
$$

we immediately obtain that the matrices of the linear transformations given by (2b 2c ) are orthogonal but not special orthogonal. Moreover, thanks to (3), $v_{0}$ is factorized in every term. The iterative rule becomes

If $\varphi_{n} \in \mathcal{Z}_{0}, \quad$ that is if $\left\{\begin{array}{l}\cos \varphi_{n}<0 \\ \left|\tan \varphi_{n}\right| \leq \tan \alpha\end{array}\right.$, then $\quad \varphi_{n+1}=\varphi_{n}$ 
If $\varphi_{n} \in \mathcal{Z}_{1}, \quad$ that is if $\left\{\begin{array}{l}\sin \varphi_{n}<0 \\ -\cot \alpha<\cot \varphi_{n} \leq \cot \alpha\end{array}\right.$, then $\quad \varphi_{n+1}=-\varphi_{n}+2 \alpha(5 \mathrm{~b})$

If $\varphi_{n} \in \mathcal{Z}_{2}, \quad$ that is if $\left\{\begin{array}{l}\sin \varphi_{n}>0 \\ -\cot \alpha<\cot \varphi_{n} \leq \cot \alpha\end{array}\right.$, then $\varphi_{n+1}=-\varphi_{n}-2 \alpha(5 \mathrm{c})$

If $\varphi_{n} \in \mathcal{Z}_{12}$, that is if $\left\{\begin{array}{l}\cos \varphi_{n}>0 \\ \left|\tan \varphi_{n}\right|<\tan \alpha\end{array}\right.$, then

$$
\left\{\begin{array}{l}
\cos \varphi_{n+1}=-\left(\frac{\tan ^{4} \alpha \cos ^{2} \varphi_{n}-\sin ^{2} \varphi_{n}}{\tan ^{4} \alpha \cos ^{2} \varphi_{n}+\sin ^{2} \varphi_{n}}+2 \frac{\tan ^{2} \alpha \sin ^{2} \varphi_{n}}{\tan ^{4} \alpha \cos ^{2} \varphi_{n}+\sin ^{2} \varphi_{n}}\right) \cos \varphi_{n} \\
\sin \varphi_{n+1}=\left(\frac{\tan ^{4} \alpha \cos ^{2} \varphi_{n}-\sin ^{2} \varphi_{n}}{\tan ^{4} \alpha \cos ^{2} \varphi_{n}+\sin ^{2} \varphi_{n}}-2 \frac{\tan ^{2} \alpha \cos ^{2} \varphi_{n}}{\tan ^{4} \alpha \cos ^{2} \varphi_{n}+\sin ^{2} \varphi_{n}}\right) \sin \varphi_{n}
\end{array}\right.
$$

\subsection{Third expression of TA $_{i d}$ : use of $(\dot{\xi}, \dot{\eta})$}

A third version of the algorithm can be obtained by using a standard change of coordinates $(\xi, \eta)=(k x+y, k x-y)$ that identifies the velocity using its projections in the directions of the walls. Then we have

$$
\left\{\begin{array} { l } 
{ \dot { \xi } = k \dot { x } + \dot { y } } \\
{ \dot { \eta } = k \dot { x } - \dot { y } }
\end{array} \Leftrightarrow \left\{\begin{array}{l}
\dot{x}=\frac{\dot{\xi}+\dot{\eta}}{2 k} \\
\dot{y}=\frac{\dot{\xi}-\dot{\eta}}{2}
\end{array} .\right.\right.
$$

In this case we have:

If $\left(\dot{\xi}_{n}, \dot{\eta}_{n}\right) \in \mathcal{Z}_{0}, \quad$ that is if $\left\{\begin{array}{l}\dot{\xi}_{n} \leq 0 \\ \dot{\eta}_{n} \leq 0\end{array}\right.$, then $\left\{\begin{array}{l}\dot{\xi}_{n+1}=\dot{\xi}_{n} \\ \dot{\eta}_{n+1}=\dot{\eta}_{n}\end{array}\right.$

If $\left(\dot{\xi}_{n}, \dot{\eta}_{n}\right) \in \mathcal{Z}_{1}$, that is if $\left\{\begin{array}{l}\dot{\xi}_{n} \leq 0 \\ \dot{\eta}_{n}>0\end{array}\right.$, then $\left\{\begin{array}{l}\dot{\xi}_{n+1}=\dot{\xi}_{n}+2 \frac{1-k^{2}}{1+k^{2}} \dot{\eta}_{n} \\ \dot{\eta}_{n+1}=-\dot{\eta}_{n}\end{array}\right.$

If $\left(\dot{\xi}_{n}, \dot{\eta}_{n}\right) \in \mathcal{Z}_{2}$, that is if $\left\{\begin{array}{l}\dot{\xi}_{n}>0 \\ \dot{\eta}_{n} \leq 0\end{array}\right.$, then $\left\{\begin{array}{l}\dot{\xi}_{n+1}=-\dot{\xi}_{n} \\ \dot{\eta}_{n+1}=\dot{\eta}_{n}+2 \frac{1-k^{2}}{1+k^{2}} \dot{\xi}_{n}\end{array}\right.$ 
If $\left(\dot{\xi}_{n}, \dot{\eta}_{n}\right) \in \mathcal{Z}_{12}, \quad$ that is if $\left\{\begin{array}{l}\dot{\xi}_{n}>0 \\ \dot{\eta}_{n}>0\end{array}\right.$, then

$$
\left\{\begin{aligned}
\dot{\xi}_{n+1}= & -\frac{\left(1+k^{2}\right)\left(\dot{\xi}_{n}^{2}+\dot{\eta}_{n}^{2}\right)+2\left(1-k^{2}\right) \dot{\xi}_{n} \dot{\eta}_{n}}{\left(1+k^{2}\right)\left(\dot{\xi}_{n}^{2}+\dot{\eta}_{n}^{2}\right)-2\left(1-k^{2}\right) \dot{\xi}_{n} \dot{\eta}_{n}} \dot{\xi}_{n} \\
& +2 \frac{\left(1-k^{2}\right)\left(\dot{\xi}_{n}^{2}+\dot{\eta}_{n}^{2}\right)}{\left(1+k^{2}\right)\left(\dot{\xi}_{n}^{2}+\dot{\eta}_{n}^{2}\right)-2\left(1-k^{2}\right) \dot{\xi}_{n} \dot{\eta}_{n}} \dot{\eta}_{n} \\
\dot{\eta}_{n+1}= & 2 \frac{\left(1-k^{2}\right)\left(\dot{\xi}_{n}^{2}+\dot{\eta}_{n}^{2}\right)}{\left(1+k^{2}\right)\left(\dot{\xi}_{n}^{2}+\dot{\eta}_{n}^{2}\right)-2\left(1-k^{2}\right) \dot{\xi}_{n} \dot{\eta}_{n}} \dot{\xi}_{n} \\
& -\frac{\left(1+k^{2}\right)\left(\dot{\xi}_{n}^{2}+\dot{\eta}_{n}^{2}\right)+2\left(1-k^{2}\right) \dot{\xi}_{n} \dot{\eta}_{n}}{\left(1+k^{2}\right)\left(\dot{\xi}_{n}^{2}+\dot{\eta}_{n}^{2}\right)-2\left(1-k^{2}\right) \dot{\xi}_{n} \dot{\eta}_{n}} \dot{\eta}_{n}
\end{aligned}\right.
$$

\section{Theoretical results about the ideal impact}

Several results and some remarks can be listed about $\mathrm{TA}_{i d}$. Some of them can be straightforwardly obtained by one or more of the expressions of the algorithm, some others requires a detailed proof.

RESUlt 1. If $\mathbf{v}_{n} \in \mathcal{Z}_{1}$ then $\mathbf{v}_{n+1} \in \mathcal{Z}_{2}$ or $\mathbf{v}_{n+1} \in \mathcal{Z}_{0}$. Analogously, if $\mathbf{v}_{n} \in \mathcal{Z}_{2}$ then $\mathbf{v}_{n+1} \in \mathcal{Z}_{1}$ or $\mathbf{v}_{n+1} \in \mathcal{Z}_{0}$.

Proof: It follows immediately from (7b/7c). If $\mathbf{v}_{n} \in \mathcal{Z}_{1}$ then $\eta_{n}>0$. Then $\eta_{n+1}=-\eta_{n}<0$, so that $\mathbf{v}_{n+1} \in \mathcal{Z}_{2}$ or $\mathbf{v}_{n+1} \in \mathcal{Z}_{0}$. The proof is analogous if $\mathbf{v}_{n} \in \mathcal{Z}_{2}$.

This shows that, if an iteration of $\mathrm{TA}_{i d}$ gives a velocity $\mathbf{v} \notin \mathcal{Z}_{12}$, then all the following velocities do not belong to $\mathcal{Z}_{12}$. In particular, if $\mathbf{v}_{0} \notin \mathcal{Z}_{12}$, than the evolution of the disk will be determined by a sequence of single impacts, without multiple impacts.

ReSult 2. If $\mathbf{v}_{n} \in \mathcal{Z}_{1}$ and $k \geq 1$ then $\mathbf{v}_{n+1} \in \mathcal{Z}_{0}$. Analogously, if $\mathbf{v}_{n} \in \mathcal{Z}_{2}$ and $k \geq 1$ then $\mathbf{v}_{n+1} \in \mathcal{Z}_{0}$.

Proof: It follows once again from (7b/7c). If $\mathbf{v}_{n} \in \mathcal{Z}_{1}$ then $\xi_{n} \leq 0$ and $\eta_{n}>0$. Therefore, if $k \geq 1$, we have $\xi_{n+1}=\xi_{n}+2 \frac{1-k^{2}}{1+k^{2}} \eta_{n}<0$, so that $\mathbf{v}_{n+1} \in \mathcal{Z}_{0}$. The proof is analogous if $\mathbf{v}_{n} \in \mathcal{Z}_{2}$.

This shows that if the angle $2 \alpha \geq \frac{\pi}{2}$ and the impact is not multiple we have only one iteration of $\mathrm{TA}_{i d}$. This is the case, for instance, when $2 \alpha \geq \frac{\pi}{2}$, the disk moves along one of the wall and impacts the other wall.

Result 3. If $\mathbf{v}_{n} \in \mathcal{Z}_{1}$ then there exists $\chi \in \mathbb{N}$ such that $\mathbf{v}_{n+\chi} \in \mathcal{Z}_{0}$. Analogously, if $\mathbf{v}_{n} \in \mathcal{Z}_{2}$ then there exists $\chi \in \mathbb{N}$ such that $\mathbf{v}_{n+\chi} \in \mathcal{Z}_{0}$.

Proof: This is a standard proof about reflections following from (5b $5 \mathrm{c}$ ). If $\mathbf{v}_{n} \in \mathcal{Z}_{1}$ or $\mathcal{Z}_{2}$ and $k \geq 1$ the thesis follows from the point 2) above. 
If $\mathbf{v}_{n} \in \mathcal{Z}_{1}$ and $k<1$, then $\alpha \in\left(0, \frac{\pi}{4}\right)$ and $\varphi_{n} \in(-\pi+\alpha,-\alpha]$. We can construct the odd and even subsequences of the sequence $\varphi_{n+r}$ with $r \in \mathbb{N}$. We have that:

$$
\left\{\begin{array}{l}
\varphi_{n+2 r}=\varphi_{n}-2(2 r) \alpha \\
\varphi_{n+2 r+1}=-\varphi_{n}+2(2 r+1) \alpha
\end{array}\right.
$$

Then $\chi$ is the first natural number such that $\varphi_{n}-2(2 \chi) \alpha \in(-\pi,-\pi+\alpha] \cup$ $[\pi-\alpha, \pi]$ or $-\varphi_{n}+2(2 \chi+1) \alpha \in(-\pi,-\pi+\alpha] \cup[\pi, \pi-\alpha]$. The proof is analogous if $\mathbf{v}_{n} \in \mathcal{Z}_{2}$.

This shows that, if an iteration of $\mathrm{TA}_{i d}$ gives a velocity $\mathbf{v} \notin \mathcal{Z}_{12}$, then $\mathrm{TA}_{i d}$ terminates, giving a final exit velocity for the disk. Note moreover that the reflection procedure of this situation is conceptually identical to the well known one governing the (alternated) single impacts of a disk with the walls of a corner in a sequence of configurations of single (and not multiple) contacts between disk and walls.

The three results above pertain $\mathrm{TA}_{i d}$ applied in the case of single impact of the disk in the corner. However the most significant results are those about multiple impacts. Note that the condition $\mathbf{v}_{n} \in \mathcal{Z}_{12}$ implies that $\dot{x}_{n}>0$ and $\cos \varphi_{n}>0$. We have that:

RESULT 4. If $\mathbf{v}_{n} \in \mathcal{Z}_{12}$ has the direction of the angle bisector, then $\mathbf{v}_{n+1}=-\mathbf{v}_{n} \in \mathcal{Z}_{0}$.

Proof: It follows immediately from (2d) requiring $\dot{y}_{n}=0$ or alternatively from (5d) requiring $\cos \varphi_{n}=1, \sin \varphi_{n}=0$.

The main result about multiple impacts is however the following:

Theorem 3.1 If $\mathbf{v}_{n} \in \mathcal{Z}_{12}$ then $\mathbf{v}_{n+1} \notin \mathcal{Z}_{12}$.

Proof: Let $\mathbf{v}_{n} \in \mathcal{Z}_{12}$ and let us consider (7d). Then $\dot{\xi}_{n}>0, \dot{\eta}_{n}>0$. If $\dot{\xi}_{n}=\dot{\eta}_{n}$ then $\mathbf{v}_{n}$ has the direction of the angle bisector and we have the thesis. Otherwise note that

$$
\left(1+k^{2}\right)\left(\dot{\xi}_{n}^{2}+\dot{\eta}_{n}^{2}\right)-2\left(1-k^{2}\right) \dot{\xi}_{n} \dot{\eta}_{n}=\left(\dot{\xi}_{n}-\dot{\eta}_{n}\right)^{2}+k^{2}\left(\dot{\xi}_{n}+\dot{\eta}_{n}\right)^{2}>0
$$

Defining for brevity $\rho_{n}=\frac{2 \xi_{n} \eta_{n}}{\dot{\xi}_{n}^{2}+\dot{\eta}_{n}^{2}}, \rho_{n} \in(0,1)$, we have then

$$
\left(1+k^{2}\right)-\rho_{n}\left(1-k^{2}\right)>0
$$

and (13) can be rewritten as

$$
\left\{\begin{array}{l}
\dot{\xi}_{n+1}=-\frac{\left(1+k^{2}\right)+\rho_{n}\left(1-k^{2}\right)}{\left(1+k^{2}\right)-\rho_{n}\left(1-k^{2}\right)} \dot{\xi}_{n}+2 \frac{\left(1-k^{2}\right)}{\left(1+k^{2}\right)-\rho_{n}\left(1-k^{2}\right)} \dot{\eta}_{n} \\
\dot{\eta}_{n+1}=2 \frac{\left(1-k^{2}\right)}{\left(1+k^{2}\right)-\rho_{n}\left(1-k^{2}\right)} \dot{\xi}_{n}-\frac{\left(1+k^{2}\right)+\rho_{n}\left(1-k^{2}\right)}{\left(1+k^{2}\right)-\rho_{n}\left(1-k^{2}\right)} \dot{\eta}_{n}
\end{array}\right.
$$


and so:

$$
\left\{\begin{array}{l}
\dot{\xi}_{n+1}=\frac{\dot{\xi}_{n}}{\left(1+k^{2}\right)-\rho_{n}\left(1-k^{2}\right)}\left(-\left(1+k^{2}\right)-\rho_{n}\left(1-k^{2}\right)+2 \frac{\dot{\eta}_{n}}{\dot{\xi}_{n}}\left(1-k^{2}\right)\right) \\
\dot{\eta}_{n+1}=\frac{\dot{\eta}_{n}}{\left(1+k^{2}\right)-\rho_{n}\left(1-k^{2}\right)}\left(-\left(1+k^{2}\right)-\rho_{n}\left(1-k^{2}\right)+2 \frac{\dot{\xi}_{n}}{\dot{\eta}_{n}}\left(1-k^{2}\right)\right)
\end{array}\right.
$$

Suppose by contradiction that $\mathbf{v}_{n+1} \in \mathcal{Z}_{12}$ : then we must have $\dot{\xi}_{n+1}>$ $0, \dot{\eta}_{n+1}>0$, that is

$$
\left\{\begin{array}{l}
\left(2 \frac{\dot{\eta}_{n}}{\dot{\xi}_{n}}-\rho_{n}\right)\left(1-k^{2}\right)>\left(1+k^{2}\right) \\
\left(2 \frac{\dot{\xi}_{n}}{\dot{\eta}_{n}}-\rho_{n}\right)\left(1-k^{2}\right)>\left(1+k^{2}\right)
\end{array}\right.
$$

and recalling the expression of $\rho_{n}$,

$$
\left\{\begin{array}{l}
2\left(1-k^{2}\right) \frac{\dot{\eta}_{n}}{\dot{\xi}_{n}} \frac{\dot{\eta}_{n}^{2}}{\dot{\xi}_{n}^{2}+\dot{\eta}_{n}^{2}}>\left(1+k^{2}\right) \\
2\left(1-k^{2}\right) \frac{\dot{\xi}_{n}}{\dot{\eta}_{n}} \frac{\dot{\xi}_{n}^{2}}{\dot{\xi}_{n}^{2}+\dot{\eta}_{n}^{2}}>\left(1+k^{2}\right)
\end{array}\right.
$$

This is possible only if $1-k^{2}>0$, so that let $k \in(0,1)$. The function $f(k)=$ $\frac{1+k^{2}}{2\left(1-k^{2}\right)}>\frac{1}{2}$ if $k \in(0,1)$. Then a necessary condition for $\mathbf{v}_{n+1} \in \mathcal{Z}_{12}$ is:

$$
\left\{\begin{array}{l}
\frac{\dot{\eta}_{n}}{\dot{\xi}_{n}} \frac{1}{\frac{\dot{\xi}_{n}^{2}+\dot{\eta}_{n}^{2}}{\dot{\eta}_{n}^{2}}}>\frac{1}{2} \\
\frac{\dot{\xi}_{n}}{\dot{\eta}_{n}} \frac{1}{\frac{\dot{\xi}_{n}^{2}+\dot{\eta}_{n}^{2}}{\dot{\xi}_{n}^{2}}}>\frac{1}{2}
\end{array}\right.
$$

Let now for simplicity be $\frac{\dot{\eta}_{n}}{\dot{\xi}_{n}}=z>0$. A straightforward calculation shows that (18) is equivalent to:

$$
\left\{\begin{array} { l } 
{ z \frac { 1 } { \frac { 1 } { z ^ { 2 } } + 1 } > \frac { 1 } { 2 } } \\
{ \frac { 1 } { z } \frac { 1 } { 1 + z ^ { 2 } } > \frac { 1 } { 2 } }
\end{array} \Leftrightarrow \left\{\begin{array} { l } 
{ 2 z ^ { 3 } - z ^ { 2 } - 1 > 0 } \\
{ z ^ { 3 } + z - 2 < 0 }
\end{array} \Leftrightarrow \left\{\begin{array}{l}
z>1 \\
z<1
\end{array} .\right.\right.\right.
$$


This is not possible, and then $\mathbf{v}_{n+1} \notin \mathcal{Z}_{12}$.

We have then the following:

Corollary 3.1 For every initial velocity $\mathbf{v}_{0}$ of the disk, the algorithm terminates after a finite number of steps.

Proof: If $\mathbf{v}_{0} \in \mathcal{Z}_{0}$, there is nothing to proof. If $\mathbf{v}_{0} \in \mathcal{Z}_{1}$ or $\mathbf{v}_{0} \in \mathcal{Z}_{2}$, the thesis follows from Result 3 of this section. If $\mathbf{v}_{0} \in \mathcal{Z}_{12}$, then $\mathbf{v}_{1} \notin \mathcal{Z}_{12}$ and then we have the thesis.

RESUlt 5. Theorem 3.1 and Result 1 imply that the system can have at most one multiple impact if and only if $\mathbf{v}_{0} \in \mathcal{Z}_{12}$.

\section{Theoretical algorithm for the non-ideal case ( $\left.\mathbf{T A}_{n i d}\right)$}

In this section we present two different forms of the iterative rule assigning the "new" velocity $\mathbf{v}_{n+1}$ of the disk as function of the "old" velocity $\mathbf{v}_{n}$ in the non-ideal case. The rule is derived by the theoretical characterization of non-ideal impact presented in Appendix. Each one of the forms will be used to obtain theoretical results about $\mathrm{TA}_{\text {nid }}$.

\subsection{First expression of TA $\mathrm{TA}_{n i d}$ : use of $(\dot{x}, \dot{y})$}

Given an initial velocity $\mathbf{v}_{0}=\left(\dot{x}_{0}, \dot{y}_{0}\right)$, the iterative rule is such that:

If $\left(\dot{x}_{n}, \dot{y}_{n}\right) \in \mathcal{Z}_{0}$, that is if $\left\{\begin{array}{l}k \dot{x}_{n}+\dot{y}_{n} \leq 0 \\ k \dot{x}_{n}-\dot{y}_{n} \leq 0\end{array}\right.$, then $\left\{\begin{array}{l}\dot{x}_{n+1}=\dot{x}_{n} \\ \dot{y}_{n+1}=\dot{y}_{n}\end{array}\right.$

If $\left(\dot{x}_{n}, \dot{y}_{n}\right) \in \mathcal{Z}_{1}$, that is if $\left\{\begin{array}{l}k \dot{x}_{n}+\dot{y}_{n} \leq 0 \\ k \dot{x}_{n}-\dot{y}_{n}>0\end{array}\right.$, then $\left\{\begin{array}{l}\dot{x}_{n+1}=\frac{1-\varepsilon k^{2}}{1+k^{2}} \dot{x}_{n}+\frac{(1+\varepsilon) k}{1+k^{2}} \dot{y}_{n} \\ \dot{y}_{n+1}=\frac{(1+\varepsilon) k}{1+k^{2}} \dot{x}_{n}-\frac{\varepsilon-k^{2}}{1+k^{2}} \dot{y}_{n}\end{array}\right.$

If $\left(\dot{x}_{n}, \dot{y}_{n}\right) \in \mathcal{Z}_{2}$, that is if $\left\{\begin{array}{l}k \dot{x}_{n}+\dot{y}_{n}>0 \\ k \dot{x}_{n}-\dot{y}_{n} \leq 0\end{array}\right.$, then $\left\{\begin{array}{l}\dot{x}_{n+1}=\frac{1-\varepsilon k^{2}}{1+k^{2}} \dot{x}_{n}-\frac{(1+\varepsilon) k}{1+k^{2}} \dot{y}_{n} \\ \dot{y}_{n+1}=-\frac{(1+\varepsilon) k}{1+k^{2}} \dot{x}_{n}-\frac{\varepsilon-k^{2}}{1+k^{2}} \dot{y}_{n}\end{array}\right.$

If $\left(\dot{x}_{n}, \dot{y}_{n}\right) \in \mathcal{Z}_{12}$, that is if $\left\{\begin{array}{l}k \dot{x}_{n}+\dot{y}_{n}>0 \\ k \dot{x}_{n}-\dot{y}_{n}>0\end{array}\right.$, then $\left\{\begin{array}{l}\dot{x}_{n+1}=\frac{-\varepsilon k^{4} \dot{x}_{n}^{2}+\left(1-(1+\varepsilon) k^{2}\right) \dot{y}_{n}^{2}}{k^{4} \dot{x}_{n}^{2}+\dot{y}_{n}^{2}} \dot{x}_{n} \\ \dot{y}_{n+1}=\frac{k^{2}\left(k^{2}-(1+\varepsilon)\right) \dot{x}_{n}^{2}-\varepsilon \dot{y}_{n}^{2}}{k^{4} \dot{x}_{n}^{2}+\dot{y}_{n}^{2}} \dot{y}_{n}\end{array}\right.$ 
REMARK 4. The iterative rule (14a 14d once again respects the symmetry of the mechanical problem with respect to the $(\dot{x}, \dot{y})$ components of the velocity, since relations (4) hold for every $\mathbf{v}_{n} \notin \mathcal{Z}_{0}$.

\subsection{Second expression of $\mathrm{TA}_{\text {nid }}$ : use of $(\dot{\xi}, \dot{\eta})$}

The algorithm can be expressed once again by using the coordinates $(\xi, \eta)=$ $(k x+y, k x-y)$. We obtain:

If $\left(\dot{\xi}_{n}, \dot{\eta}_{n}\right) \in \mathcal{Z}_{0}, \quad$ that is if $\left\{\begin{array}{l}\dot{\xi}_{n} \leq 0 \\ \dot{\eta}_{n} \leq 0\end{array}\right.$, then $\left\{\begin{array}{l}\dot{\xi}_{n+1}=\dot{\xi}_{n} \\ \dot{\eta}_{n+1}=\dot{\eta}_{n}\end{array}\right.$

If $\left(\dot{\xi}_{n}, \dot{\eta}_{n}\right) \in \mathcal{Z}_{1}$, that is if $\left\{\begin{array}{l}\dot{\xi}_{n} \leq 0 \\ \dot{\eta}_{n}>0\end{array}\right.$, then $\left\{\begin{array}{l}\dot{\xi}_{n+1}=\dot{\xi}_{n}+(1+\varepsilon) \frac{1-k^{2}}{1+k^{2}} \dot{\eta}_{n} \\ \dot{\eta}_{n+1}=-\varepsilon \dot{\eta}_{n}\end{array}\right.$

If $\left(\dot{\xi}_{n}, \dot{\eta}_{n}\right) \in \mathcal{Z}_{2}, \quad$ that is if $\left\{\begin{array}{l}\dot{\xi}_{n}>0 \\ \dot{\eta}_{n} \leq 0\end{array}\right.$, then $\left\{\begin{array}{l}\dot{\xi}_{n+1}=-\varepsilon \dot{\xi}_{n} \\ \dot{\eta}_{n+1}=\dot{\eta}_{n}+(1+\varepsilon) \frac{1-k^{2}}{1+k^{2}} \dot{\xi}_{n}\end{array}\right.$

If $\left(\dot{\xi}_{n}, \dot{\eta}_{n}\right) \in \mathcal{Z}_{12}$, that is if $\left\{\begin{array}{l}\dot{\xi}_{n}>0 \\ \dot{\eta}_{n}>0\end{array}\right.$, then

$$
\left\{\begin{aligned}
\dot{\xi}_{n+1}= & -\frac{\varepsilon\left(1+k^{2}\right)\left(\dot{\xi}_{n}^{2}+\dot{\eta}_{n}^{2}\right)+2\left(1-k^{2}\right) \dot{\xi}_{n} \dot{\eta}_{n}}{\left(1+k^{2}\right)\left(\dot{\xi}_{n}^{2}+\dot{\eta}_{n}^{2}\right)-2\left(1-k^{2}\right) \dot{\xi}_{n} \dot{\eta}_{n}} \dot{\xi}_{n} \\
& +(1+\varepsilon) \frac{\left(1-k^{2}\right)\left(\dot{\xi}_{n}^{2}+\dot{\eta}_{n}^{2}\right)}{\left(1+k^{2}\right)\left(\dot{\xi}_{n}^{2}+\dot{\eta}_{n}^{2}\right)-2\left(1-k^{2}\right) \dot{\xi}_{n} \dot{\eta}_{n}} \dot{\eta}_{n} \\
\dot{\eta}_{n+1}= & (1+\varepsilon) \frac{\left(1-k^{2}\right)\left(\dot{\xi}_{n}^{2}+\dot{\eta}_{n}^{2}\right)}{\left(1+k^{2}\right)\left(\dot{\xi}_{n}^{2}+\dot{\eta}_{n}^{2}\right)-2\left(1-k^{2}\right) \dot{\xi}_{n} \dot{\eta}_{n}} \dot{\xi}_{n} \\
& -\frac{\varepsilon\left(1+k^{2}\right)\left(\dot{\xi}_{n}^{2}+\dot{\eta}_{n}^{2}\right)+2\left(1-k^{2}\right) \dot{\xi}_{n} \dot{\eta}_{n}}{\left(1+k^{2}\right)\left(\dot{\xi}_{n}^{2}+\dot{\eta}_{n}^{2}\right)-2\left(1-k^{2}\right) \dot{\xi}_{n} \dot{\eta}_{n}} \dot{\eta}_{n}
\end{aligned}\right.
$$

REMARK 5. Note that, due to the change of coordinates (6), we have:

$$
\left(\left\|\mathbf{v}_{n}\right\|_{2}\right)^{2}=\frac{1+k^{2}}{4 k^{2}}\left(\dot{\xi}_{n}^{2}+\dot{\eta}_{n}^{2}\right)+\frac{1-k^{2}}{2 k^{2}} \dot{\xi}_{n} \dot{\eta}_{n}
$$




\section{Theoretical results about the non-ideal impact}

Several results and some remarks that can be listed about $\mathrm{TA}_{n i d}$ are strictly analogous to those about $\mathrm{TA}_{i d}$. For instance, Results 1,2 and 4 can be immediately generalized to the non-ideal case, with proofs and remarks analogous to those presented in Sec.3. Theorem 3.1 too holds in the nonideal case, as we prove below in this section. Instead, in the non-ideal case we cannot state the analogous of Result 3 of Sec.3, that in the ideal case is crucial to prove that $\mathrm{TA}_{i d}$ terminates. However, for $\mathrm{TA}_{\text {nid }}$, the termination will be ensured on the basis of the criterion $\lim _{n \rightarrow+\infty}\left\|\mathbf{v}_{n}\right\|_{2}=0$ of the following theorem (5.3).

Theorem 5.1 If $\mathbf{v}_{n} \in \mathcal{Z}_{12}$ then $\mathbf{v}_{n+1} \notin \mathcal{Z}_{12}$.

Proof: Let $\mathbf{v}_{n}$ be in $\mathcal{Z}_{12}$, so that $\dot{\xi}_{n}>0$ and $\dot{\eta}_{n}>0$. Recalling that $\varepsilon \in[0,1)$ and $k \in(0,+\infty)$, we set

$$
\beta=\frac{1-k^{2}}{1+k^{2}} \in(-1,1), \quad z=\frac{\dot{\eta}_{n}}{\dot{\xi}_{n}} \in(0,+\infty) .
$$

We have $\frac{2 \dot{\xi}_{n} \dot{\eta}_{n}}{\dot{\xi}_{n}^{2}+\dot{\eta}_{n}^{2}}=\frac{2 z}{1+z^{2}}$ and eqs. (15d) can be rewritten as

$$
\left\{\begin{array}{l}
\dot{\xi}_{n+1}=\frac{\dot{\xi}_{n}}{1-\beta \frac{2 z}{1+z^{2}}}\left(-\varepsilon+\beta\left((1+\varepsilon) z-\frac{2 z}{1+z^{2}}\right)\right) \\
\dot{\eta}_{n+1}=\frac{\dot{\eta}_{n}}{1-\beta \frac{2 z}{1+z^{2}}}\left(-\varepsilon+\beta\left((1+\varepsilon) \frac{1}{z}-\frac{2 z}{1+z^{2}}\right)\right)
\end{array}\right.
$$

where the two first factors of the RHSs are positive. Then $\mathbf{v}_{n+1} \notin \mathcal{Z}_{12}$ if and only if the system of inequalities

$$
\left\{\begin{array}{l}
-\varepsilon+\beta\left((1+\varepsilon) z-\frac{2 z}{1+z^{2}}\right)>0 \\
-\varepsilon+\beta\left((1+\varepsilon) \frac{1}{z}-\frac{2 z}{1+z^{2}}\right)>0
\end{array}\right.
$$

does not admit solutions for $\varepsilon \in[0,1), \beta \in(-1,1), z \in(0,+\infty)$. Obviously (16) does not have solutions if $\beta=0$ (that is when the amplitude $2 \alpha$ of the corner is $\frac{\pi}{2}$ ), if $z=1$ (that is when $\mathbf{v}_{n}$ is along the bisector of the corner), if $(1+\varepsilon) z-\frac{2 z}{1+z^{2}}=0$, if $(1+\varepsilon) \frac{1}{z}-\frac{2 z}{1+z^{2}}=0$. 
If $\varepsilon=0, \beta \in(0,1)$, then (16) is equivalent to

$$
\left\{\begin{array} { l } 
{ z - \frac { 2 z } { 1 + z ^ { 2 } } > 0 } \\
{ \frac { 1 } { z } - \frac { 2 z } { 1 + z ^ { 2 } } > 0 }
\end{array} \quad \Rightarrow \quad \left\{\begin{array}{l}
z^{2}-1>0 \\
1-z^{2}>0
\end{array}\right.\right.
$$

that does not have solutions $\forall z \in(0,+\infty)$. If $\varepsilon=0, \beta \in(-1,0)$, then (16) is equivalent to

$$
\left\{\begin{array} { l } 
{ z - \frac { 2 z } { 1 + z ^ { 2 } } < 0 } \\
{ \frac { 1 } { z } - \frac { 2 z } { 1 + z ^ { 2 } } < 0 }
\end{array} \quad \Rightarrow \quad \left\{\begin{array}{l}
z^{2}-1<0 \\
1-z^{2}<0
\end{array}\right.\right.
$$

that does not have solutions $\forall z \in(0,+\infty)$. If $\varepsilon \in(0,1), \beta \in(-1,0)$, then (16) implies that

$$
\left\{\begin{array} { l } 
{ ( 1 + \varepsilon ) z - \frac { 2 z } { 1 + z ^ { 2 } } < 0 } \\
{ ( 1 + \varepsilon ) \frac { 1 } { z } - \frac { 2 z } { 1 + z ^ { 2 } } < 0 }
\end{array} \Rightarrow \left\{\begin{array}{l}
\varepsilon<\frac{1-z^{2}}{1+z^{2}} \\
\varepsilon<-\frac{1-z^{2}}{1+z^{2}},
\end{array}\right.\right.
$$

that does not have solutions $\forall z \in(0,+\infty)$.

If $\varepsilon \in(0,1), \beta \in(0,1), z \in(0,1)$, then the first inequality of (16) can be verified only if $(1+\varepsilon) z-\frac{2 z}{1+z^{2}}>0$. In this case we have:

$$
\beta>\frac{\varepsilon}{(1+\varepsilon) z-\frac{2 z}{1+z^{2}}}=\frac{\varepsilon\left(1+z^{2}\right)}{(1+\varepsilon)\left(z+z^{3}\right)-2 z}
$$

This can happen only if

$$
\frac{\varepsilon\left(1+z^{2}\right)}{(1+\varepsilon)\left(z+z^{3}\right)-2 z}<1 \quad \Leftrightarrow \quad \varepsilon<-\frac{z(1+z)}{1+z^{2}}<0
$$

that is not admissible. If $\varepsilon \in(0,1), \beta \in(0,1), z \in(1,+\infty)$, then the second inequality of (16) can be verified only if $(1+\varepsilon) \frac{1}{z}-\frac{2 z}{1+z^{2}}>0$. In this case we have:

$$
\beta>\frac{\varepsilon}{(1+\varepsilon) \frac{1}{z}-\frac{2 z}{1+z^{2}}}=\frac{\varepsilon z\left(1+z^{2}\right)}{(1+\varepsilon)\left(1+z^{2}\right)-2 z^{2}}
$$

This can happen only if

$$
\frac{\varepsilon z\left(1+z^{2}\right)}{(1+\varepsilon)\left(1+z^{2}\right)-2 z^{2}}<1 \quad \Leftrightarrow \quad \varepsilon<-\frac{1+z}{1+z^{2}}<0
$$


that is not admissible. It follows that (16) cannot have solutions, and then $\mathbf{v}_{n+1} \notin \mathcal{Z}_{12}$.

RESUlT 6. Theorem 5.1 and Result 1 (that holds for non-ideal impacts too) imply once again that the system can have at most one multiple impact if and only if $\mathbf{v}_{0} \in \mathcal{Z}_{12}$.

To proof the second important result about $\mathrm{TA}_{\text {nid }}$ we need to introduce the convergent matrices and their properties. Let $A$ be an $N \times N$ matrix and let $\rho(A)$ its spectral radius, that is the largest modulus of its eigenvalues. We recall that the matrix $A$ is said to be convergent if $\lim _{k \rightarrow+\infty}\left(A^{k}\right)_{i j}=0$ for each $i, j=1, \ldots, N$, where $\left(A^{k}\right)_{i j}$ is the $(i, j)$-th element of $A^{k}$. The following theorem (see e.g. Theorem 4 in [8, p. 14]) shows some well-known properties of a convergent matrix.

Theorem 5.2 The following three statements are equivalent:

1. the matrix $A$ is convergent;

2. $\lim _{k \rightarrow+\infty}\left\|A^{k}\right\|=0$ for some matrix norm induced by a vector norm, that is defined by $\|A\|=\max _{\|x\|=1}\|A x\|$;

3. $\rho(A)<1$.

Remark 6. Let $A$ be a convergent matrix and let $\|\cdot\|$ be the induced matrix norm for which item 2 of Theorem 5.2 holds. Given a vector w, we have, from a property of the induced matrix norm, that $0 \leq\left\|A^{k} \mathbf{w}\right\| \leq$ $\left\|A^{k}\right\|\|\mathbf{w}\|$ and so $\lim _{k \rightarrow+\infty}\left\|A^{k} \mathbf{w}\right\|=0$. It follows that the vector $A^{k} \mathbf{w}$ converges to the zero vector.

Theorem 5.3 If $\mathbf{v}_{n} \notin \mathcal{Z}_{0}$ for every $n$, then $\lim _{n \rightarrow+\infty}\left\|\mathbf{v}_{n}\right\|=0$.

Proof: Result 6 implies that $\mathbf{v}_{1} \notin \mathcal{Z}_{12}$. Let once again be $\beta=\left(1-k^{2}\right) /\left(1+k^{2}\right)$. By hypothesis, due to Result 6 , we can take $k \in(0,1)$ and then $\beta \in(0,1)$.

Let us suppose that $\mathbf{v}_{1}=\left(\dot{\xi}_{1}, \dot{\eta}_{1}\right) \in \mathcal{Z}_{1}$. Result 5 and the hypothesis imply that $\mathbf{v}_{3}=\left(\dot{\xi}_{3}, \dot{\eta}_{3}\right) \in \mathcal{Z}_{1}$. Applying (15b]15c) we have

$$
\left(\begin{array}{l}
\dot{\xi}_{3} \\
\dot{\eta}_{3}
\end{array}\right)=\left(\begin{array}{cc}
-\varepsilon & -\beta \varepsilon(1+\varepsilon) \\
\beta(1+\varepsilon) & \beta^{2}(1+\varepsilon)^{2}-\varepsilon
\end{array}\right)\left(\begin{array}{l}
\dot{\xi}_{1} \\
\dot{\eta}_{1}
\end{array}\right)
$$

Therefore, for every $h \in \mathbb{N}$, we have

$$
\left(\begin{array}{l}
\dot{\xi}_{2 h+1} \\
\dot{\eta}_{2 h+1}
\end{array}\right)=\left(\begin{array}{cc}
-\varepsilon & -\beta \varepsilon(1+\varepsilon) \\
\beta(1+\varepsilon) & \beta^{2}(1+\varepsilon)^{2}-\varepsilon
\end{array}\right)^{h}\left(\begin{array}{l}
\dot{\xi}_{1} \\
\dot{\eta}_{1}
\end{array}\right)
$$


Moreover, if $\mathbf{v}_{1} \in \mathcal{Z}_{1}$, by Result 5 and the hypothesis we have that $\mathbf{v}_{2} \in \mathcal{Z}_{2}$. A straigthforward calculation shows that in this case, for every $h \in \mathbb{N}, h>0$, we have

$$
\left(\begin{array}{c}
\dot{\xi}_{2 h} \\
\dot{\eta}_{2 h}
\end{array}\right)=\left(\begin{array}{cc}
\beta^{2}(1+\varepsilon)^{2}-\varepsilon & \beta(1+\varepsilon) \\
-\beta \varepsilon(1+\varepsilon) & -\varepsilon
\end{array}\right)^{h}\left(\begin{array}{c}
\dot{\xi}_{2} \\
\dot{\eta}_{2}
\end{array}\right)
$$

Since the two matrices

$$
H_{1}=\left(\begin{array}{cc}
-\varepsilon & -\beta \varepsilon(1+\varepsilon) \\
\beta(1+\varepsilon) & \beta^{2}(1+\varepsilon)^{2}-\varepsilon
\end{array}\right) \quad H_{2}=\left(\begin{array}{cc}
\beta^{2}(1+\varepsilon)^{2}-\varepsilon & \beta(1+\varepsilon) \\
-\beta \varepsilon(1+\varepsilon) & -\varepsilon
\end{array}\right)
$$

have the same characteristic polynomial and eigenvalues, then Theorem 5.2 and Remark 6 imply that $\lim _{n \rightarrow+\infty}\left(\dot{\xi}_{n}, \dot{\eta}_{n}\right)=(0,0)$ if the spectral radius $\rho\left(H_{1}\right)=\rho\left(H_{2}\right)$ is such that $\rho\left(H_{1}\right)<1$. Therefore the theorem follows upon proof that $\rho\left(H_{1}\right)<1$. Needless to say, the proof is completely analogous if $\mathbf{v}_{1} \in \mathcal{Z}_{2}$.

The characteristic polynomial of $H_{1}$ is

$$
p_{H_{1}}(\lambda)=\lambda^{2}-\left(\beta^{2}(1+\varepsilon)^{2}-2 \varepsilon\right) \lambda+\varepsilon^{2},
$$

whose corresponding eigenvalues are

$$
\lambda_{12}=\frac{1}{2}\left(\beta^{2}(1+\varepsilon)^{2}-2 \varepsilon \pm \beta(1+\varepsilon) \sqrt{\beta^{2}(1+\varepsilon)^{2}-4 \varepsilon}\right)
$$

where we have $\lambda_{1} \lambda_{2}=\varepsilon^{2}$.

If $\beta^{2}(1+\varepsilon)^{2}-4 \varepsilon<0$, the eigenvalues are complex conjugates with the same module and then $\rho\left(H_{1}\right)=\left|\lambda_{1}\right|=\left|\lambda_{2}\right|=\varepsilon<1$.

If $\beta^{2}(1+\varepsilon)^{2}-4 \varepsilon=0$, then $\lambda_{1}=\lambda_{2}=\varepsilon<1$.

If $\beta^{2}(1+\varepsilon)^{2}-4 \varepsilon>0$, the eigenvalues are both in $\mathbb{R}$ and they have the same sign. In particular, since $\beta^{2}(1+\varepsilon)^{2}-2 \varepsilon>0$, then $\rho\left(H_{1}\right)=$ $\max \left\{\left|\lambda_{1}\right|,\left|\lambda_{2}\right|\right\}=\frac{1}{2}\left(\beta^{2}(1+\varepsilon)^{2}-2 \varepsilon+\beta(1+\varepsilon) \sqrt{\beta^{2}(1+\varepsilon)^{2}-4 \varepsilon}\right)$.

A standard study of

$$
\rho\left(H_{1}\right)(\varepsilon, \beta)=\frac{1}{2}\left(\beta^{2}(1+\varepsilon)^{2}-2 \varepsilon+\beta(1+\varepsilon) \sqrt{\beta^{2}(1+\varepsilon)^{2}-4 \varepsilon}\right)
$$

in the compact set $\bar{\Theta}=\left\{(\varepsilon, \beta) \mid \varepsilon \in[0,1], \beta \in\left[\frac{2 \sqrt{\varepsilon}}{1+\varepsilon}, 1\right]\right\}$ shows that, since $\frac{\partial \rho\left(H_{1}\right)}{\partial \beta}>0$, the maximum is taken in the segment $\{\beta=1\}$ and $\max _{\bar{\Theta}}\left(\rho\left(H_{1}\right)\right)=$ 1. Then for every fixed $(\varepsilon, \beta) \in \Theta=\left\{(\varepsilon, \beta) \mid \varepsilon \in(0,1), \beta \in\left(\frac{2 \sqrt{\varepsilon}}{1+\varepsilon}, 1\right)\right\}$ we have $\max _{\Theta}\left(\rho\left(H_{1}\right)\right)<1$. 
In conclusion, for every $\varepsilon \in(0,1), k \in(0,1)$ we have $\rho\left(H_{1}\right) \in[\varepsilon, 1)$. Since $\lim _{n \rightarrow+\infty}\left\|\left(\dot{\xi}_{n}, \dot{\eta}_{n}\right)\right\|=0=\lim _{n \rightarrow+\infty}\left\|\left(\dot{x}_{n}, \dot{y}_{n}\right)\right\|$ obviously implies that $\lim _{n \rightarrow+\infty}\left\|\mathbf{v}_{n}\right\|_{2}=0$, we have the thesis.

Remark 7. Let be $\mathbf{v}_{0} \in \mathcal{Z}_{12}$ and $\varepsilon \in(0,1)$. A tedious but straightforward calculation 1 shows that

$$
\left\|\mathbf{v}_{1}\right\|_{2}^{2}-\left\|\mathbf{v}_{0}\right\|_{2}^{2}=\left(\varepsilon^{2}-1\right) \frac{\left(k^{2} \dot{x}_{0}^{2}+\dot{y}_{0}^{2}\right)^{2}}{k^{4} \dot{x}_{0}^{2}+\dot{y}_{0}^{2}}<0 \quad \Rightarrow \quad\left\|\mathbf{v}_{1}\right\|_{2}<\left\|\mathbf{v}_{0}\right\|_{2} .
$$

Moreover, another straightforward calculation shows that

$$
\mathbf{v}_{n} \in \mathcal{Z}_{1} \Rightarrow\left\|\mathbf{v}_{n+1}\right\|_{2}<\left\|\mathbf{v}_{n}\right\|_{2}, \quad \mathbf{v}_{n} \in \mathcal{Z}_{2} \Rightarrow\left\|\mathbf{v}_{n+1}\right\|_{2}<\left\|\mathbf{v}_{n}\right\|_{2} \quad \forall n \geq 0
$$

and then the whole sequence $\left(\left\|\mathbf{v}_{n}\right\|_{2}\right)_{n \geq 0}$ decreases to 0 .

REMARK 8. Theorem 5.3 states a physical property of the mechanical system and not only a numerical property of $\mathrm{TA}_{\text {nid }}$. For example, the same procedure of the proof applied starting from the rule (14) instead of (15) leads to the analysis of the spectral radius of the matrices

$$
\begin{gathered}
K_{1}=\left(\begin{array}{cc}
\frac{\left(1-\varepsilon k^{2}\right)^{2}-k^{2}(1+\varepsilon)^{2}}{\left(1+k^{2}\right)^{2}} & \frac{k\left(1-k^{2}\right)(1+\varepsilon)^{2}}{\left(1+k^{2}\right)^{2}} \\
-\frac{k\left(1-k^{2}\right)(1+\varepsilon)^{2}}{\left(1+k^{2}\right)^{2}} & \frac{\left(\varepsilon-k^{2}\right)^{2}-k^{2}(1+\varepsilon)^{2}}{\left(1+k^{2}\right)^{2}}
\end{array}\right) \\
K_{2}=\left(\begin{array}{cc}
\frac{\left(1-\varepsilon k^{2}\right)^{2}-k^{2}(1+\varepsilon)^{2}}{\left(1+k^{2}\right)^{2}} & -\frac{k\left(1-k^{2}\right)(1+\varepsilon)^{2}}{\left(1+k^{2}\right)^{2}} \\
\frac{k\left(1-k^{2}\right)(1+\varepsilon)^{2}}{\left(1+k^{2}\right)^{2}} & \frac{\left(\varepsilon-k^{2}\right)^{2}-k^{2}(1+\varepsilon)^{2}}{\left(1+k^{2}\right)^{2}}
\end{array}\right) .
\end{gathered}
$$

It can be easily shown that the matrix $B=\left(\begin{array}{cc}k & 1 \\ k & -1\end{array}\right)$ that expresses the change of coordinates (6) is such that $K_{1}=B^{-1} H_{1} B, K_{2}=B^{-1} H_{2} B$. The matrices $H_{1}, H_{2}$ and $K_{1}, K_{2}$ are then respectively similar, they have the same eigenvalues and then the same spectral radius. Similar arguments hold for every admissible change of coordinates.

REMARK 9. For known results on matrices (the so called Gelfand's formula. See e.g. Theorem 4 in $\left[9\right.$, p. 28]), the spectral radius $\rho\left(H_{1}\right)$ of the matrix $H_{1}$ can be expressed as a limit of matrix norms, that is

$$
\rho\left(H_{1}\right)=\lim _{h \rightarrow \infty}\left\|H_{1}^{h}\right\|^{\frac{1}{h}} .
$$

\footnotetext{
${ }^{1}$ The calculation was helped by the use of the factorization command of $\mathrm{CoCoa}^{\circledR}$, a freely available program for computing with multivariate polynomials.
} 
It follows that, for a large enough $h$, we have $\left\|H_{1}^{h}\right\| \approx \rho\left(H_{1}\right)^{h}$, and so

$$
\left\|H_{1}^{h} v_{1}\right\| \leq\left\|H_{1}^{h}\right\|\left\|v_{1}\right\| \approx \rho\left(H_{1}\right)^{h}\left\|v_{1}\right\| .
$$

Therefore the spectral radius $\rho\left(H_{1}\right)=\rho\left(K_{1}\right)$ gives also a measure of the rate of convergence to 0 of the velocity $\mathbf{v}_{n}$. It follows then from the proof of Theorem (5.3) that the bigger $\varepsilon$ and $\beta$ are, the slower the convergence is. This means that we can forecast slow convergence to 0 of the velocity for "almost elastic" walls and very small angles $\alpha$.

\section{PART 2: NUMERICAL ASPECTS}

\section{The numerical results}

We already said that data uncertainty due to possible errors in the realworld measurements and algorithmic errors due to the use of floating point arithmetic can cause disastrous effects on the result. The theoretical algorithms TA can be easily implemented, but they present some numerical drawbacks, because of their sensitivity to the noise on the input data and of its instability with respect to the floating point arithmetic: small perturbations of $\dot{\xi}_{n}$ and $\dot{\eta}_{n}$ can cause structural changes in response, e.g. it can happen that the exact $\dot{\xi}_{n}$ is a small negative value while the computed $\dot{\xi}_{n}$ is a small positive value, causing a different choice in the iterative method.

For this reason we present a numerical algorithm obtained by changing the tests for the choice of the iterative step to do. A threshold $S$ is introduced to consider as zero value the very small positive $\dot{\xi}_{n}$ or $\dot{\eta}_{n}$. Analogously, we introduce a test on the norm of the final linear velocity, in order to consider as (almost) at rest a disk whose computed velocity is less than a very small threshold $S_{v}$.

Obviously, if $S=0$ and $S_{v}=0$ the numerical algorithm coincides with the theoretical ones.

\subsection{Numerical Algorithm (NA)}

- Input: the coefficient $\varepsilon \in[0,1]$, the angle $\alpha \in\left(0, \frac{\pi}{4}\right)$, the initial velocity $\mathbf{v}_{0}=\left(\dot{x}_{0}, \dot{y}_{0}\right)$, with $\left\|\mathbf{v}_{0}\right\|_{2}=1$, and the threshold $S$ and $S_{v}$.

- Output: the final velocity $\mathbf{v}_{f}$.

- First step: $k=\tan (\alpha) ; n=0 ; \dot{\xi}_{0}=k \dot{x}_{0}+\dot{y}_{0} ; \dot{\eta}_{0}=k \dot{x}_{0}-\dot{y}_{0}$.

- Core: While $\left(\dot{\xi}_{n}>S\right.$ or $\left.\dot{\eta}_{n}>S\right), n<N_{\max }$, and $\left\|\left(\dot{x}_{n}, \dot{y}_{n}\right)\right\|_{2}>S_{v}$ : 
1. if $\left(\dot{\xi}_{n} \leq S\right)$ and $\left(\dot{\eta}_{n}>S\right)$ then

$$
\left\{\begin{array}{l}
\dot{x}_{n+1}=\frac{1-\varepsilon k^{2}}{1+k^{2}} \dot{x}_{n}+\frac{(1+\varepsilon) k}{1+k^{2}} \dot{y}_{n} \\
\dot{y}_{n+1}=\frac{(1+\varepsilon) k}{1+k^{2}} \dot{x}_{n}-\frac{\varepsilon-k^{2}}{1+k^{2}} \dot{y}_{n}
\end{array}\right.
$$

2. if $\left(\dot{\xi}_{n}>S\right)$ and $\left(\dot{\eta}_{n} \leq S\right)$ then

$$
\left\{\begin{array}{l}
\dot{x}_{n+1}=\frac{1-\varepsilon k^{2}}{1+k^{2}} \dot{x}_{n}-\frac{(1+\varepsilon) k}{1+k^{2}} \dot{y}_{n} \\
\dot{y}_{n+1}=-\frac{(1+\varepsilon) k}{1+k^{2}} \dot{x}_{n}-\frac{\varepsilon-k^{2}}{1+k^{2}} \dot{y}_{n}
\end{array}\right.
$$

3. if $\left(\dot{\xi}_{n}>S\right)$ and $\left(\dot{\eta}_{n}>S\right)$ then

$$
\left\{\begin{array}{l}
\dot{x}_{n+1}=\frac{-\varepsilon k^{4} \dot{x}_{n}^{2}+\left(1-(1+\varepsilon) k^{2}\right) \dot{y}_{n}^{2}}{k^{4} \dot{x}_{n}^{2}+\dot{y}_{n}^{2}} \dot{x}_{n} \\
\dot{y}_{n+1}=\frac{k^{2}\left(k^{2}-(1+\varepsilon)\right) \dot{x}_{n}^{2}-\varepsilon \dot{y}_{n}^{2}}{k^{4} \dot{x}_{n}^{2}+\dot{y}_{n}^{2}} \dot{y}_{n} .
\end{array}\right.
$$

4. $\dot{\xi}_{n+1}=k \dot{x}_{n+1}+\dot{y}_{n+1} ; \dot{\eta}_{n+1}=k \dot{x}_{n+1}-\dot{y}_{n+1} \quad ; n=n+1$.

- $\mathbf{v}_{f}=\left(\dot{x}_{n}, \dot{y}_{n}\right)$.

The previous algorithm stops when both $\dot{\xi}_{n}$ and $\dot{\eta}_{n}$ are less than $S$, or when the 2-norm of the computed velocity is less than $S_{v}$, or when the number of steps exceeds the predefined number $N_{\max }$ of cycles.

The following simple example shows the sensitivity of TA to the noise on the input data and to the floating point arithmetic computation even in a very simple case.

EXAMPLE. We consider the behaviors of TA and NA when they process the initial velocity $\mathbf{v}_{0}=\left(\dot{x}_{0}, \dot{y}_{0}\right)=\left(\frac{1}{\sqrt{2}}, \frac{1}{\sqrt{2}}\right)$, with $\alpha=\frac{\pi}{4}$ and $\varepsilon=1$. From the theoretical point of view, TA and NA process the input data in the same way. Since $\mathbf{v}_{0}$ satisfies the condition of $\mathcal{Z}_{2}$, at the first iteration both algorithms compute the new velocity $\mathbf{v}_{1}=\left(\dot{x}_{1}, \dot{y}_{1}\right)=\left(-\dot{y}_{0},-\dot{x}_{0}\right)=\left(-\frac{1}{\sqrt{2}},-\frac{1}{\sqrt{2}}\right)$. Since the coordinates of $\mathbf{v}_{1}$ satisfies the conditions in $\mathcal{Z}_{0}$ for both algorithms, TA and NA stop and $\mathbf{v}_{1}$ is the final computed velocity.

Nevertheless, when the algorithms are implemented, TA suffers from the data error and the computational approximation, while NA has the same behavior of the theoretical case, in the absence of errors. In fact, since the computed values of $\dot{x}_{1}$ and $\dot{y}_{1}$ are perturbed by errors, we obtain 
$\dot{x}_{1}=-0.707106781186547$ and $\dot{y}_{1}=-0.707106781186548$, so that $\dot{\xi}_{1}=$ -0.707106781186547 and $\dot{\eta}_{1}=4.440892098500626 e-16$. If $S=0$, that is using an implementation of TA, $\dot{\xi}_{1}$ and $\dot{\eta}_{1}$ satisfy the conditions of $\mathcal{Z}_{1}$, and the algorithm compute a new iteration. Differently, NA is more robust and, choosing $S=2 \cdot$ eps, the values $\dot{\xi}_{1}$ and $\dot{\eta}_{1}$ satisfy the conditions of $\mathcal{Z}_{0}$, and the algorithm stops, as in the theoretical case.

\subsection{Comparison between TA and NA}

In the following we show that TA and NA compute the same final velocity in the same number of steps or, even if one of the algorithms executes more iterations, the final velocities are very similar. We can conclude that NA is preferable when we deal with real world measurements, since it produces analogous final velocities as TA, but it is more robust with respect to the errors on the input data.

Lemma 6.1 Let $\left(\dot{x}_{n}, \dot{y}_{n}\right)$ be the linear velocity at the current step. Then:

i) if $\left(\dot{x}_{n}, \dot{y}_{n}\right) \in \mathcal{Z}_{1}$ we have $\quad\left|\dot{x}_{n+1}-\dot{x}_{n}\right| \leq \dot{\eta}_{n} \quad$ and $\quad\left|\dot{y}_{n+1}-\dot{y}_{n}\right| \leq 2 \dot{\eta}_{n}$;

ii) if $\left(\dot{x}_{n}, \dot{y}_{n}\right) \in \mathcal{Z}_{2}$ we have $\left|\dot{x}_{n+1}-\dot{x}_{n}\right| \leq \dot{\xi}_{n} \quad$ and $\quad\left|\dot{y}_{n+1}-\dot{y}_{n}\right| \leq 2 \dot{\xi}_{n}$.

Proof: By direct computation, the relations in $\mathcal{Z}_{1}$ give

$$
\dot{x}_{n+1}-\dot{x}_{n}=-\frac{(1+\varepsilon) k}{1+k^{2}} \dot{\eta}_{n} \quad \text { and } \quad \dot{y}_{n+1}-\dot{y}_{n}=\frac{(1+\varepsilon)}{1+k^{2}} \dot{\eta}_{n}
$$

so that the thesis follows, since $\varepsilon, k<1$ and $\dot{\eta}_{n}>0$. Analogously for $\mathcal{Z}_{2}$, changing the role of $\dot{\xi}_{n}$ and $\dot{\eta}_{n}$.

Lemma 6.2 Let $\mathbf{v}=(\dot{x}, \dot{y})$ and $\mathbf{v}_{\mathbf{p}}=\left(\dot{x}+\delta_{x}, \dot{y}+\delta_{y}\right)$ be two velocity vectors such that both $\mathbf{v}, \mathbf{v}_{\mathbf{p}} \in \mathcal{Z}_{1}$ or both $\mathbf{v}, \mathbf{v}_{\mathbf{p}} \in \mathcal{Z}_{2}$. Let $\mathbf{w}=(\dot{t}, \dot{z})$ and $\mathbf{w}_{\mathbf{p}}=\left(\dot{t}_{p}, \dot{z}_{p}\right)$ be the new computed velocity vectors starting from $\mathbf{v}$ and $\mathbf{v}_{\mathbf{p}}$, respectively. Then

i) $\left|\dot{t}_{p}-\dot{t}\right| \leq\left|\delta_{x}\right|+\left|\delta_{y}\right| \quad$ and $\quad\left|\dot{z}_{p}-\dot{z}\right| \leq\left|\delta_{x}\right|+\left|\delta_{y}\right|$;

ii) $\left|\left(k \dot{t}_{p}+\dot{z}_{p}\right)-(k \dot{t}+\dot{z})\right| \leq \frac{3}{2}\left|\delta_{x}\right|+\left|\delta_{y}\right|$ and

$$
\left|\left(k \dot{t}_{p}-\dot{z}_{p}\right)-(k \dot{t}-\dot{z})\right| \leq \frac{3}{2}\left|\delta_{x}\right|+\left|\delta_{y}\right| \text {. }
$$

Proof: If $\mathbf{v}, \mathbf{v}_{\mathbf{p}} \in \mathcal{Z}_{1}$ then, from (14b) we have

$\dot{t}_{p}-\dot{t}=\frac{\left(1-\varepsilon k^{2}\right)}{1+k^{2}} \delta_{x}+\frac{(1+\varepsilon) k}{1+k^{2}} \delta_{y} \quad$ and $\quad \dot{z}_{p}-\dot{z}=\frac{(1+\varepsilon) k}{1+k^{2}} \delta_{x}-\frac{\varepsilon-k^{2}}{1+k^{2}} \delta_{y}$. 
so that $i$ ) follows from $\varepsilon, k<1$. Moreover, we have that

$$
\begin{aligned}
\left(k \dot{t}_{p}+\dot{z}_{p}\right)-(k \dot{t}+\dot{z}) & =\frac{k\left(1-\varepsilon k^{2}\right)}{1+k^{2}} \delta_{x}+\frac{k^{2}(1+\varepsilon)}{1+k^{2}} \delta_{y}+\frac{k(1+\varepsilon)}{1+k^{2}} \delta_{x}-\frac{\varepsilon-k^{2}}{1+k^{2}} \delta_{y} \\
& =\frac{k\left(2-\varepsilon k^{2}+\varepsilon\right)}{1+k^{2}} \delta_{x}+\frac{\left(2 k^{2}+\varepsilon k^{2}-\varepsilon\right)}{1+k^{2}} \delta_{y}, \\
\left(k \dot{t}_{p}-\dot{z}_{p}\right)-(k \dot{t}-\dot{z}) & =\frac{k\left(1-\varepsilon k^{2}\right)}{1+k^{2}} \delta_{x}+\frac{k^{2}(1+\varepsilon)}{1+k^{2}} \delta_{y}-\frac{k(1+\varepsilon)}{1+k^{2}} \delta_{x}+\frac{\varepsilon-k^{2}}{1+k^{2}} \delta_{y} \\
& =-k \varepsilon \delta_{x}+\varepsilon \delta_{y} .
\end{aligned}
$$

Since $\varepsilon, k \leq 1$, we have

$$
\begin{aligned}
& 0 \leq \frac{k\left(2-\varepsilon k^{2}+\varepsilon\right)}{1+k^{2}}=\frac{k}{1+k^{2}}\left(2+\varepsilon\left(1-k^{2}\right)\right) \leq 3 \frac{k}{1+k^{2}} \leq \frac{3}{2} \quad \text { and } \\
& \left|\frac{\left(2 k^{2}+\varepsilon k^{2}-\varepsilon\right)}{1+k^{2}}\right|= \begin{cases}1 & \text { if } k=1, \\
\frac{2 k^{2}-\varepsilon\left(1-k^{2}\right)}{1+k^{2}} \leq \frac{2 k^{2}}{1+k^{2}} \leq 1 \quad \text { if } k \neq 1, \varepsilon \leq \frac{2 k^{2}}{1-k^{2}}, \\
\frac{\varepsilon\left(1-k^{2}\right)-2 k^{2}}{1+k^{2}} \leq \frac{\varepsilon\left(1-k^{2}\right)}{1+k^{2}} \leq 1 \quad \text { if } k \neq 1, \varepsilon>\frac{2 k^{2}}{1-k^{2}} .\end{cases}
\end{aligned}
$$

Then $i$ ) follows. Analogous computation holds when $\mathbf{v}$ and $\mathbf{v}_{\mathbf{p}}$ are in $\mathcal{Z}_{2}$.

Theorem 6.1 Starting from the same input, that is the same angle $\alpha$, the same parameter $\varepsilon$ and the same initial velocity vector $\left(\dot{x}_{0}, \dot{y}_{0}\right), T A$ and $N A$ either compute the same output in the same number of steps, or they compute slightly different outputs, even if one of the algorithms performs more steps.

Proof: First of all, we observe that, if NA stops because $\left\|\mathbf{v}_{f}\right\|_{2}<S_{v}$, then the disk is almost at rest and so, even if TA computes further iterations, its output is similar to the final velocity computed by NA. Let $\left(\dot{x}_{n}, \dot{y}_{n}\right)$ be the velocity vector, with corresponding $\dot{\xi}_{n}=k \dot{x}_{n}+\dot{y}_{n}$ and $\dot{\eta}_{n}=k \dot{x}_{n}-\dot{y}_{n}$, processed at the $n$-th step by both TA and NA. This is certainly verified at the first step, when $n=0$.

In the following cases both TA and NA have the same behavior, that is they compute the same velocity vector $\left(\dot{x}_{n+1}, \dot{y}_{n+1}\right)$.

- If $\dot{\xi}_{n} \leq 0$ and $\dot{\eta}_{n} \leq 0$ both algorithms stop. 
- If $\dot{\xi}_{n} \leq 0$ and $\dot{\eta}_{n} \geq S$, or $\dot{\xi}_{n} \geq S$ and $\dot{\eta}_{n} \leq 0$, or $\dot{\xi}_{n} \geq S$ and $\dot{\eta}_{n} \geq S$, both algorithms compute the same new velocity vector.

In the other cases, we show that the TA and NA have different behavior, but they compute similar outputs.

1. If $0<\dot{\xi}_{n}<S$ and $0<\dot{\eta}_{n}<S$, then NA stops and the disk can be considered almost at rest, since $k \gg S$ and

$$
\left\|\left(\dot{x}_{n}, \dot{y}_{n}\right)\right\|_{2}^{2}=\frac{1+k^{2}}{4 k^{2}}\left(\dot{\xi}_{n}^{2}+\dot{\eta}_{n}^{2}\right)+\frac{1-k^{2}}{2 k^{2}} \dot{\xi}_{n} \dot{\eta}_{n} \leq \frac{S^{2}}{k^{2}} .
$$

The TA consider $\left(\dot{x}_{n}, \dot{y}_{n}\right) \in \mathcal{Z}_{12}$ and computes a new iteration. Since the 2-norm of the velocity vector decreases at each step also the output of the TA corresponds to an almost at rest disk.

2. If $0<\dot{\xi}_{n}<S$ and $\dot{\eta}_{n} \leq 0$, then NA stops, while TA computes a new velocity vector $\left(\dot{x}_{n+1}, \dot{y}_{n+1}\right)$ using the relations in $\mathcal{Z}_{2}$. From Lemma 6.1. $\left(\dot{x}_{n+1}, \dot{y}_{n+1}\right)$ differs from $\left(\dot{x}_{n}, \dot{y}_{n}\right)$, component-wise, for less than $2 S$, since $0<\dot{\xi}_{n}<S$. Moreover, $\dot{\xi}_{n+1}=-\varepsilon \dot{\xi}_{n}$, that is $-S<\dot{\xi}_{n+1}<0$ and so, if $\dot{\eta}_{n+1}$ is negative, then TA stops and its output is similar to the one of NA. Otherwise, if $\dot{\eta}_{n+1}$ is positive, since $\dot{\eta}_{n} \leq 0$, we have

$$
0<\dot{\eta}_{n+1}=\dot{\eta}_{n}+(1+\varepsilon) \frac{1-k^{2}}{1+k^{2}} \dot{\xi}_{n}<(1+\varepsilon) \frac{1-k^{2}}{1+k^{2}} \dot{\xi}_{n}<2 S
$$

and so the disk is almost at rest, we conclude, as in item 1, that TA and NA produce similar outputs.

Analogously if $0<\dot{\eta}_{n}<S$ and $\dot{\xi}_{n} \leq 0$.

3. If $0<\dot{\xi}_{n} \leq S$ and $\dot{\eta}_{n}>S$, the NA and TA process the velocity vector $\left(\dot{x}_{n}, \dot{y}_{n}\right)$ in different ways. We have analogous behaviors if $\dot{\xi}_{n}>S$ and $0<\dot{\eta}_{n} \leq S$, changing the role of $\dot{\xi}_{n}$ and $\dot{\eta}_{n}$.

Let $0<\dot{\xi}_{n} \leq S$ and $\dot{\eta}_{n}>S$.

The NA computes $\left(\dot{x}_{n+1}^{(1)}, \dot{x}_{n+1}^{(1)}\right), \dot{\xi}_{n+1}^{(1)}=k \dot{x}_{n+1}^{(1)}+\dot{y}_{n+1}^{(1)}$ and $\dot{\eta}_{n+1}^{(1)}=$ $k \dot{x}_{n+1}^{(1)}-\dot{y}_{n+1}^{(1)}$, where

$$
\begin{aligned}
& \dot{x}_{n+1}^{(1)}=\frac{\dot{\xi}_{n+1}^{(1)}+\dot{\eta}_{n+1}^{(1)}}{2 k}=\frac{1}{2 k} \dot{\xi}_{n}+\frac{1-k^{2}-2 \varepsilon k^{2}}{2 k\left(1+k^{2}\right)} \dot{\eta}_{n} \\
& \dot{y}_{n+1}^{(1)}=\frac{\dot{\xi}_{n+1}^{(1)}-\dot{\eta}_{n+1}^{(1)}}{2}=\frac{1}{2} \dot{\xi}_{n}+\frac{1-k^{2}+2 \varepsilon}{2\left(1+k^{2}\right)} \dot{\eta}_{n} .
\end{aligned}
$$

Furthermore,

$\dot{\eta}_{n+1}^{(1)}=-\varepsilon \dot{\eta}_{n}<0 \quad$ and $\quad 0<\dot{\xi}_{n+1}^{(1)}=\dot{\xi}_{n}+\frac{(1+\varepsilon)\left(1-k^{2}\right)}{1+k^{2}} \dot{\eta}_{n}<S+2 \dot{\eta}_{n}$. 
The TA computes $\left(\dot{x}_{n+1}^{(12)}, \dot{x}_{n+1}^{(12)}\right), \dot{\xi}_{n+1}^{(12)}=k \dot{x}_{n+1}^{(12)}+\dot{y}_{n+1}^{(12)}$ and $\dot{\eta}_{n+1}^{(12)}=$ $k \dot{x}_{n+1}^{(12)}-\dot{y}_{n+1}^{(12)}$, where

$$
\begin{aligned}
& \dot{x}_{n+1}^{(12)}=\frac{\dot{\xi}_{n+1}^{(12)}+\dot{\eta}_{n+1}^{(12)}}{2 k}=\left(\dot{\xi}_{n}+\dot{\eta}_{n}\right) \frac{\left(1-k^{2}-2 \varepsilon k^{2}\right)\left(\dot{\xi}_{n}^{2}+\dot{\eta}_{n}^{2}\right)-2\left(1-k^{2}\right) \dot{\xi}_{n} \dot{\eta}_{n}}{8 k D} \\
& \dot{y}_{n+1}^{(12)}=\frac{\dot{\xi}_{n+1}^{(12)}-\dot{\eta}_{n+1}^{(12)}}{2}=\left(\dot{\eta}_{n}-\dot{\xi}_{n}\right) \frac{\left(1-k^{2}+2 \varepsilon\right)\left(\dot{\xi}_{n}^{2}+\dot{\eta}_{n}^{2}\right)+2\left(1-k^{2}\right) \dot{\xi}_{n} \dot{\eta}_{n}}{8 D}
\end{aligned}
$$

where $D=\frac{\left(\dot{\xi}_{n}^{2}+\dot{\eta}_{n}^{2}\right)\left(1+k^{2}\right)+2 \dot{\xi}_{n} \dot{\eta}_{n}\left(k^{2}-1\right)}{4}$. Furthermore,

$$
\dot{\eta}_{n+1}^{(12)}=\frac{-\varepsilon\left(\dot{\xi}_{n}^{2}+\dot{\eta}_{n}^{2}\right)\left(\dot{\eta}_{n}\left(1+k^{2}\right)-\dot{\xi}_{n}\left(1-k^{2}\right)\right)-\dot{\xi}_{n}\left(1-k^{2}\right)\left(\dot{\xi}_{n}^{2}+\dot{\eta}_{n}^{2}\right)}{4 D}
$$

and, since $\dot{\eta}_{n}>\dot{\xi}_{n}$, then also $\dot{\eta}_{n+1}^{(12)}<0$.

The linear velocities $\left(\dot{x}_{n+1}^{(1)}, \dot{y}_{n+1}^{(1)}\right)$ and $\left(\dot{x}_{n+1}^{(12)}, \dot{y}_{n+1}^{(12)}\right)$ are very similar. In fact, since $4 D=\left(\dot{\xi}_{n}^{2}+\dot{\eta}_{n}^{2}\right)\left(1+k^{2}\right)+2 \dot{\xi}_{n} \dot{\eta}_{n}\left(k^{2}-1\right) \approx \dot{\eta}_{n}^{2}\left(1+k^{2}\right)$,

$$
\begin{aligned}
\left|\dot{x}_{n+1}^{(1)}-\dot{x}_{n+1}^{(12)}\right| & =\frac{\dot{\xi}_{n} k(1+\varepsilon)\left(\dot{\eta}_{n}^{2}\left(3-k^{2}\right)+\dot{\xi}_{n}^{2}\left(1+k^{2}\right)\right)}{4 D\left(1+k^{2}\right)} \\
& \approx \frac{\dot{\xi}_{n} k(1+\varepsilon)\left(3-k^{2}\right)}{\left(1+k^{2}\right)^{2}}<2 \dot{\xi}_{n}<2 S \\
\left|\dot{y}_{n+1}^{(1)}-\dot{y}_{n+1}^{(12)}\right| & =\frac{\dot{\xi}_{n}(1+\varepsilon)\left|\dot{\eta}_{n}^{2}\left(3 k^{2}-1\right)+\dot{\xi}_{n}^{2}\left(1+k^{2}\right)\right|}{4 D\left(1+k^{2}\right)} \\
& \approx \frac{\dot{\xi}_{n}(1+\varepsilon)\left|3 k^{2}-1\right|}{\left(1+k^{2}\right)^{2}}<2 \dot{\xi}_{n}<2 S .
\end{aligned}
$$

It follows that

$$
\begin{aligned}
& \left|\dot{\xi}_{n+1}^{(1)}-\dot{\xi}_{n+1}^{(12)}\right|<|k|\left|\dot{x}_{n+1}^{(1)}-\dot{x}_{n+1}^{(12)}\right|+\left|\dot{y}_{n+1}^{(1)}-\dot{y}_{n+1}^{(12)}\right|<4 S \\
& \left|\dot{\eta}_{n+1}^{(1)}-\dot{\eta}_{n+1}^{(12)}\right|<|k|\left|\dot{x}_{n+1}^{(1)}-\dot{x}_{n+1}^{(12)}\right|+\left|\dot{y}_{n+1}^{(1)}-\dot{y}_{n+1}^{(12)}\right|<4 S .
\end{aligned}
$$

Summing up, we have

$$
\begin{gathered}
\dot{\xi}_{n+1}^{(1)}>0 \quad \text { and } \quad \dot{\eta}_{n+1}^{(1)}<0, \\
\dot{\xi}_{n+1}^{(1)}-4 S<\dot{\xi}_{n+1}^{(12)}<\dot{\xi}_{n+1}^{(1)}+4 S \quad \text { and } \quad \dot{\eta}_{n+1}^{(1)}-4 S<\dot{\eta}_{n+1}^{(12)}<0 .
\end{gathered}
$$


Since $\dot{\eta}_{n+1}^{(1)}, \dot{\eta}_{n+1}^{(12)}<0$, if one of TA and NA does not stop, then it computes a new velocity vector using the formulæ of $\mathcal{Z}_{2}$.

In general, there are the following cases.

a) Let $0<\dot{\xi}_{n+1}^{(1)} \leq S$ and $\dot{\xi}_{n+1}^{(12)} \leq 0$. NA and TA stop, since $\dot{\eta}_{n+1}^{(1)}, \dot{\eta}_{n+1}^{(12)}<$ 0, and they return the similar outputs $\left(\dot{x}_{n+1}^{(1)}, \dot{y}_{n+1}^{(1)}\right)$ and $\left(\dot{x}_{n+1}^{(12)}, \dot{y}_{n+1}^{(12)}\right)$.

b) Let $0<\dot{\xi}_{n+1}^{(1)} \leq S$ and $\dot{\xi}_{n+1}^{(12)}>0$. NA stops, since $\dot{\eta}_{n+1}^{(1)}<0$.

TA uses the formulæ in $\mathcal{Z}_{2}$, since $\dot{\eta}_{n+1}^{(12)}<0$. We have $0<\dot{\xi}_{n+1}^{(12)} \leq$ $\dot{\xi}_{n+1}^{(1)}+4 S<5 S$ and $\eta_{n+1}^{(12)}<0$, and so Lemma 6.1 implies that TA performs small changes to $\left(\dot{x}_{n+1}^{(12)}, \dot{y}_{n+1}^{(12)}\right)$.

Moreover, $\dot{\xi}_{n+2}=-\varepsilon \dot{\xi}_{n+1}^{(12)}$ and so $-5 S<\dot{\xi}_{n+2}<0$. If $\dot{\eta}_{n+2} \leq 0$, then TA stops with a similar output as NA. Otherwise, if $\dot{\eta}_{n+2}>$ 0 , then

$$
0<\dot{\eta}_{n+2}=\dot{\eta}_{n+1}^{(12)}+(1+\varepsilon) \frac{1-k^{2}}{1+k^{2}} \dot{\xi}_{n+1}^{(12)}
$$

and so, since $\dot{\eta}_{n+1}^{(12)}<0$,

$$
\left.0<-\dot{\eta}_{n+1}^{(12)}<(1+\varepsilon) \frac{1-k^{2}}{1+k^{2}} \dot{\xi}_{n+1}^{(12}\right)<10 S .
$$

In this case, since $\dot{\xi}_{n+1}^{(12)}$ and $\dot{\eta}_{n+1}^{(12)}$ are very small, the disk, at the $(n+1)$-th step, is almost at rest and the output of TA and NA is very similar, independently of the number of steps performed by TA, after NA has stopped.

c) Let $\xi_{n+1}^{(1)}>S$ and $\dot{\xi}_{n+1}^{(12)} \leq 0$. TA stops, since $\eta_{n+1}^{(12)}<0$. NA uses the formulæ in $\mathcal{Z}_{2}$. Since $0>\xi_{n+1}^{(12)}>\xi_{n+1}^{(1)}-4 S$, then $S<$ $\xi_{n+1}^{(1)}<4 S$ and, from Lemma 6.1. NA performs small changes to $\left(\dot{x}_{n+1}^{(1)}, \dot{y}_{n+1}^{(1)}\right)$.

Since $\dot{\xi}_{n+2}=-\varepsilon \dot{\xi}_{n+1}^{(1)}$, we have $-4 S<\dot{\xi}_{n+2}<0$ and, if $\dot{\eta}_{n+2} \leq 0$, then NA stops and it returns a final velocity similar to the output of TA. Otherwise, if $\dot{\eta}_{n+2}>0$, since $\dot{\eta}_{n+1}^{(1)}<0$, we have that

$$
0<-\dot{\eta}_{n+1}^{(1)}<(1+\varepsilon) \frac{1-k^{2}}{1+k^{2}} \dot{\xi}_{n+1}^{(1)}<8 S .
$$

In this case, since $\dot{\xi}_{n+1}^{(11)}$ and $\dot{\eta}_{n+1}^{(12)}$ are very small, the disk, at the $(n+1)$-th step, is almost at rest and the output of TA and NA is very similar, independently of the number of steps performed by NA, after TA has stopped. 
d) Let $\dot{\xi}_{n+1}^{(1)}>S$ and $\dot{\xi}_{n+1}^{(12)}>0$. Both TA and NA compute, using the same formulæ in $\mathcal{Z}_{2}$, a new iteration starting from two similar velocities. Lemma 6.2 implies that the new computed velocities slightly differ from each other. Moreover, the new values of $\dot{\xi}$, equal to $-\varepsilon \dot{\xi}_{n+1}^{(1)}$ and $-\varepsilon \dot{\xi}_{n+1}^{(12)}$ respectively, are negative, so that we can repeat an analysis of the behavior of TA and NA analogous to the one presented in items a $-\mathrm{d}$, changing the role of $\dot{\xi}$ and $\dot{\eta}$.

Table 1 illustrates the possible different cases after the $n$-th step.

\begin{tabular}{|c|c|c|c|}
\cline { 2 - 4 } \multicolumn{1}{c|}{} & $\dot{\xi}_{n+1} \leq 0$ & $0<\dot{\xi}_{n+1}<S$ & $\dot{\xi}_{n+1} \geq S$ \\
\hline$\dot{\eta}_{n+1} \leq 0$ & TA and NA & TA small changes & TA and NA \\
& stop & NA stops & in $\mathcal{Z}_{2}$ \\
\hline $0<\dot{\eta}_{n+1}<S$ & TA small changes & TA disk almost at rest & TA in $\mathcal{Z}_{12}$ \\
& NA stops & NA stops & NA in $\mathcal{Z}_{2}$ \\
\hline$\dot{\eta}_{n+1} \geq S$ & TA and NA & TA in $\mathcal{Z}_{12}$ & TA and NA \\
& in $\mathcal{Z}_{1}$ & NA in $\mathcal{Z}_{1}$ & in $\mathcal{Z}_{12}$ \\
\hline
\end{tabular}

Table 1: Behavior of TA and NA, starting from the same $\left(\dot{\xi}_{n}, \dot{\eta}_{n}\right)$.

In conclusion, the following cases happen:

1. both algorithms have the same behavior (same iterations and same steps number);

2. NA stops and TA does not stop and it makes small changes computing the new velocities or vice versa;

3. NA stops and TA does not stop, but the disk is almost at rest;

4. both algorithms computes velocities whose difference is very small.

\subsection{Numerical examples}

We consider several examples, obtained by varying the coefficient $\varepsilon$ and the angle $\alpha$. Furthermore, for each pair $(\varepsilon, \alpha)$, we consider several initial linear velocity $\mathbf{v}_{0}=\left(\dot{x}_{0}, \dot{y}_{0}\right)$, with $\left\|\mathbf{v}_{0}\right\|_{2}=1$. The following Table 2 shows some possible values of $\varepsilon$ and $\alpha$ and, denoting by $k=\tan \alpha$, some initial velocities $\widehat{\mathbf{v}}_{0}$ such that $\mathbf{v}_{0}=\frac{\widehat{\mathbf{v}}_{0}}{\left\|\widehat{\mathbf{v}}_{0}\right\|_{2}}$. 


\begin{tabular}{|c||c|c|c|c|c|c|c|}
\hline \multicolumn{7}{|c|}{ Values of $\varepsilon$} \\
\hline$\#$ & 1 & 2 & 3 & 4 & 5 & 6 & 7 \\
\hline$\varepsilon$ & 1 & 0.95 & 0.75 & 0.5 & 0.25 & 0.05 & 0 \\
\hline \hline \multicolumn{8}{|c|}{ Values of $\alpha$} \\
\hline$\#$ & 1 & 2 & 3 & 4 & 5 & 6 & 7 \\
\hline$\alpha$ & $\pi / 4$ & $\pi / 6$ & $\pi / 8$ & $\pi / 12$ & $\pi / 16$ & $\pi / 32$ & $\pi / 64$ \\
\hline \hline \multicolumn{8}{|c|}{ Values of $\widehat{\mathbf{v}}_{0}$} \\
\hline$\#$ & 1 & 2 & 3 & 4 & 5 & 6 & 7 \\
\hline$\widehat{\mathbf{v}}_{0}$ & $(1,0)$ & $(1, k / 3)$ & $(1,2 k / 3)$ & $(1, k)$ & $(1,1 / k)$ & $(0,1)$ & $(-1, k)$ \\
\hline
\end{tabular}

Table 2: Coefficients, angles, initial velocity

The following tables collect the results of NA corresponding to pairs $(\varepsilon, \alpha)$. The entries of each line of a table show, respectively, the number of the example, the initial velocity vector $\mathbf{v}_{0}$, the final velocity vector $\mathbf{v}_{f}$ and its norm, the case to which $\mathbf{v}_{0}$ belongs, the number $N$ of steps to obtain $\mathbf{v}_{f}$ and if the algorithm stops because $\mathbf{v}_{f} \in \mathcal{Z}_{0}$ or because $\left\|\mathbf{v}_{f}\right\|_{2}<S_{v}$.

The results are obtained processing the previous data by the NA, implemented in MatLab, using $S=2 \cdot 2^{-52}=4.44 \cdot 10^{-16}$, which corresponds to twice the machine precision, $S_{v}=10^{-12}$, and the maximum number of step $N_{\max }=10^{4}$. Note that a similar threshold $S_{v}$ ensure that, with an input velocity of 1 kilometer/second, the rest condition is fixed for an output velocity of less than 1 nanometer/second.

Later on we denote with $i . j . k$ the example where $\varepsilon$ assumes the $i$-th value, $\alpha$ assumes the $j$-th value and $v_{0}$ the $k$-th value of the Table 2 , e.g. the case 3.2 is obtained using $\varepsilon=0.75$ and $\alpha=\pi / 6$ and the case 6.4 .2 is obtained using $\varepsilon=0.05, \alpha=\pi / 12$ and $\widehat{\mathbf{v}}_{0}=(1, k / 3)$.

The cases **.1 and *.*.7 are test situations: in fact all the cases *.*.1 are such that $\mathbf{v}_{0}$ has the direction of the angle bisector and the behavior of the algorithm is known from theoretical results; all the cases **.7 are such that $\mathbf{v}_{0} \in \mathcal{Z}_{0}$ and then $\mathbf{v}_{0}$ is immediately the output velocity. Moreover, the cases *.*.2 and *.*.3 are the only ones with multiple impact. In the case *.*.4 the disk moves along in contact with one wall and impacts with the other. In the case **.5 the initial velocity is orthogonal to one wall. In the case **.6 the initial velocity is orthogonal to the bisector of the angle.

The cases $* .1$. $^{*}$ too are test situations, since when $\alpha=\pi / 4$ or, that is the same, $k=1$, once again the behavior of the algorithm is known from theoretical results.

The case $7 .^{*} .^{*}$, such that $\varepsilon=0$, does not imply that the disk stops after the first impact, since only that the orthogonal component of the velocity with respect to the impacted wall is annihilated.

Remark 10. The case 3.7.*, where $\alpha=\pi / 64$, is the first case for which, in 
perfect agreement with the theoretical considerations about the algorithm, the algorithm stops because of the presence of the threshold $S_{v}$ on the norm of $\mathbf{v}_{n}$. Moreover, the case $3 .^{*}{ }^{*}$, where $\varepsilon=0.75$, is the first where it becomes more evident that, once again in perfect agreement with the theoretical considerations about the algorithm, the number of steps of the algorithm increases when the angle $\alpha$ between the walls becomes smaller. Both this events are more highlighted by the subsequent examples.

Ideal case: $\varepsilon=1$.

\begin{tabular}{|c||c||c||c||c||c||c|}
\hline \multicolumn{7}{|c|}{$\alpha=\pi / 4=0.78540 \quad \Rightarrow \quad \dot{\mathcal{Z}}^{\prime}$} \\
\hline$\#$ & $\left(\dot{x}_{0}, \dot{y}_{0}\right)$ & $\left(\mathbf{v}_{f}, \dot{y}_{f}\right)$ & $N$ & Stop \\
\hline 1.1 .1 & $(1.000,0.000)$ & $(-1.00 \mathrm{e}+00,-0.00 \mathrm{e}+00)$ & 1 & $\mathcal{Z}_{12}$ & 1 & $\mathcal{Z}_{0}$ \\
1.1 .2 & $(0.949,0.316)$ & $(-9.49 \mathrm{e}-01,-3.16 \mathrm{e}-01)$ & 1 & $\mathcal{Z}_{12}$ & 1 & $\mathcal{Z}_{0}$ \\
1.1 .3 & $(0.832,0.555)$ & $(-8.32 \mathrm{e}-01,-5.55 \mathrm{e}-01)$ & 1 & $\mathcal{Z}_{12}$ & 1 & $\mathcal{Z}_{0}$ \\
1.1 .4 & $(0.707,0.707)$ & $(-7.07 \mathrm{e}-01,-7.07 \mathrm{e}-01)$ & 1 & $\mathcal{Z}_{2}$ & 1 & $\mathcal{Z}_{0}$ \\
1.1 .5 & $(0.707,0.707)$ & $(-7.07 \mathrm{e}-01,-7.07 \mathrm{e}-01)$ & 1 & $\mathcal{Z}_{2}$ & 1 & $\mathcal{Z}_{0}$ \\
1.1 .6 & $(0.000,1.000)$ & $(-1.00 \mathrm{e}+00,-1.11 \mathrm{e}-16)$ & 1 & $\mathcal{Z}_{2}$ & 1 & $\mathcal{Z}_{0}$ \\
1.1 .7 & $(-0.707,0.707)$ & $(-7.07 \mathrm{e}-01,7.07 \mathrm{e}-01)$ & 1 & $\mathcal{Z}_{0}$ & 0 & $\mathcal{Z}_{0}$ \\
\hline
\end{tabular}

\begin{tabular}{|c||c||c||c||c||c||c|}
\hline \multicolumn{7}{|c|}{$\alpha=\pi / 6=0.52360 \quad \Rightarrow \quad k=0.57735$} \\
\hline$\#$ & $\left(\dot{x}_{0}, \dot{y}_{0}\right)$ & $\left(\dot{x}_{f}, \dot{y}_{f}\right)$ & $\left\|\mathbf{v}_{f}\right\|$ & $\mathcal{Z}\left(\mathbf{v}_{0}\right)$ & $N$ & Stop \\
\hline 1.2 .1 & $(1.000,0.000)$ & $(-1.00 \mathrm{e}+00,-0.00 \mathrm{e}+00)$ & 1 & $\mathcal{Z}_{12}$ & 1 & $\mathcal{Z}_{0}$ \\
1.2 .2 & $(0.982,0.189)$ & $(-9.82 \mathrm{e}-01,-1.89 \mathrm{e}-01)$ & 1 & $\mathcal{Z}_{12}$ & 2 & $\mathcal{Z}_{0}$ \\
1.2 .3 & $(0.933,0.359)$ & $(-9.55 \mathrm{e}-01,2.95 \mathrm{e}-01)$ & 1 & $\mathcal{Z}_{12}$ & 2 & $\mathcal{Z}_{0}$ \\
1.2 .4 & $(0.866,0.500)$ & $(-8.66 \mathrm{e}-01,5.00 \mathrm{e}-01)$ & 1 & $\mathcal{Z}_{2}$ & 2 & $\mathcal{Z}_{0}$ \\
1.2 .5 & $(0.500,0.866)$ & $(-1.00 \mathrm{e}+00,8.33 \mathrm{e}-17)$ & 1 & $\mathcal{Z}_{2}$ & 2 & $\mathcal{Z}_{0}$ \\
1.2 .6 & $(0.000,1.000)$ & $(-8.66 \mathrm{e}-01,-5.00 \mathrm{e}-01)$ & 1 & $\mathcal{Z}_{2}$ & 1 & $\mathcal{Z}_{0}$ \\
1.2 .7 & $(-0.866,0.500)$ & $(-8.66 \mathrm{e}-01,5.00 \mathrm{e}-01)$ & 1 & $\mathcal{Z}_{0}$ & 0 & $\mathcal{Z}_{0}$ \\
\hline
\end{tabular}

\begin{tabular}{|c||c||c||c||c||c||c|}
\hline \multicolumn{7}{|c|}{$\alpha=\pi / 8=0.39270 \quad \Rightarrow \quad k=0.41421$} \\
\hline$\#$ & $\left(\dot{x}_{0}, \dot{y}_{0}\right)$ & $\left(\dot{x}_{f}, \dot{y}_{f}\right)$ & $\left\|\mathbf{v}_{f}\right\|$ & $\mathcal{Z}\left(\mathbf{v}_{0}\right)$ & $N$ & Stop \\
\hline 1.3 .1 & $(1.000,0.000)$ & $(-1.00 \mathrm{e}+00,-0.00 \mathrm{e}+00)$ & 1 & $\mathcal{Z}_{12}$ & 1 & $\mathcal{Z}_{0}$ \\
1.3 .2 & $(0.991,0.137)$ & $(-9.38 \mathrm{e}-01,3.45 \mathrm{e}-01)$ & 1 & $\mathcal{Z}_{12}$ & 3 & $\mathcal{Z}_{0}$ \\
1.3 .3 & $(0.964,0.266)$ & $(-9.82 \mathrm{e}-01,-1.88 \mathrm{e}-01)$ & 1 & $\mathcal{Z}_{12}$ & 3 & $\mathcal{Z}_{0}$ \\
1.3 .4 & $(0.924,0.383)$ & $(-9.24 \mathrm{e}-01,-3.83 \mathrm{e}-01)$ & 1 & $\mathcal{Z}_{2}$ & 3 & $\mathcal{Z}_{0}$ \\
1.3 .5 & $(0.383,0.924)$ & $(-9.24 \mathrm{e}-01,3.83 \mathrm{e}-01)$ & 1 & $\mathcal{Z}_{2}$ & 2 & $\mathcal{Z}_{0}$ \\
1.3 .6 & $(0.000,1.000)$ & $(-1.00 \mathrm{e}+00,0.00 \mathrm{e}+00)$ & 1 & $\mathcal{Z}_{2}$ & 2 & $\mathcal{Z}_{0}$ \\
1.3 .7 & $(-0.924,0.383)$ & $(-9.24 \mathrm{e}-01,3.83 \mathrm{e}-01)$ & 1 & $\mathcal{Z}_{0}$ & 0 & $\mathcal{Z}_{0}$ \\
\hline
\end{tabular}




\begin{tabular}{|c|c|c|c|c|c|c|}
\hline \multicolumn{7}{|c|}{$\alpha=\pi / 12=0.26180$} \\
\hline \# & $\left(\dot{x}_{0}, \dot{y}_{0}\right)$ & $\left(\dot{x}_{f}, \dot{y}_{f}\right)$ & $\mid \mathbf{v}_{f} \|$ & $\mathcal{Z}\left(\mathbf{v}_{0}\right)$ & $N$ & Stop \\
\hline 1.4 .1 & $(1.000,0.000)$ & $(-1.00 \mathrm{e}+00,-0.00 \mathrm{e}+00)$ & 1 & $\mathcal{Z}_{12}$ & 1 & $\mathcal{Z}_{0}$ \\
\hline 1.4 .2 & $(0.996,0.089)$ & $(-9.92 \mathrm{e}-01,1.27 \mathrm{e}-01)$ & 1 & $\mathcal{Z}_{12}$ & 4 & $\mathcal{Z}_{0}$ \\
\hline 1.4 .3 & $(0.984,0.176)$ & $(-9.94 \mathrm{e}-01,-1.06 \mathrm{e}-01)$ & 1 & $\mathcal{Z}_{12}$ & 5 & $\mathcal{Z}_{0}$ \\
\hline 1.4 .4 & $(0.966,0.259)$ & $(-9.66 \mathrm{e}-01,-2.59 \mathrm{e}-01)$ & 1 & $\mathcal{Z}_{2}$ & 5 & $\mathcal{Z}_{0}$ \\
\hline 1.4 .5 & $(0.259,0.966)$ & $(-9.66 \mathrm{e}-01,-2.59 \mathrm{e}-01)$ & 1 & $\mathcal{Z}_{2}$ & 3 & $\mathcal{Z}_{0}$ \\
\hline 1.4.6 & $(0.000,1.000)$ & $(-1.00 \mathrm{e}+00,-1.04 \mathrm{e}-16)$ & 1 & $\mathcal{Z}_{2}$ & 3 & $\mathcal{Z}_{0}$ \\
\hline 1.4.7 & $(-0.966,0.259)$ & $(-9.66 \mathrm{e}-01,2.59 \mathrm{e}-01)$ & 1 & $\mathcal{Z}_{0}$ & 0 & $\mathcal{Z}_{0}$ \\
\hline
\end{tabular}

\begin{tabular}{|c|c|c|c|c|c|c|}
\hline \multicolumn{7}{|c|}{$\alpha=\pi / 16=0.19635$} \\
\hline \# & $\left(\dot{x}_{0}, \dot{y}_{0}\right)$ & $\left(\dot{x}_{f}, \dot{y}_{f}\right)$ & $\mid \mathbf{v}_{f} \|$ & $\mathcal{Z}\left(\mathbf{v}_{0}\right)$ & $N$ & Stop \\
\hline 1.5 .1 & $(1.000,0.000)$ & $(-1.00 \mathrm{e}+00,-0.00 \mathrm{e}+00$ & 1 & $\mathcal{Z}_{12}$ & 1 & $\mathcal{Z}_{0}$ \\
\hline 1.5 .2 & $(0.998,0.066)$ & $(-9.99 \mathrm{e}-01,3.58 \mathrm{e}-02)$ & 1 & $\mathcal{Z}_{12}$ & 6 & $\mathcal{Z}_{0}$ \\
\hline 1.5 .3 & $(0.991,0.131)$ & $(-9.97 \mathrm{e}-01,-7.36 \mathrm{e}-02)$ & 1 & $\mathcal{Z}_{12}$ & 7 & $\mathcal{Z}_{0}$ \\
\hline 1.5 .4 & $(0.981,0.195)$ & $(-9.81 \mathrm{e}-01,-1.95 \mathrm{e}-01)$ & 1 & $\mathcal{Z}_{2}$ & 7 & $\mathcal{Z}_{0}$ \\
\hline 1.5.5 & $(0.195,0.981)$ & $(-9.81 \mathrm{e}-01,1.95 \mathrm{e}-01)$ & 1 & $\mathcal{Z}_{2}$ & 4 & $\mathcal{Z}_{0}$ \\
\hline 1.5 .6 & $(0.000,1.000)$ & $(-1.00 \mathrm{e}+00,0.00 \mathrm{e}+00$ & 1 & $\mathcal{Z}_{2}$ & 4 & $\mathcal{Z}_{0}$ \\
\hline 1.5 .7 & $(-0.981,0.195)$ & $(-9.81 \mathrm{e}-01,1.95 \mathrm{e}-01)$ & 1 & $\mathcal{Z}_{0}$ & 0 & $\mathcal{Z}_{0}$ \\
\hline
\end{tabular}

\begin{tabular}{|c||c||c||c||c||c||c|}
\hline \multicolumn{7}{|c|}{$\alpha=\pi / 32=0.09817 \quad \Rightarrow \quad k=0.09849$} \\
\hline$\#$ & $\left(\dot{x}_{0}, \dot{y}_{0}\right)$ & $\left(\dot{x}_{f}, \dot{y}_{f}\right)$ & $\left\|\mathbf{v}_{f}\right\|$ & $\mathcal{Z}\left(\mathbf{v}_{0}\right)$ & $N$ & Stop \\
\hline 1.6 .1 & $(1.000,0.000)$ & $(-1.00 \mathrm{e}+00,-0.00 \mathrm{e}+00)$ & 1 & $\mathcal{Z}_{12}$ & 1 & $\mathcal{Z}_{0}$ \\
1.6 .2 & $(0.999,0.033)$ & $(-1.00 \mathrm{e}+00,-1.84 \mathrm{e}-02)$ & 1 & $\mathcal{Z}_{12}$ & 14 & $\mathcal{Z}_{0}$ \\
1.6 .3 & $(0.998,0.066)$ & $(-9.99 \mathrm{e}-01,-3.38 \mathrm{e}-02)$ & 1 & $\mathcal{Z}_{12}$ & 15 & $\mathcal{Z}_{0}$ \\
1.6 .4 & $(0.995,0.098)$ & $(-9.95 \mathrm{e}-01,-9.80 \mathrm{e}-02)$ & 1 & $\mathcal{Z}_{2}$ & 15 & $\mathcal{Z}_{0}$ \\
1.6 .5 & $(0.098,0.995)$ & $(-9.95 \mathrm{e}-01,9.80 \mathrm{e}-02)$ & 1 & $\mathcal{Z}_{2}$ & 8 & $\mathcal{Z}_{0}$ \\
1.6 .6 & $(0.000,1.000)$ & $(-1.00 \mathrm{e}+00,8.25 \mathrm{e}-17)$ & 1 & $\mathcal{Z}_{2}$ & 8 & $\mathcal{Z}_{0}$ \\
1.6 .7 & $(-0.995,0.098)$ & $(-9.95 \mathrm{e}-01,9.80 \mathrm{e}-02)$ & 1 & $\mathcal{Z}_{0}$ & 0 & $\mathcal{Z}_{0}$ \\
\hline
\end{tabular}

\begin{tabular}{|c||c||c||c||c||c||c|}
\hline \multicolumn{7}{|c|}{$\alpha=\pi / 64=0.04909 \quad \Rightarrow \quad{ }^{2}$} \\
\hline$\#$ & $\left(\dot{x}_{0}, \dot{y}_{0}\right)$ & $\left(\dot{x}_{f}, \dot{y}_{f}\right)$ & $\left\|\mathbf{v}_{f}\right\|$ & $\mathcal{Z}\left(\mathbf{v}_{0}\right)$ & $N$ & Stop \\
\hline 1.7 .1 & $(1.000,0.000)$ & $(-1.00 \mathrm{e}+00,-0.00 \mathrm{e}+00)$ & 1 & $\mathcal{Z}_{12}$ & 1 & $\mathcal{Z}_{0}$ \\
1.7 .2 & $(1.000,0.016)$ & $(-1.00 \mathrm{e}+00,-1.45 \mathrm{e}-02)$ & 1 & $\mathcal{Z}_{12}$ & 30 & $\mathcal{Z}_{0}$ \\
1.7 .3 & $(0.999,0.033)$ & $(-1.00 \mathrm{e}+00,-1.65 \mathrm{e}-02)$ & 1 & $\mathcal{Z}_{12}$ & 31 & $\mathcal{Z}_{0}$ \\
1.7 .4 & $(0.999,0.049)$ & $(-9.99 \mathrm{e}-01,-4.91 \mathrm{e}-02)$ & 1 & $\mathcal{Z}_{2}$ & 31 & $\mathcal{Z}_{0}$ \\
1.7 .5 & $(0.049,0.999)$ & $(-9.99 \mathrm{e}-01,4.91 \mathrm{e}-02)$ & 1 & $\mathcal{Z}_{2}$ & 16 & $\mathcal{Z}_{0}$ \\
1.7 .6 & $(0.000,1.000)$ & $(-1.00 \mathrm{e}+00,4.15 \mathrm{e}-17)$ & 1 & $\mathcal{Z}_{2}$ & 16 & $\mathcal{Z}_{0}$ \\
1.7 .7 & $(-0.999,0.049)$ & $(-9.99 \mathrm{e}-01,4.91 \mathrm{e}-02)$ & 1 & $\mathcal{Z}_{0}$ & 0 & $\mathcal{Z}_{0}$ \\
\hline
\end{tabular}


Non ideal case: $\varepsilon=0.95$.

\begin{tabular}{|c|c|c|c|c|c|c|}
\hline \multicolumn{7}{|c|}{$\alpha=\pi / 4=0.78540$} \\
\hline \# & $\left(\dot{x}_{0}, \dot{y}_{0}\right)$ & $\left(\dot{x}_{f}, \dot{y}_{f}\right)$ & $\left\|\mathbf{v}_{f}\right\|$ & $\mathcal{Z}\left(\mathbf{v}_{0}\right)$ & $N$ & Stop \\
\hline 2.1 .1 & $(1.000,0.000)$ & $(-9.50 \mathrm{e}-01,-0.00 \mathrm{e}+00)$ & $9.50 \mathrm{e}-01$ & $\mathcal{Z}_{12}$ & 1 & $\mathcal{Z}_{0}$ \\
\hline 2.1 .2 & $(0.949,0.316)$ & $(-9.01 \mathrm{e}-01,-3.00 \mathrm{e}-01)$ & $9.50 \mathrm{e}-01$ & $\mathcal{Z}_{12}$ & 1 & $\mathcal{Z}_{0}$ \\
\hline 2.1 .3 & $(0.832,0.555)$ & $(-7.90 \mathrm{e}-01,-5.27 \mathrm{e}-01)$ & $9.50 \mathrm{e}-01$ & $\mathcal{Z}_{12}$ & 1 & $\mathcal{Z}_{0}$ \\
\hline 2.1 .4 & $(0.707,0.707)$ & $(-6.72 \mathrm{e}-01,-6.72 \mathrm{e}-01)$ & $9.50 \mathrm{e}-01$ & $\mathcal{Z}_{2}$ & 1 & $\mathcal{Z}_{0}$ \\
\hline 2.1 .5 & $(0.707,0.707)$ & $(-6.72 \mathrm{e}-01,-6.72 \mathrm{e}-01)$ & $9.50 \mathrm{e}-01$ & $\mathcal{Z}_{2}$ & 1 & $\mathcal{Z}_{0}$ \\
\hline 2.1 .6 & $(0.000,1.000)$ & $(-9.75 \mathrm{e}-01,2.50 \mathrm{e}-02)$ & $9.75 \mathrm{e}-01$ & $\mathcal{Z}_{2}$ & 1 & $\mathcal{Z}_{0}$ \\
\hline 2.1 .7 & $(-0.707,0.707)$ & $(-7.07 \mathrm{e}-01,7.07 \mathrm{e}-01)$ & $1.00 \mathrm{e}+00$ & $\mathcal{Z}_{0}$ & 0 & $\mathcal{Z}_{0}$ \\
\hline
\end{tabular}

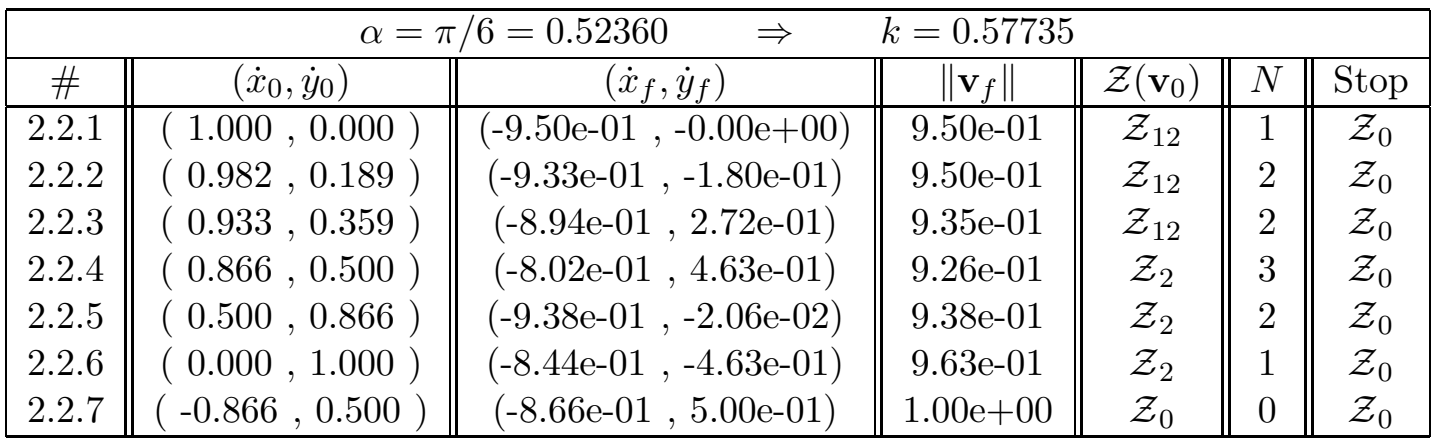

\begin{tabular}{|c||c||c||c||c||c||c|}
\hline \multicolumn{7}{|c|}{$\alpha=\pi / 8=0.39270 \quad \Rightarrow \quad k=0.41421$} \\
\hline$\#$ & $\left(\dot{x}_{0}, \dot{y}_{0}\right)$ & $\left(\dot{x}_{f}, \dot{y}_{f}\right)$ & $\left\|\mathbf{v}_{f}\right\|$ & $\mathcal{Z}\left(\mathbf{v}_{0}\right)$ & $N$ & Stop \\
\hline 2.3 .1 & $(1.000,0.000)$ & $(-9.50 \mathrm{e}-01,-0.00 \mathrm{e}+00)$ & $9.50 \mathrm{e}-01$ & $\mathcal{Z}_{12}$ & 1 & $\mathcal{Z}_{0}$ \\
2.3 .2 & $(0.991,0.137)$ & $(-8.77 \mathrm{e}-01,3.28 \mathrm{e}-01)$ & $9.37 \mathrm{e}-01$ & $\mathcal{Z}_{12}$ & 3 & $\mathcal{Z}_{0}$ \\
2.3 .3 & $(0.964,0.266)$ & $(-8.97 \mathrm{e}-01,-1.65 \mathrm{e}-01)$ & $9.12 \mathrm{e}-01$ & $\mathcal{Z}_{12}$ & 3 & $\mathcal{Z}_{0}$ \\
2.3 .4 & $(0.924,0.383)$ & $(-8.34 \mathrm{e}-01,-3.44 \mathrm{e}-01)$ & $9.02 \mathrm{e}-01$ & $\mathcal{Z}_{2}$ & 4 & $\mathcal{Z}_{0}$ \\
2.3 .5 & $(0.383,0.924)$ & $(-8.65 \mathrm{e}-01,3.33 \mathrm{e}-01)$ & $9.27 \mathrm{e}-01$ & $\mathcal{Z}_{2}$ & 2 & $\mathcal{Z}_{0}$ \\
2.3 .6 & $(0.000,1.000)$ & $(-9.51 \mathrm{e}-01,-3.38 \mathrm{e}-02)$ & $9.51 \mathrm{e}-01$ & $\mathcal{Z}_{2}$ & 2 & $\mathcal{Z}_{0}$ \\
2.3 .7 & $(-0.924,0.383)$ & $(-9.24 \mathrm{e}-01,3.83 \mathrm{e}-01)$ & $1.00 \mathrm{e}+00$ & $\mathcal{Z}_{0}$ & 0 & $\mathcal{Z}_{0}$ \\
\hline
\end{tabular}




\begin{tabular}{|c||c||c||c||c||c||c|}
\hline \multicolumn{7}{|c|}{$\alpha=\pi / 12=0.26180 \quad \Rightarrow \quad k=0.26795$} \\
\hline$\#$ & $\left(\dot{x}_{0}, \dot{y}_{0}\right)$ & $\left(\dot{x}_{f}, \dot{y}_{f}\right)$ & $\left\|\mathbf{v}_{f}\right\|$ & $\mathcal{Z}\left(\mathbf{v}_{0}\right)$ & $N$ & Stop \\
\hline 2.4 .1 & $(1.000,0.000)$ & $(-9.50 \mathrm{e}-01,-0.00 \mathrm{e}+00)$ & $9.50 \mathrm{e}-01$ & $\mathcal{Z}_{12}$ & 1 & $\mathcal{Z}_{0}$ \\
2.4 .2 & $(0.996,0.089)$ & $(-8.87 \mathrm{e}-01,9.37 \mathrm{e}-02)$ & $8.92 \mathrm{e}-01$ & $\mathcal{Z}_{12}$ & 4 & $\mathcal{Z}_{0}$ \\
2.4 .3 & $(0.984,0.176)$ & $(-8.59 \mathrm{e}-01,-8.55 \mathrm{e}-02)$ & $8.63 \mathrm{e}-01$ & $\mathcal{Z}_{12}$ & 5 & $\mathcal{Z}_{0}$ \\
2.4 .4 & $(0.966,0.259)$ & $(-8.29 \mathrm{e}-01,-2.19 \mathrm{e}-01)$ & $8.57 \mathrm{e}-01$ & $\mathcal{Z}_{2}$ & 6 & $\mathcal{Z}_{0}$ \\
2.4 .5 & $(0.259,0.966)$ & $(-8.83 \mathrm{e}-01,-1.96 \mathrm{e}-01)$ & $9.04 \mathrm{e}-01$ & $\mathcal{Z}_{2}$ & 3 & $\mathcal{Z}_{0}$ \\
2.4 .6 & $(0.000,1.000)$ & $(-9.27 \mathrm{e}-01,4.60 \mathrm{e}-02)$ & $9.28 \mathrm{e}-01$ & $\mathcal{Z}_{2}$ & 3 & $\mathcal{Z}_{0}$ \\
2.4 .7 & $(-0.966,0.259)$ & $(-9.66 \mathrm{e}-01,2.59 \mathrm{e}-01)$ & $1.00 \mathrm{e}+00$ & $\mathcal{Z}_{0}$ & 0 & $\mathcal{Z}_{0}$ \\
\hline
\end{tabular}

\begin{tabular}{|c|c|c|c|c|c|c|}
\hline \multicolumn{3}{|c|}{$\alpha=\pi / 16=0.19635$} & \multicolumn{4}{|c|}{$k=0.19891$} \\
\hline \# & $\left(\dot{x}_{0}, \dot{y}_{0}\right)$ & $\left(\dot{x}_{f}, \dot{y}_{f}\right)$ & $\left\|\mathbf{v}_{f}\right\|$ & $\mathcal{Z}\left(\mathbf{v}_{0}\right)$ & $N$ & Stop \\
\hline 2.5 .1 & $(1.000,0.000)$ & $(-9.50 \mathrm{e}-01,-0.00 \mathrm{e}+00)$ & $9.50 \mathrm{e}-01$ & $\mathcal{Z}_{12}$ & 1 & $\mathcal{Z}_{0}$ \\
\hline 2.5 .2 & $(0.998,0.066)$ & $(-8.42 \mathrm{e}-01,7.41 \mathrm{e}-03)$ & $8.42 \mathrm{e}-01$ & $\mathcal{Z}_{12}$ & 6 & $\mathcal{Z}_{0}$ \\
\hline 2.5 .3 & $(0.991,0.131)$ & $(-8.16 \mathrm{e}-01,-5.77 \mathrm{e}-02)$ & $8.18 \mathrm{e}-01$ & $\mathcal{Z}_{12}$ & 7 & $\mathcal{Z}_{0}$ \\
\hline 2.5 .4 & $(0.981,0.195)$ & $(-8.00 \mathrm{e}-01,-1.54 \mathrm{e}-01)$ & $8.14 \mathrm{e}-01$ & $\mathcal{Z}_{2}$ & 8 & $\mathcal{Z}_{0}$ \\
\hline 2.5 .5 & $(0.195,0.981)$ & $(-8.75 \mathrm{e}-01,1.20 \mathrm{e}-01)$ & $8.83 \mathrm{e}-01$ & $\mathcal{Z}_{2}$ & 4 & $\mathcal{Z}_{0}$ \\
\hline 2.5 .6 & $(0.000,1.000)$ & $(-9.05 \mathrm{e}-01,-5.77 \mathrm{e}-02)$ & $9.06 \mathrm{e}-01$ & $\mathcal{Z}_{2}$ & 4 & $\mathcal{Z}_{0}$ \\
\hline 2.5 .7 & $(-0.981,0.195)$ & $(-9.81 \mathrm{e}-01,1.95 \mathrm{e}-01)$ & $1.00 \mathrm{e}+00$ & $\mathcal{Z}_{0}$ & 0 & $\mathcal{Z}_{0}$ \\
\hline
\end{tabular}

\begin{tabular}{|c||c||c||c||c||c||c|}
\hline \multicolumn{7}{|c|}{$\alpha=\pi / 32=0.09817 \quad \Rightarrow \quad{ }^{2}$} \\
\hline$\#$ & $\left(\dot{x}_{0}, \dot{y}_{0}\right)$ & $\left(\dot{x}_{f}, \dot{y}_{f}\right)$ & $\left\|\mathbf{v}_{f}\right\|$ & $\mathcal{Z}\left(\mathbf{v}_{0}\right)$ & $N$ & Stop \\
\hline 2.6 .1 & $(1.000,0.000)$ & $(-9.50 \mathrm{e}-01,-0.00 \mathrm{e}+00)$ & $9.50 \mathrm{e}-01$ & $\mathcal{Z}_{12}$ & 1 & $\mathcal{Z}_{0}$ \\
2.6 .2 & $(0.999,0.033)$ & $(-6.70 \mathrm{e}-01,-1.43 \mathrm{e}-02)$ & $6.70 \mathrm{e}-01$ & $\mathcal{Z}_{12}$ & 14 & $\mathcal{Z}_{0}$ \\
2.6 .3 & $(0.998,0.066)$ & $(-6.61 \mathrm{e}-01,-3.65 \mathrm{e}-02)$ & $6.62 \mathrm{e}-01$ & $\mathcal{Z}_{12}$ & 15 & $\mathcal{Z}_{0}$ \\
2.6 .4 & $(0.995,0.098)$ & $(-6.59 \mathrm{e}-01,-4.75 \mathrm{e}-02)$ & $6.61 \mathrm{e}-01$ & $\mathcal{Z}_{2}$ & 16 & $\mathcal{Z}_{0}$ \\
2.6 .5 & $(0.098,0.995)$ & $(-8.06 \mathrm{e}-01,-1.62 \mathrm{e}-02)$ & $8.06 \mathrm{e}-01$ & $\mathcal{Z}_{2}$ & 8 & $\mathcal{Z}_{0}$ \\
2.6 .6 & $(0.000,1.000)$ & $(-8.25 \mathrm{e}-01,-6.41 \mathrm{e}-02)$ & $8.27 \mathrm{e}-01$ & $\mathcal{Z}_{2}$ & 7 & $\mathcal{Z}_{0}$ \\
2.6 .7 & $(-0.995,0.098)$ & $(-9.95 \mathrm{e}-01,9.80 \mathrm{e}-02)$ & $1.00 \mathrm{e}+00$ & $\mathcal{Z}_{0}$ & 0 & $\mathcal{Z}_{0}$ \\
\hline
\end{tabular}

\begin{tabular}{|c|c|c|c|c|c|c|}
\hline \multicolumn{3}{|c|}{$\alpha=\pi / 64=0.04909$} & \multicolumn{4}{|c|}{$k=0.04913$} \\
\hline \# & $\left(\dot{x}_{0}, \dot{y}_{0}\right)$ & $\left(\dot{x}_{f}, \dot{y}_{f}\right)$ & $\left\|\mathbf{v}_{f}\right\|$ & $\mathcal{Z}\left(\mathbf{v}_{0}\right)$ & $N$ & Stop \\
\hline$\overline{2.7 .1}$ & $(1.000,0.000$ & $(-9.50 \mathrm{e}-01,-0.00 \mathrm{e}+00)$ & $9.50 \mathrm{e}-01$ & $\mathcal{Z}_{12}$ & 1 & $\mathcal{Z}_{0}$ \\
\hline 2.7 .2 & $(1.000,0.016$ & $(-4.29 \mathrm{e}-01,7.51 \mathrm{e}-03)$ & $4.29 \mathrm{e}-01$ & $\mathcal{Z}_{12}$ & 31 & $\mathcal{Z}_{0}$ \\
\hline 2.7 .3 & $(0.999,0.033)$ & $(-4.27 \mathrm{e}-01,1.20 \mathrm{e}-02)$ & $4.28 \mathrm{e}-01$ & $\mathcal{Z}_{12}$ & 32 & $\mathcal{Z}_{0}$ \\
\hline 2.7 .4 & $(0.999,0.049)$ & $(-4.27 \mathrm{e}-01,1.49 \mathrm{e}-02)$ & $4.27 \mathrm{e}-01$ & $\mathcal{Z}_{2}$ & 33 & $\mathcal{Z}_{0}$ \\
\hline 2.7 .5 & $(0.049,0.999)$ & $(-6.84 \mathrm{e}-01,1.95 \mathrm{e}-02)$ & $6.84 \mathrm{e}-01$ & $\mathcal{Z}_{2}$ & 14 & $\mathcal{Z}_{0}$ \\
\hline 2.7 .6 & $(0.000,1.000$ & $(-7.02 \mathrm{e}-01,-1.50 \mathrm{e}-02)$ & $7.02 \mathrm{e}-01$ & $\mathcal{Z}_{2}$ & 14 & $\mathcal{Z}_{0}$ \\
\hline 2.7 .7 & $(-0.999,0.049)$ & $(-9.99 \mathrm{e}-01,4.91 \mathrm{e}-02)$ & $1.00 \mathrm{e}+00$ & $\mathcal{Z}_{0}$ & 0 & $\mathcal{Z}_{0}$ \\
\hline
\end{tabular}


Non ideal case: $\varepsilon=0.75$.

\begin{tabular}{|c|c|c|c|c|c|c|}
\hline \multicolumn{7}{|c|}{$\alpha=\pi / 4=0.78540$} \\
\hline \# & $\left(\dot{x}_{0}, \dot{y}_{0}\right)$ & $\left(\dot{x}_{f}, \dot{y}_{f}\right)$ & $\left\|\mathbf{v}_{f}\right\|$ & $\mathcal{Z}\left(\mathbf{v}_{0}\right)$ & $N$ & Stop \\
\hline 3.1 .1 & $(1.000,0.000)$ & $(-7.50 \mathrm{e}-01,-0.00 \mathrm{e}+00)$ & $7.50 \mathrm{e}-01$ & $\mathcal{Z}_{12}$ & 1 & $\mathcal{Z}_{0}$ \\
\hline 3.1 .2 & $(0.949,0.316)$ & $(-7.12 \mathrm{e}-01,-2.37 \mathrm{e}-01)$ & $7.50 \mathrm{e}-01$ & $\mathcal{Z}_{12}$ & 1 & $\mathcal{Z}_{0}$ \\
\hline 3.1 .3 & $(0.832,0.555)$ & $(-6.24 \mathrm{e}-01,-4.16 \mathrm{e}-01)$ & $7.50 \mathrm{e}-01$ & $\mathcal{Z}_{12}$ & 1 & $\mathcal{Z}_{0}$ \\
\hline 3.1 .4 & $(0.707,0.707)$ & $(-5.30 \mathrm{e}-01,-5.30 \mathrm{e}-01)$ & $7.50 \mathrm{e}-01$ & $\mathcal{Z}_{2}$ & 1 & $\mathcal{Z}_{0}$ \\
\hline 3.1 .5 & $(0.707,0.707)$ & $(-5.30 \mathrm{e}-01,-5.30 \mathrm{e}-01)$ & $7.50 \mathrm{e}-01$ & $\mathcal{Z}_{2}$ & 1 & $\mathcal{Z}_{0}$ \\
\hline 3.1 .6 & $(0.000,1.000)$ & $(-8.75 \mathrm{e}-01,1.25 \mathrm{e}-01)$ & $8.84 \mathrm{e}-01$ & $\mathcal{Z}_{2}$ & 1 & $\mathcal{Z}_{0}$ \\
\hline 3.1 .7 & $(-0.707,0.707)$ & $(-7.07 \mathrm{e}-01,7.07 \mathrm{e}-01)$ & $1.00 \mathrm{e}+00$ & $\mathcal{Z}_{0}$ & 0 & $\mathcal{Z}_{0}$ \\
\hline
\end{tabular}

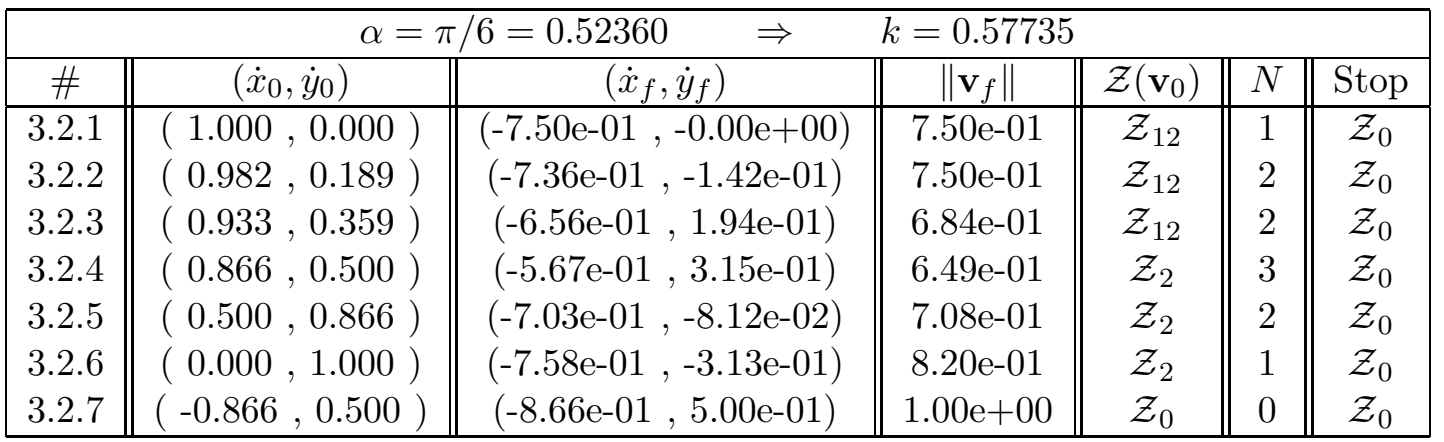

\begin{tabular}{|c||c||c||c||c||c||c|}
\hline \multicolumn{7}{|c|}{$\alpha=\pi / 8=0.39270 \quad \Rightarrow \quad k=0.41421$} \\
\hline$\#$ & $\left(\dot{x}_{0}, \dot{y}_{0}\right)$ & $\left(\dot{x}_{f}, \dot{y}_{f}\right)$ & $\left\|\mathbf{v}_{f}\right\|$ & $\mathcal{Z}\left(\mathbf{v}_{0}\right)$ & $N$ & Stop \\
\hline 3.3 .1 & $(1.000,0.000)$ & $(-7.50 \mathrm{e}-01,-0.00 \mathrm{e}+00)$ & $7.50 \mathrm{e}-01$ & $\mathcal{Z}_{12}$ & 1 & $\mathcal{Z}_{0}$ \\
3.3 .2 & $(0.991,0.137)$ & $(-6.46 \mathrm{e}-01,2.48 \mathrm{e}-01)$ & $6.92 \mathrm{e}-01$ & $\mathcal{Z}_{12}$ & 3 & $\mathcal{Z}_{0}$ \\
3.3 .3 & $(0.964,0.266)$ & $(-5.84 \mathrm{e}-01,-1.04 \mathrm{e}-01)$ & $5.93 \mathrm{e}-01$ & $\mathcal{Z}_{12}$ & 3 & $\mathcal{Z}_{0}$ \\
3.3 .4 & $(0.924,0.383)$ & $(-5.24 \mathrm{e}-01,-1.95 \mathrm{e}-01)$ & $5.59 \mathrm{e}-01$ & $\mathcal{Z}_{2}$ & 4 & $\mathcal{Z}_{0}$ \\
3.3 .5 & $(0.383,0.924)$ & $(-6.42 \mathrm{e}-01,1.65 \mathrm{e}-01)$ & $6.63 \mathrm{e}-01$ & $\mathcal{Z}_{2}$ & 2 & $\mathcal{Z}_{0}$ \\
3.3 .6 & $(0.000,1.000)$ & $(-7.66 \mathrm{e}-01,-1.39 \mathrm{e}-01)$ & $7.78 \mathrm{e}-01$ & $\mathcal{Z}_{2}$ & 2 & $\mathcal{Z}_{0}$ \\
3.3 .7 & $(-0.924,0.383)$ & $(-9.24 \mathrm{e}-01,3.83 \mathrm{e}-01)$ & $1.00 \mathrm{e}+00$ & $\mathcal{Z}_{0}$ & 0 & $\mathcal{Z}_{0}$ \\
\hline
\end{tabular}




\begin{tabular}{|c||c||c||c||c||c||c|}
\hline \multicolumn{7}{|c|}{$\alpha=\pi / 12=0.26180 \quad \Rightarrow \quad k=0.26795$} \\
\hline$\#$ & $\left(\dot{x}_{0}, \dot{y}_{0}\right)$ & $\left(\dot{x}_{f}, \dot{y}_{f}\right)$ & $\left\|\mathbf{v}_{f}\right\|$ & $\mathcal{Z}\left(\mathbf{v}_{0}\right)$ & $N$ & Stop \\
\hline 3.4 .1 & $(1.000,0.000)$ & $(-7.50 \mathrm{e}-01,-0.00 \mathrm{e}+00)$ & $7.50 \mathrm{e}-01$ & $\mathcal{Z}_{12}$ & 1 & $\mathcal{Z}_{0}$ \\
3.4 .2 & $(0.996,0.089)$ & $(-5.26 \mathrm{e}-01,2.44 \mathrm{e}-02)$ & $5.26 \mathrm{e}-01$ & $\mathcal{Z}_{12}$ & 4 & $\mathcal{Z}_{0}$ \\
3.4 .3 & $(0.984,0.176)$ & $(-4.22 \mathrm{e}-01,-6.60 \mathrm{e}-02)$ & $4.27 \mathrm{e}-01$ & $\mathcal{Z}_{12}$ & 5 & $\mathcal{Z}_{0}$ \\
3.4 .4 & $(0.966,0.259)$ & $(-4.04 \mathrm{e}-01,-6.57 \mathrm{e}-02)$ & $4.10 \mathrm{e}-01$ & $\mathcal{Z}_{2}$ & 6 & $\mathcal{Z}_{0}$ \\
3.4 .5 & $(0.259,0.966)$ & $(-5.94 \mathrm{e}-01,-2.27 \mathrm{e}-02)$ & $5.95 \mathrm{e}-01$ & $\mathcal{Z}_{2}$ & 3 & $\mathcal{Z}_{0}$ \\
3.4 .6 & $(0.000,1.000)$ & $(-6.77 \mathrm{e}-01,1.58 \mathrm{e}-01)$ & $6.95 \mathrm{e}-01$ & $\mathcal{Z}_{2}$ & 3 & $\mathcal{Z}_{0}$ \\
3.4 .7 & $(-0.966,0.259)$ & $(-9.66 \mathrm{e}-01,2.59 \mathrm{e}-01)$ & $1.00 \mathrm{e}+00$ & $\mathcal{Z}_{0}$ & 0 & $\mathcal{Z}_{0}$ \\
\hline
\end{tabular}

\begin{tabular}{|c|c|c|c|c|c|c|}
\hline \multicolumn{3}{|c|}{$\alpha=\pi / 16=0.19635$} & \multicolumn{4}{|c|}{$k=0.19891$} \\
\hline$\#$ & $\left(\dot{x}_{0}, \dot{y}_{0}\right)$ & $\left(\dot{x}_{f}, \dot{y}_{f}\right)$ & $\left\|\mathbf{v}_{f}\right\|$ & $\mathcal{Z}\left(\mathbf{v}_{0}\right)$ & $N$ & Stop \\
\hline 3.5 .1 & $(1.000,0.000$ & $(-7.50 \mathrm{e}-01,-0.00 \mathrm{e}+00)$ & $7.50 \mathrm{e}-01$ & $\mathcal{Z}_{12}$ & 1 & $\mathcal{Z}_{0}$ \\
\hline 3.5 .2 & $(0.998,0.066)$ & $(-3.69 \mathrm{e}-01,-1.44 \mathrm{e}-03)$ & $3.69 \mathrm{e}-01$ & $\mathcal{Z}_{12}$ & 6 & $\mathcal{Z}_{0}$ \\
\hline 3.5 .3 & $(0.991,0.131)$ & $(-2.97 \mathrm{e}-01,-4.66 \mathrm{e}-02)$ & $3.01 \mathrm{e}-01$ & $\mathcal{Z}_{12}$ & 8 & $\mathcal{Z}_{0}$ \\
\hline 3.5 .4 & $(0.981,0.195)$ & $(-2.92 \mathrm{e}-01,3.11 \mathrm{e}-03)$ & $2.92 \mathrm{e}-01$ & $\mathcal{Z}_{2}$ & 8 & $\mathcal{Z}_{0}$ \\
\hline 3.5 .5 & $(0.195,0.981)$ & $(-5.36 \mathrm{e}-01,-4.63 \mathrm{e}-02)$ & $5.38 \mathrm{e}-01$ & $\mathcal{Z}_{2}$ & 4 & $\mathcal{Z}_{0}$ \\
\hline 3.5 .6 & $(0.000,1.000)$ & $(-6.24 \mathrm{e}-01,-6.13 \mathrm{e}-02)$ & $6.27 \mathrm{e}-01$ & $\mathcal{Z}_{2}$ & 3 & $\mathcal{Z}_{0}$ \\
\hline 3.5.7 & $(-0.981,0.195)$ & $(-9.81 \mathrm{e}-01,1.95 \mathrm{e}-01)$ & $1.00 \mathrm{e}+00$ & $\mathcal{Z}_{0}$ & 0 & $\mathcal{Z}_{0}$ \\
\hline
\end{tabular}

\begin{tabular}{|c||c||c||c||c||c||c|}
\hline \multicolumn{7}{|c|}{$\alpha=\pi / 32=0.09817 \quad \Rightarrow \quad{ }^{2}$} \\
\hline$\#$ & $\left(\dot{x}_{0}, \dot{y}_{0}\right)$ & $\left(\dot{x}_{f}, \dot{y}_{f}\right)$ & $\left\|\mathbf{v}_{f}\right\|$ & $\mathcal{Z}\left(\mathbf{v}_{0}\right)$ & $N$ & Stop \\
\hline 3.6 .1 & $(1.000,0.000)$ & $(-7.50 \mathrm{e}-01,-0.00 \mathrm{e}+00)$ & $7.50 \mathrm{e}-01$ & $\mathcal{Z}_{12}$ & 1 & $\mathcal{Z}_{0}$ \\
3.6 .2 & $(0.999,0.033)$ & $(-3.93 \mathrm{e}-02,-1.60 \mathrm{e}-03)$ & $3.94 \mathrm{e}-02$ & $\mathcal{Z}_{12}$ & 20 & $\mathcal{Z}_{0}$ \\
3.6 .3 & $(0.998,0.066)$ & $(-3.51 \mathrm{e}-02,2.16 \mathrm{e}-03)$ & $3.52 \mathrm{e}-02$ & $\mathcal{Z}_{12}$ & 22 & $\mathcal{Z}_{0}$ \\
3.6 .4 & $(0.995,0.098)$ & $(-3.48 \mathrm{e}-02,1.36 \mathrm{e}-03)$ & $3.49 \mathrm{e}-02$ & $\mathcal{Z}_{2}$ & 23 & $\mathcal{Z}_{0}$ \\
3.6 .5 & $(0.098,0.995)$ & $(-3.85 \mathrm{e}-01,7.72 \mathrm{e}-03)$ & $3.85 \mathrm{e}-01$ & $\mathcal{Z}_{2}$ & 6 & $\mathcal{Z}_{0}$ \\
3.6 .6 & $(0.000,1.000)$ & $(-4.48 \mathrm{e}-01,-3.63 \mathrm{e}-02)$ & $4.49 \mathrm{e}-01$ & $\mathcal{Z}_{2}$ & 6 & $\mathcal{Z}_{0}$ \\
3.6 .7 & $(-0.995,0.098)$ & $(-9.95 \mathrm{e}-01,9.80 \mathrm{e}-02)$ & $1.00 \mathrm{e}+00$ & $\mathcal{Z}_{0}$ & 0 & $\mathcal{Z}_{0}$ \\
\hline
\end{tabular}

\begin{tabular}{|c||c||c||c||c||c||c|}
\hline \multicolumn{7}{|c|}{$\alpha=\pi / 64=0.04909 \quad \Rightarrow$} \\
\hline$\#$ & $\left(\dot{x}_{0}, \dot{y}_{0}\right)$ & $\left(\dot{x}_{f}, \dot{y}_{f}\right)$ & $\left\|\mathbf{v}_{f}\right\|$ & $\mathcal{Z}\left(\mathbf{v}_{0}\right)$ & $N$ & Stop \\
\hline 3.7 .1 & $(1.000,0.000)$ & $(-7.50 \mathrm{e}-01,-0.00 \mathrm{e}+00)$ & $7.50 \mathrm{e}-01$ & $\mathcal{Z}_{12}$ & 1 & $\mathcal{Z}_{0}$ \\
3.7 .2 & $(1.000,0.016)$ & $(9.00 \mathrm{e}-13,3.57 \mathrm{e}-13)$ & $9.68 \mathrm{e}-13$ & $\mathcal{Z}_{12}$ & 712 & $\|\cdot\|$ \\
3.7 .3 & $(0.999,0.033)$ & $(9.02 \mathrm{e}-13,3.58 \mathrm{e}-13)$ & $9.71 \mathrm{e}-13$ & $\mathcal{Z}_{12}$ & 714 & $\|\cdot\|$ \\
3.7 .4 & $(0.999,0.049)$ & $(9.18 \mathrm{e}-13,3.65 \mathrm{e}-13)$ & $9.88 \mathrm{e}-13$ & $\mathcal{Z}_{2}$ & 714 & $\|\cdot\|$ \\
3.7 .5 & $(0.049,0.999)$ & $(-2.41 \mathrm{e}-01,-8.67 \mathrm{e}-03)$ & $2.41 \mathrm{e}-01$ & $\mathcal{Z}_{2}$ & 9 & $\mathcal{Z}_{0}$ \\
3.7 .6 & $(0.000,1.000)$ & $(-2.82 \mathrm{e}-01,5.15 \mathrm{e}-03)$ & $2.82 \mathrm{e}-01$ & $\mathcal{Z}_{2}$ & 9 & $\mathcal{Z}_{0}$ \\
3.7 .7 & $(-0.999,0.049)$ & $(-9.99 \mathrm{e}-01,4.91 \mathrm{e}-02)$ & $1.00 \mathrm{e}+00$ & $\mathcal{Z}_{0}$ & 0 & $\mathcal{Z}_{0}$ \\
\hline
\end{tabular}


Non ideal case: $\varepsilon=0.5$.

\begin{tabular}{|c|c|c|c|c|c|c|}
\hline \multicolumn{3}{|c|}{$\alpha=\pi / 4=0.78540$} & \multicolumn{4}{|l|}{$k=1$} \\
\hline \# & $\left(\dot{x}_{0}, \dot{y}_{0}\right)$ & $\left(\dot{x}_{f}, \dot{y}_{f}\right)$ & $\left\|\mathbf{v}_{f}\right\|$ & $\mathcal{Z}\left(\mathbf{v}_{0}\right)$ & $N$ & Stop \\
\hline 4.1 .1 & $(1.000,0.000)$ & $(-5.00 \mathrm{e}-01,-0.00 \mathrm{e}+00)$ & $5.00 \mathrm{e}-01$ & $\mathcal{Z}_{12}$ & 1 & $\mathcal{Z}_{0}$ \\
\hline 4.1 .2 & $(0.949,0.316)$ & $(-4.74 \mathrm{e}-01,-1.58 \mathrm{e}-01)$ & $5.00 \mathrm{e}-01$ & $\mathcal{Z}_{12}$ & 1 & $\mathcal{Z}_{0}$ \\
\hline 4.1 .3 & $(0.832,0.555)$ & $(-4.16 \mathrm{e}-01,-2.77 \mathrm{e}-01)$ & $5.00 \mathrm{e}-01$ & $\mathcal{Z}_{12}$ & 1 & $\mathcal{Z}_{0}$ \\
\hline 4.1.4 & $(0.707,0.707)$ & $(-3.54 \mathrm{e}-01,-3.54 \mathrm{e}-01)$ & $5.00 \mathrm{e}-01$ & $\mathcal{Z}_{2}$ & 1 & $\mathcal{Z}_{0}$ \\
\hline 4.1 .5 & $(0.707,0.707)$ & $(-3.54 \mathrm{e}-01,-3.54 \mathrm{e}-01)$ & $5.00 \mathrm{e}-01$ & $\mathcal{Z}_{2}$ & 1 & $\mathcal{Z}_{0}$ \\
\hline 4.1 .6 & $(0.000,1.000)$ & $(-7.50 \mathrm{e}-01,2.50 \mathrm{e}-01)$ & $7.91 \mathrm{e}-01$ & $\mathcal{Z}_{2}$ & 1 & $\mathcal{Z}_{0}$ \\
\hline 4.1.7 & $(-0.707,0.707)$ & $(-7.07 \mathrm{e}-01,7.07 \mathrm{e}-01)$ & $1.00 \mathrm{e}+00$ & $\mathcal{Z}_{0}$ & 0 & $\mathcal{Z}_{0}$ \\
\hline
\end{tabular}

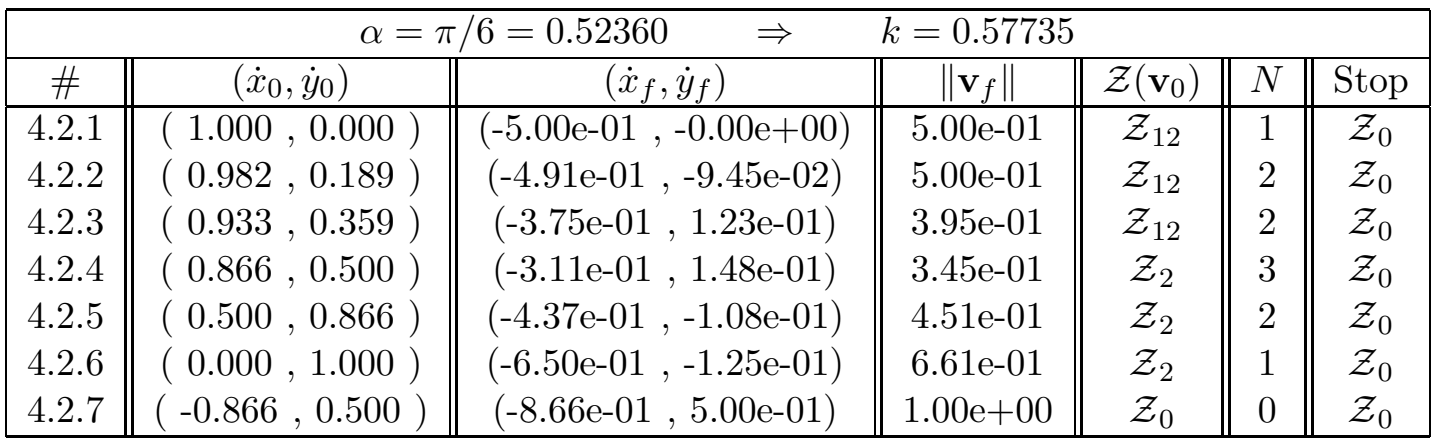

\begin{tabular}{|c||c||c||c||c||c||c|}
\hline \multicolumn{7}{|c|}{$\alpha=\pi / 8=0.39270 \quad \Rightarrow \quad k=0.41421$} \\
\hline$\#$ & $\left(\dot{x}_{0}, \dot{y}_{0}\right)$ & $\left(\dot{x}_{f}, \dot{y}_{f}\right)$ & $\left\|\mathbf{v}_{f}\right\|$ & $\mathcal{Z}\left(\mathbf{v}_{0}\right)$ & $N$ & Stop \\
\hline 4.3 .1 & $(1.000,0.000)$ & $(-5.00 \mathrm{e}-01,-0.00 \mathrm{e}+00)$ & $5.00 \mathrm{e}-01$ & $\mathcal{Z}_{12}$ & 1 & $\mathcal{Z}_{0}$ \\
4.3 .2 & $(0.991,0.137)$ & $(-3.80 \mathrm{e}-01,1.42 \mathrm{e}-01)$ & $4.06 \mathrm{e}-01$ & $\mathcal{Z}_{12}$ & 3 & $\mathcal{Z}_{0}$ \\
4.3 .3 & $(0.964,0.266)$ & $(-2.52 \mathrm{e}-01,-7.92 \mathrm{e}-02)$ & $2.64 \mathrm{e}-01$ & $\mathcal{Z}_{12}$ & 3 & $\mathcal{Z}_{0}$ \\
4.3 .4 & $(0.924,0.383)$ & $(-2.20 \mathrm{e}-01,-4.04 \mathrm{e}-02)$ & $2.24 \mathrm{e}-01$ & $\mathcal{Z}_{2}$ & 4 & $\mathcal{Z}_{0}$ \\
4.3 .5 & $(0.383,0.924)$ & $(-3.94 \mathrm{e}-01,2.80 \mathrm{e}-02)$ & $3.95 \mathrm{e}-01$ & $\mathcal{Z}_{2}$ & 2 & $\mathcal{Z}_{0}$ \\
4.3 .6 & $(0.000,1.000)$ & $(-5.62 \mathrm{e}-01,-2.03 \mathrm{e}-01)$ & $5.98 \mathrm{e}-01$ & $\mathcal{Z}_{2}$ & 2 & $\mathcal{Z}_{0}$ \\
4.3 .7 & $(-0.924,0.383)$ & $(-9.24 \mathrm{e}-01,3.83 \mathrm{e}-01)$ & $1.00 \mathrm{e}+00$ & $\mathcal{Z}_{0}$ & 0 & $\mathcal{Z}_{0}$ \\
\hline
\end{tabular}




\begin{tabular}{|c||c||c||c||c||c||c|}
\hline \multicolumn{7}{|c|}{$\alpha=\pi / 12=0.26180 \quad \Rightarrow \quad k=0.26795$} \\
\hline$\#$ & $\left(\dot{x}_{0}, \dot{y}_{0}\right)$ & $\left(\dot{x}_{f}, \dot{y}_{f}\right)$ & $\left\|\mathbf{v}_{f}\right\|$ & $\mathcal{Z}\left(\mathbf{v}_{0}\right)$ & $N$ & Stop \\
\hline 4.4 .1 & $(1.000,0.000)$ & $(-5.00 \mathrm{e}-01,-0.00 \mathrm{e}+00)$ & $5.00 \mathrm{e}-01$ & $\mathcal{Z}_{12}$ & 1 & $\mathcal{Z}_{0}$ \\
4.4 .2 & $(0.996,0.089)$ & $(-1.77 \mathrm{e}-01,2.70 \mathrm{e}-02)$ & $1.79 \mathrm{e}-01$ & $\mathcal{Z}_{12}$ & 4 & $\mathcal{Z}_{0}$ \\
4.4 .3 & $(0.984,0.176)$ & $(-7.68 \mathrm{e}-02,1.96 \mathrm{e}-02)$ & $7.93 \mathrm{e}-02$ & $\mathcal{Z}_{12}$ & 7 & $\mathcal{Z}_{0}$ \\
4.4 .4 & $(0.966,0.259)$ & $(-6.83 \mathrm{e}-02,-5.65 \mathrm{e}-03)$ & $6.85 \mathrm{e}-02$ & $\mathcal{Z}_{2}$ & 7 & $\mathcal{Z}_{0}$ \\
4.4 .5 & $(0.259,0.966)$ & $(-3.22 \mathrm{e}-01,5.39 \mathrm{e}-02)$ & $3.26 \mathrm{e}-01$ & $\mathcal{Z}_{2}$ & 3 & $\mathcal{Z}_{0}$ \\
4.4 .6 & $(0.000,1.000)$ & $(-4.87 \mathrm{e}-01,1.90 \mathrm{e}-02)$ & $4.88 \mathrm{e}-01$ & $\mathcal{Z}_{2}$ & 2 & $\mathcal{Z}_{0}$ \\
4.4 .7 & $(-0.966,0.259)$ & $(-9.66 \mathrm{e}-01,2.59 \mathrm{e}-01)$ & $1.00 \mathrm{e}+00$ & $\mathcal{Z}_{0}$ & 0 & $\mathcal{Z}_{0}$ \\
\hline
\end{tabular}

\begin{tabular}{|c|c|c|c|c|c|c|}
\hline \multicolumn{7}{|c|}{$\alpha=\pi / 16=0.19635$} \\
\hline \# & $\left(\dot{x}_{0}, \dot{y}_{0}\right)$ & $\left(\dot{x}_{f}, \dot{y}_{f}\right)$ & $\mid \mathbf{v}_{f} \|$ & $\mathcal{Z}\left(\mathbf{v}_{0}\right)$ & $N$ & Stop \\
\hline 4.5 .1 & $(1.000,0.000)$ & $(-5.00 \mathrm{e}-01,-0.00 \mathrm{e}+00)$ & $5.00 \mathrm{e}-01$ & $\mathcal{Z}_{12}$ & 1 & $\mathcal{Z}_{0}$ \\
\hline 4.5.2 & $(0.998,0.066)$ & $(-2.37 \mathrm{e}-02,3.83 \mathrm{e}-03)$ & $2.40 \mathrm{e}-02$ & $\mathcal{Z}_{12}$ & 9 & $\mathcal{Z}_{0}$ \\
\hline 4.5.3 & $(0.991,0.131)$ & $(-4.87 \mathrm{e}-03,4.16 \mathrm{e}-04)$ & $4.89 \mathrm{e}-03$ & $\mathcal{Z}_{12}$ & 14 & $\mathcal{Z}_{0}$ \\
\hline 4.5.4 & $(0.981,0.195)$ & $(-4.41 \mathrm{e}-03,-1.20 \mathrm{e}-04)$ & $4.41 \mathrm{e}-03$ & $\mathcal{Z}_{2}$ & 15 & $\mathcal{Z}_{0}$ \\
\hline 4.5.5 & $(0.195,0.981)$ & $(-2.74 \mathrm{e}-01,-1.70 \mathrm{e}-02)$ & $2.74 \mathrm{e}-01$ & $\mathcal{Z}_{2}$ & 3 & $\mathcal{Z}_{0}$ \\
\hline 4.5.6 & $(0.000,1.000)$ & $(-4.08 \mathrm{e}-01,6.38 \mathrm{e}-0$ & $4.13 \mathrm{e}-01$ & $\mathcal{Z}_{2}$ & 3 & $\mathcal{Z}_{0}$ \\
\hline 4.5.7 & $(-0.981,0.195)$ & $(-9.81 \mathrm{e}-01,1.95 \mathrm{e}-01)$ & $1.00 \mathrm{e}+00$ & $\mathcal{Z}_{0}$ & 0 & $\mathcal{Z}_{0}$ \\
\hline
\end{tabular}

\begin{tabular}{|c||c||c||c||c||c||c|}
\hline \multicolumn{7}{|c|}{$\alpha=\pi / 32=0.09817 \quad \Rightarrow$} \\
\hline$\#$ & $\left(\dot{x}_{0}, \dot{y}_{0}\right)$ & $\left(\dot{x}_{f}, \dot{y}_{f}\right)$ & $\left\|\mathbf{v}_{f}\right\|$ & $\mathcal{Z}\left(\mathbf{v}_{0}\right)$ & $N$ & Stop \\
\hline 4.6 .1 & $(1.000,0.000)$ & $(-5.00 \mathrm{e}-01,-0.00 \mathrm{e}+00)$ & $5.00 \mathrm{e}-01$ & $\mathcal{Z}_{12}$ & 1 & $\mathcal{Z}_{0}$ \\
4.6 .2 & $(0.999,0.033)$ & $(9.17 \mathrm{e}-13,-2.96 \mathrm{e}-13)$ & $9.64 \mathrm{e}-13$ & $\mathcal{Z}_{12}$ & 433 & $\|\cdot\|$ \\
4.6 .3 & $(0.998,0.066)$ & $(9.05 \mathrm{e}-13,2.93 \mathrm{e}-13)$ & $9.51 \mathrm{e}-13$ & $\mathcal{Z}_{12}$ & 436 & $\|\cdot\|$ \\
4.6 .4 & $(0.995,0.098)$ & $(9.39 \mathrm{e}-13,3.03 \mathrm{e}-13)$ & $9.86 \mathrm{e}-13$ & $\mathcal{Z}_{2}$ & 436 & $\|\cdot\|$ \\
4.6 .5 & $(0.098,0.995)$ & $(-1.67 \mathrm{e}-01,1.25 \mathrm{e}-02)$ & $1.67 \mathrm{e}-01$ & $\mathcal{Z}_{2}$ & 5 & $\mathcal{Z}_{0}$ \\
4.6 .6 & $(0.000,1.000)$ & $(-2.51 \mathrm{e}-01,-3.75 \mathrm{e}-04)$ & $2.51 \mathrm{e}-01$ & $\mathcal{Z}_{2}$ & 4 & $\mathcal{Z}_{0}$ \\
4.6 .7 & $(-0.995,0.098)$ & $(-9.95 \mathrm{e}-01,9.80 \mathrm{e}-02)$ & $1.00 \mathrm{e}+00$ & $\mathcal{Z}_{0}$ & 0 & $\mathcal{Z}_{0}$ \\
\hline
\end{tabular}

\begin{tabular}{|c||c||c||c||c||c||c|}
\hline \multicolumn{7}{|c|}{$\alpha=\pi / 64=0.04909 \quad \Rightarrow$} \\
\hline$\#$ & $\left(\dot{x}_{0}, \dot{y}_{0}\right)$ & $\left(\dot{x}_{f}, \dot{y}_{f}\right)$ & $\left\|\mathbf{v}_{f}\right\|$ & $\mathcal{Z}\left(\mathbf{v}_{0}\right)$ & $N$ & Stop \\
\hline 4.7 .1 & $(1.000,0.000)$ & $(-5.00 \mathrm{e}-01,-0.00 \mathrm{e}+00)$ & $5.00 \mathrm{e}-01$ & $\mathcal{Z}_{12}$ & 1 & $\mathcal{Z}_{0}$ \\
4.7 .2 & $(1.000,0.016)$ & $(9.87 \mathrm{e}-13,-1.48 \mathrm{e}-13)$ & $9.98 \mathrm{e}-13$ & $\mathcal{Z}_{12}$ & 1869 & $\|\cdot\|$ \\
4.7 .3 & $(0.999,0.033)$ & $(9.83 \mathrm{e}-13,1.48 \mathrm{e}-13)$ & $9.94 \mathrm{e}-13$ & $\mathcal{Z}_{12}$ & 1872 & $\|\cdot\|$ \\
4.7 .4 & $(0.999,0.049)$ & $(9.76 \mathrm{e}-13,-1.47 \mathrm{e}-13)$ & $9.87 \mathrm{e}-13$ & $\mathcal{Z}_{2}$ & 1873 & $\|\cdot\|$ \\
4.7 .5 & $(0.049,0.999)$ & $(-9.21 \mathrm{e}-02,3.09 \mathrm{e}-03)$ & $9.21 \mathrm{e}-02$ & $\mathcal{Z}_{2}$ & 6 & $\mathcal{Z}_{0}$ \\
4.7 .6 & $(0.000,1.000)$ & $(-1.38 \mathrm{e}-01,-3.70 \mathrm{e}-03)$ & $1.38 \mathrm{e}-01$ & $\mathcal{Z}_{2}$ & 6 & $\mathcal{Z}_{0}$ \\
4.7 .7 & $(-0.999,0.049)$ & $(-9.99 \mathrm{e}-01,4.91 \mathrm{e}-02)$ & $1.00 \mathrm{e}+00$ & $\mathcal{Z}_{0}$ & 0 & $\mathcal{Z}_{0}$ \\
\hline
\end{tabular}


Non ideal case: $\varepsilon=0.25$.

\begin{tabular}{|c|c|c|c|c|c|c|}
\hline \multicolumn{7}{|c|}{$\alpha=\pi / 4=0.78540$} \\
\hline \# & $\left(\dot{x}_{0}, \dot{y}_{0}\right)$ & $\left(\dot{x}_{f}, \dot{y}_{f}\right)$ & $\left\|\mathbf{v}_{f}\right\|$ & $\mathcal{Z}\left(\mathbf{v}_{0}\right)$ & $N$ & Stop \\
\hline 5.1 .1 & $(1.000,0.000)$ & $(-2.50 \mathrm{e}-01,-0.00 \mathrm{e}+00)$ & $2.50 \mathrm{e}-01$ & $\mathcal{Z}_{12}$ & 1 & $\mathcal{Z}_{0}$ \\
\hline 5.1 .2 & $(0.949,0.316)$ & $(-2.37 \mathrm{e}-01,-7.91 \mathrm{e}-02)$ & $2.50 \mathrm{e}-01$ & $\mathcal{Z}_{12}$ & 1 & $\mathcal{Z}_{0}$ \\
\hline 5.1 .3 & $(0.832,0.555)$ & $(-2.08 \mathrm{e}-01,-1.39 \mathrm{e}-01)$ & $2.50 \mathrm{e}-01$ & $\mathcal{Z}_{12}$ & 1 & $\mathcal{Z}_{0}$ \\
\hline 5.1 .4 & $(0.707,0.707)$ & $(-1.77 \mathrm{e}-01,-1.77 \mathrm{e}-01)$ & $2.50 \mathrm{e}-01$ & $\mathcal{Z}_{2}$ & 1 & $\mathcal{Z}_{0}$ \\
\hline 5.1 .5 & $(0.707,0.707)$ & $(-1.77 \mathrm{e}-01,-1.77 \mathrm{e}-01)$ & $2.50 \mathrm{e}-01$ & $\mathcal{Z}_{2}$ & 1 & $\mathcal{Z}_{0}$ \\
\hline 5.1 .6 & $(0.000,1.000)$ & $(-6.25 \mathrm{e}-01,3.75 \mathrm{e}-01)$ & $7.29 \mathrm{e}-01$ & $\mathcal{Z}_{2}$ & 1 & $\mathcal{Z}_{0}$ \\
\hline 5.1 .7 & $(-0.707,0.707)$ & $(-7.07 \mathrm{e}-01,7.07 \mathrm{e}-01)$ & $1.00 \mathrm{e}+00$ & $\mathcal{Z}_{0}$ & 0 & $\mathcal{Z}_{0}$ \\
\hline
\end{tabular}

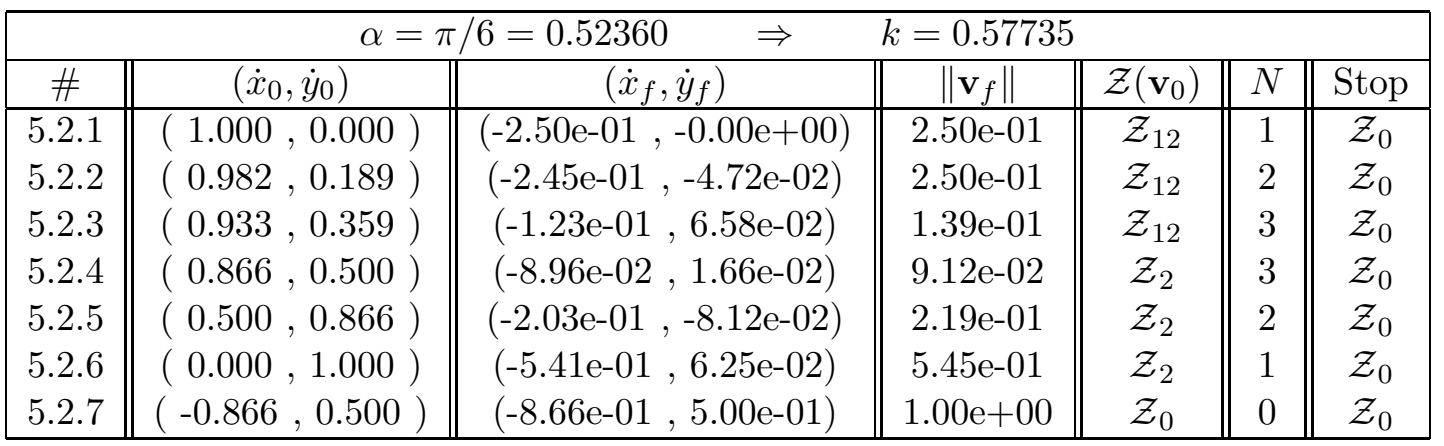

\begin{tabular}{|c||c||c||c||c||c||c|}
\hline \multicolumn{7}{|c|}{$\alpha=\pi / 8=0.39270 \quad \Rightarrow \quad k=0.41421$} \\
\hline$\#$ & $\left(\dot{x}_{0}, \dot{y}_{0}\right)$ & $\left(\dot{x}_{f}, \dot{y}_{f}\right)$ & $\left\|\mathbf{v}_{f}\right\|$ & $\mathcal{Z}\left(\mathbf{v}_{0}\right)$ & $N$ & Stop \\
\hline 5.3 .1 & $(1.000,0.000)$ & $(-2.50 \mathrm{e}-01,-0.00 \mathrm{e}+00)$ & $2.50 \mathrm{e}-01$ & $\mathcal{Z}_{12}$ & 1 & $\mathcal{Z}_{0}$ \\
5.3 .2 & $(0.991,0.137)$ & $(-1.36 \mathrm{e}-01,4.14 \mathrm{e}-02)$ & $1.42 \mathrm{e}-01$ & $\mathcal{Z}_{12}$ & 3 & $\mathcal{Z}_{0}$ \\
5.3 .3 & $(0.964,0.266)$ & $(-2.28 \mathrm{e}-02,7.07 \mathrm{e}-03)$ & $2.39 \mathrm{e}-02$ & $\mathcal{Z}_{12}$ & 5 & $\mathcal{Z}_{0}$ \\
5.3 .4 & $(0.924,0.383)$ & $(-1.15 \mathrm{e}-02,-1.95 \mathrm{e}-03)$ & $1.16 \mathrm{e}-02$ & $\mathcal{Z}_{2}$ & 6 & $\mathcal{Z}_{0}$ \\
5.3 .5 & $(0.383,0.924)$ & $(-1.80 \mathrm{e}-01,-2.68 \mathrm{e}-02)$ & $1.82 \mathrm{e}-01$ & $\mathcal{Z}_{2}$ & 2 & $\mathcal{Z}_{0}$ \\
5.3 .6 & $(0.000,1.000)$ & $(-4.42 \mathrm{e}-01,-6.69 \mathrm{e}-02)$ & $4.47 \mathrm{e}-01$ & $\mathcal{Z}_{2}$ & 1 & $\mathcal{Z}_{0}$ \\
5.3 .7 & $(-0.924,0.383)$ & $(-9.24 \mathrm{e}-01,3.83 \mathrm{e}-01)$ & $1.00 \mathrm{e}+00$ & $\mathcal{Z}_{0}$ & 0 & $\mathcal{Z}_{0}$ \\
\hline
\end{tabular}




\begin{tabular}{|c||c||c||c||c||c||c|}
\hline \multicolumn{7}{|c|}{$\alpha=\pi / 12=0.26180 \quad \Rightarrow \quad \|=0.26795$} \\
\hline$\#$ & $\left(\dot{x}_{0}, \dot{y}_{0}\right)$ & $\left(\dot{x}_{f}, \dot{y}_{f}\right)$ & $\left\|\mathbf{v}_{f}\right\|$ & $\mathcal{Z}\left(\mathbf{v}_{0}\right)$ & $N$ & Stop \\
\hline 5.4 .1 & $(1.000,0.000)$ & $(-2.50 \mathrm{e}-01,-0.00 \mathrm{e}+00)$ & $2.50 \mathrm{e}-01$ & $\mathcal{Z}_{12}$ & 1 & $\mathcal{Z}_{0}$ \\
5.4 .2 & $(0.996,0.089)$ & $(6.96 \mathrm{e}-13,3.73 \mathrm{e}-13)$ & $7.90 \mathrm{e}-13$ & $\mathcal{Z}_{12}$ & 92 & $\|\cdot\|$ \\
5.4 .3 & $(0.984,0.176)$ & $(7.93 \mathrm{e}-13,4.25 \mathrm{e}-13)$ & $9.00 \mathrm{e}-13$ & $\mathcal{Z}_{12}$ & 96 & $\|\cdot\|$ \\
5.4 .4 & $(0.966,0.259)$ & $(7.32 \mathrm{e}-13,-3.93 \mathrm{e}-13)$ & $8.31 \mathrm{e}-13$ & $\mathcal{Z}_{2}$ & 97 & $\|\cdot\|$ \\
5.4 .5 & $(0.259,0.966)$ & $(-1.35 \mathrm{e}-01,1.99 \mathrm{e}-02)$ & $1.36 \mathrm{e}-01$ & $\mathcal{Z}_{2}$ & 2 & $\mathcal{Z}_{0}$ \\
5.4 .6 & $(0.000,1.000)$ & $(-3.38 \mathrm{e}-01,-7.00 \mathrm{e}-02)$ & $3.45 \mathrm{e}-01$ & $\mathcal{Z}_{2}$ & 2 & $\mathcal{Z}_{0}$ \\
5.4 .7 & $(-0.966,0.259)$ & $(-9.66 \mathrm{e}-01,2.59 \mathrm{e}-01)$ & $1.00 \mathrm{e}+00$ & $\mathcal{Z}_{0}$ & 0 & $\mathcal{Z}_{0}$ \\
\hline
\end{tabular}

\begin{tabular}{|c|c|c|c|c|c|c|}
\hline \multicolumn{7}{|c|}{$\alpha=\pi / 16=0.19635$} \\
\hline$\#$ & $\left(\dot{x}_{0}, \dot{y}_{0}\right)$ & $\left(\dot{x}_{f}, \dot{y}_{f}\right)$ & $\mid \mathbf{v}_{f} \|$ & $\overline{\mathcal{Z}\left(\mathbf{v}_{0}\right)}$ & $N$ & Stop \\
\hline 5.5 .1 & $(1.000,0.000)$ & $(-2.50 \mathrm{e}-01,-0.00 \mathrm{e}+00)$ & $2.50 \mathrm{e}-01$ & $\mathcal{Z}_{12}$ & 1 & $\mathcal{Z}_{0}$ \\
\hline 5.5 .2 & $(0.998,0.066)$ & $(8.25 \mathrm{e}-13,2.97 \mathrm{e}-13)$ & $8.77 \mathrm{e}-13$ & $\mathcal{Z}_{12}$ & 190 & $\|\cdot\|$ \\
\hline 5.5 .3 & $(0.991,0.131)$ & $(8.76 \mathrm{e}-13,-3.16 \mathrm{e}-13)$ & $9.31 \mathrm{e}-13$ & $\mathcal{Z}_{12}$ & 193 & $\cdot \|$ \\
\hline 5.5 .4 & $(0.981,0.195)$ & $(8.36 \mathrm{e}-13,3.01 \mathrm{e}-13)$ & $8.89 \mathrm{e}-13$ & $\mathcal{Z}_{2}$ & 194 & $\|\cdot\|$ \\
\hline 5.5 .5 & $(0.195,0.981)$ & $(-1.09 \mathrm{e}-01,1.75 \mathrm{e}-02)$ & $1.11 \mathrm{e}-01$ & $\mathcal{Z}_{2}$ & 3 & $\mathcal{Z}_{0}$ \\
\hline 5.5 .6 & $(0.000,1.000)$ & $(-2.76 \mathrm{e}-01,-1.62 \mathrm{e}-02)$ & $2.77 \mathrm{e}-01$ & $\mathcal{Z}_{2}$ & 2 & $\mathcal{Z}_{0}$ \\
\hline 5.5 .7 & $(-0.981,0.195)$ & $(-9.81 \mathrm{e}-01,1.95 \mathrm{e}-01)$ & $1.00 \mathrm{e}+00$ & $\mathcal{Z}_{0}$ & 0 & $\mathcal{Z}_{0}$ \\
\hline
\end{tabular}

\begin{tabular}{|c||c||c||c||c||c||c|}
\hline \multicolumn{9}{|c|}{$\alpha=\pi / 32=0.09817 \quad \Rightarrow \quad \dot{Z}^{\prime}$} & \multicolumn{1}{l|}{$k=0.09849$} \\
\hline$\#$ & $\left(\dot{x}_{0}, \dot{y}_{0}\right)$ & $\left(\dot{x}_{f}, \dot{y}_{f}\right)$ & $\|$ & $\mathbf{v}_{f} \|$ & Stop \\
\hline 5.6 .1 & $(1.000,0.000)$ & $(-2.50 \mathrm{e}-01,-0.00 \mathrm{e}+00)$ & $2.50 \mathrm{e}-01$ & $\mathcal{Z}_{12}$ & 1 & $\mathcal{Z}_{0}$ \\
5.6 .2 & $(0.999,0.033)$ & $(9.84 \mathrm{e}-13,1.64 \mathrm{e}-13)$ & $9.98 \mathrm{e}-13$ & $\mathcal{Z}_{12}$ & 836 & $\|\cdot\|$ \\
5.6 .3 & $(0.998,0.066)$ & $(9.63 \mathrm{e}-13,1.61 \mathrm{e}-13)$ & $9.76 \mathrm{e}-13$ & $\mathcal{Z}_{12}$ & 840 & $\|\cdot\|$ \\
5.6 .4 & $(0.995,0.098)$ & $(9.84 \mathrm{e}-13,1.64 \mathrm{e}-13)$ & $9.97 \mathrm{e}-13$ & $\mathcal{Z}_{2}$ & 840 & $\|\cdot\|$ \\
5.6 .5 & $(0.098,0.995)$ & $(-6.08 \mathrm{e}-02,-5.81 \mathrm{e}-03)$ & $6.11 \mathrm{e}-02$ & $\mathcal{Z}_{2}$ & 4 & $\mathcal{Z}_{0}$ \\
5.6 .6 & $(0.000,1.000)$ & $(-1.53 \mathrm{e}-01,8.29 \mathrm{e}-03)$ & $1.53 \mathrm{e}-01$ & $\mathcal{Z}_{2}$ & 3 & $\mathcal{Z}_{0}$ \\
5.6 .7 & $(-0.995,0.098)$ & $(-9.95 \mathrm{e}-01,9.80 \mathrm{e}-02)$ & $1.00 \mathrm{e}+00$ & $\mathcal{Z}_{0}$ & 0 & $\mathcal{Z}_{0}$ \\
\hline
\end{tabular}

\begin{tabular}{|c||c||c||c||c||c||c|}
\hline \multicolumn{7}{|c|}{$\alpha=\pi / 64=0.04909 \quad \Rightarrow$} \\
\hline$\#$ & $\left(\dot{x}_{0}, \dot{y}_{0}\right)$ & $\left(\dot{x}_{f}, \dot{y}_{f}\right)$ & $\left\|\mathbf{v}_{f}\right\|$ & $\mathcal{Z}\left(\mathbf{v}_{0}\right)$ & $N$ & Stop \\
\hline 5.7 .1 & $(1.000,0.000)$ & $(-2.50 \mathrm{e}-01,-0.00 \mathrm{e}+00)$ & $2.50 \mathrm{e}-01$ & $\mathcal{Z}_{12}$ & 1 & $\mathcal{Z}_{0}$ \\
5.7 .2 & $(1.000,0.016)$ & $(9.92 \mathrm{e}-13,-8.15 \mathrm{e}-14)$ & $9.95 \mathrm{e}-13$ & $\mathcal{Z}_{12}$ & 3417 & $\|\cdot\|$ \\
5.7 .3 & $(0.999,0.033)$ & $(9.94 \mathrm{e}-13,8.18 \mathrm{e}-14)$ & $9.98 \mathrm{e}-13$ & $\mathcal{Z}_{12}$ & 3420 & $\|\cdot\|$ \\
5.7 .4 & $(0.999,0.049)$ & $(9.92 \mathrm{e}-13,-8.16 \mathrm{e}-14)$ & $9.95 \mathrm{e}-13$ & $\mathcal{Z}_{2}$ & 3421 & $\|\cdot\|$ \\
5.7 .5 & $(0.049,0.999)$ & $(-3.19 \mathrm{e}-02,1.39 \mathrm{e}-03)$ & $3.19 \mathrm{e}-02$ & $\mathcal{Z}_{2}$ & 4 & $\mathcal{Z}_{0}$ \\
5.7 .6 & $(0.000,1.000)$ & $(-7.98 \mathrm{e}-02,-2.53 \mathrm{e}-03)$ & $7.99 \mathrm{e}-02$ & $\mathcal{Z}_{2}$ & 4 & $\mathcal{Z}_{0}$ \\
5.7 .7 & $(-0.999,0.049)$ & $(-9.99 \mathrm{e}-01,4.91 \mathrm{e}-02)$ & $1.00 \mathrm{e}+00$ & $\mathcal{Z}_{0}$ & 0 & $\mathcal{Z}_{0}$ \\
\hline
\end{tabular}


Non ideal case: $\varepsilon=0.05$.

\begin{tabular}{|c|c|c|c|c|c|c|}
\hline \multicolumn{7}{|c|}{$\alpha=\pi / 4=0.78540$} \\
\hline \# & $\left(\dot{x}_{0}, \dot{y}_{0}\right)$ & $\left(\dot{x}_{f}, \dot{y}_{f}\right)$ & $\left\|\mathbf{v}_{f}\right\|$ & $\mathcal{Z}\left(\mathbf{v}_{0}\right)$ & $N$ & Stop \\
\hline 6.1 .1 & $(1.000,0.000)$ & $(-5.00 \mathrm{e}-02,-0.00 \mathrm{e}+00)$ & $5.00 \mathrm{e}-02$ & $\mathcal{Z}_{12}$ & 1 & $\mathcal{Z}_{0}$ \\
\hline 6.1 .2 & $(0.949,0.316)$ & $(-4.74 \mathrm{e}-02,-1.58 \mathrm{e}-02)$ & $5.00 \mathrm{e}-02$ & $\mathcal{Z}_{12}$ & 1 & $\mathcal{Z}_{0}$ \\
\hline 6.1 .3 & $(0.832,0.555)$ & $(-4.16 \mathrm{e}-02,-2.77 \mathrm{e}-02)$ & $5.00 \mathrm{e}-02$ & $\mathcal{Z}_{12}$ & 1 & $\mathcal{Z}_{0}$ \\
\hline 6.1 .4 & $(0.707,0.707)$ & $(-3.54 \mathrm{e}-02,-3.54 \mathrm{e}-02)$ & $5.00 \mathrm{e}-02$ & $\mathcal{Z}_{2}$ & 1 & $\mathcal{Z}_{0}$ \\
\hline 6.1 .5 & $(0.707,0.707)$ & $(-3.54 \mathrm{e}-02,-3.54 \mathrm{e}-02)$ & $5.00 \mathrm{e}-02$ & $\mathcal{Z}_{2}$ & 1 & $\mathcal{Z}_{0}$ \\
\hline 6.1 .6 & $(0.000,1.000)$ & $(-5.25 \mathrm{e}-01,4.75 \mathrm{e}-01)$ & $7.08 \mathrm{e}-01$ & $\mathcal{Z}_{2}$ & 1 & $\mathcal{Z}_{0}$ \\
\hline 6.1 .7 & $(-0.707,0.707)$ & $(-7.07 \mathrm{e}-01,7.07 \mathrm{e}-01)$ & $1.00 \mathrm{e}+00$ & $\mathcal{Z}_{0}$ & 0 & $\mathcal{Z}_{0}$ \\
\hline
\end{tabular}

\begin{tabular}{|c||c||c||c||c||c||c|}
\hline \multicolumn{7}{|c|}{$\alpha=\pi / 6=0.52360 \quad \Rightarrow$} \\
\hline$\#$ & $\left(\dot{x}_{0}, \dot{y}_{0}\right)$ & $\left(\dot{x}_{f}, \dot{y}_{f}\right)$ & $\left\|\mathbf{v}_{f}\right\|$ & $\mathcal{Z}\left(\mathbf{v}_{0}\right)$ & $N$ & Stop \\
\hline 6.2 .1 & $(1.000,0.000)$ & $(-5.00 \mathrm{e}-02,-0.00 \mathrm{e}+00)$ & $5.00 \mathrm{e}-02$ & $\mathcal{Z}_{12}$ & 1 & $\mathcal{Z}_{0}$ \\
6.2 .2 & $(0.982,0.189)$ & $(-4.91 \mathrm{e}-02,-9.45 \mathrm{e}-03)$ & $5.00 \mathrm{e}-02$ & $\mathcal{Z}_{12}$ & 2 & $\mathcal{Z}_{0}$ \\
6.2 .3 & $(0.933,0.359)$ & $(4.71 \mathrm{e}-13,3.49 \mathrm{e}-13)$ & $5.86 \mathrm{e}-13$ & $\mathcal{Z}_{12}$ & 30 & $\|\cdot\|$ \\
6.2 .4 & $(0.866,0.500)$ & $(5.08 \mathrm{e}-13,-3.77 \mathrm{e}-13)$ & $6.33 \mathrm{e}-13$ & $\mathcal{Z}_{2}$ & 31 & $\|\cdot\|$ \\
6.2 .5 & $(0.500,0.866)$ & $(-3.81 \mathrm{e}-02,-2.06 \mathrm{e}-02)$ & $4.33 \mathrm{e}-02$ & $\mathcal{Z}_{2}$ & 2 & $\mathcal{Z}_{0}$ \\
6.2 .6 & $(0.000,1.000)$ & $(-4.55 \mathrm{e}-01,2.12 \mathrm{e}-01)$ & $5.02 \mathrm{e}-01$ & $\mathcal{Z}_{2}$ & 1 & $\mathcal{Z}_{0}$ \\
6.2 .7 & $(-0.866,0.500)$ & $(-8.66 \mathrm{e}-01,5.00 \mathrm{e}-01)$ & $1.00 \mathrm{e}+00$ & $\mathcal{Z}_{0}$ & 0 & $\mathcal{Z}_{0}$ \\
\hline
\end{tabular}

\begin{tabular}{|c|c|c|c|c|c|c|}
\hline \multicolumn{7}{|c|}{$\alpha=\pi / 8=0.39270$} \\
\hline \# & $\left(\dot{x}_{0}, \dot{y}_{0}\right)$ & $\left(\dot{x}_{f}, \dot{y}_{f}\right)$ & $\left\|\mathbf{v}_{f}\right\|$ & $\mathcal{Z}\left(\mathbf{v}_{0}\right)$ & $N$ & Stop \\
\hline 6.3 .1 & $(1.000,0.000$ & $(-5.00 \mathrm{e}-02,-0.00 \mathrm{e}+00)$ & $5.00 \mathrm{e}-02$ & $\mathcal{Z}_{12}$ & 1 & $\mathcal{Z}_{0}$ \\
\hline 6.3 .2 & $(0.991,0.137)$ & $(6.35 \mathrm{e}-13,-3.06 \mathrm{e}-13)$ & $7.04 \mathrm{e}-13$ & $\mathcal{Z}_{12}$ & 65 & $\|\cdot\|$ \\
\hline 6.3 .3 & $(0.964,0.266)$ & $(8.37 \mathrm{e}-13,4.03 \mathrm{e}-13)$ & $9.29 \mathrm{e}-13$ & $\mathcal{Z}_{12}$ & 68 & $\|\cdot\|$ \\
\hline 6.3 .4 & $(0.924,0.383)$ & $(7.47 \mathrm{e}-13,-3.60 \mathrm{e}-13)$ & $8.29 \mathrm{e}-13$ & $\mathcal{Z}_{2}$ & 69 & $\|\cdot\|$ \\
\hline 6.3 .5 & $(0.383,0.924)$ & $(-3.33 \mathrm{e}-02,-1.19 \mathrm{e}-02)$ & $3.54 \mathrm{e}-02$ & $\mathcal{Z}_{2}$ & 2 & $\mathcal{Z}_{0}$ \\
\hline 6.3 .6 & $(0.000,1.000)$ & $(-3.71 \mathrm{e}-01,1.04 \mathrm{e}-01)$ & $3.85 \mathrm{e}-01$ & $\mathcal{Z}_{2}$ & 1 & $\mathcal{Z}_{0}$ \\
\hline 6.3 .7 & $(-0.924,0.383)$ & $(-9.24 \mathrm{e}-01,3.83 \mathrm{e}-01)$ & $1.00 \mathrm{e}+00$ & $\mathcal{Z}_{0}$ & 0 & $\mathcal{Z}_{0}$ \\
\hline
\end{tabular}




\begin{tabular}{|c||c||c||c||c||c||c|}
\hline \multicolumn{7}{|c|}{$\alpha=\pi / 12=0.26180 \quad \Rightarrow$} \\
\hline$\#$ & $\left(\dot{x}_{0}, \dot{y}_{0}\right)$ & $\left(\dot{x}_{f}, \dot{y}_{f}\right)$ & $\left\|\mathbf{v}_{f}\right\|$ & $\mathcal{Z}\left(\mathbf{v}_{0}\right)$ & $N$ & Stop \\
\hline 6.4 .1 & $(1.000,0.000)$ & $(-5.00 \mathrm{e}-02,-0.00 \mathrm{e}+00)$ & $5.00 \mathrm{e}-02$ & $\mathcal{Z}_{12}$ & 1 & $\mathcal{Z}_{0}$ \\
6.4 .2 & $(0.996,0.089)$ & $(9.29 \mathrm{e}-13,-2.80 \mathrm{e}-13)$ & $9.70 \mathrm{e}-13$ & $\mathcal{Z}_{12}$ & 167 & $\|\cdot\|$ \\
6.4 .3 & $(0.984,0.176)$ & $(8.28 \mathrm{e}-13,-2.50 \mathrm{e}-13)$ & $8.65 \mathrm{e}-13$ & $\mathcal{Z}_{12}$ & 171 & $\|\cdot\|$ \\
6.4 .4 & $(0.966,0.259)$ & $(9.28 \mathrm{e}-13,-2.80 \mathrm{e}-13)$ & $9.69 \mathrm{e}-13$ & $\mathcal{Z}_{2}$ & 171 & $\|\cdot\|$ \\
6.4 .5 & $(0.259,0.966)$ & $(-2.47 \mathrm{e}-02,-4.38 \mathrm{e}-03)$ & $2.51 \mathrm{e}-02$ & $\mathcal{Z}_{2}$ & 2 & $\mathcal{Z}_{0}$ \\
6.4 .6 & $(0.000,1.000)$ & $(-2.63 \mathrm{e}-01,2.03 \mathrm{e}-02)$ & $2.63 \mathrm{e}-01$ & $\mathcal{Z}_{2}$ & 1 & $\mathcal{Z}_{0}$ \\
6.4 .7 & $(-0.966,0.259)$ & $(-9.66 \mathrm{e}-01,2.59 \mathrm{e}-01)$ & $1.00 \mathrm{e}+00$ & $\mathcal{Z}_{0}$ & 0 & $\mathcal{Z}_{0}$ \\
\hline
\end{tabular}

\begin{tabular}{|c||c||c||c||c||c||c|}
\hline \multicolumn{7}{|c|}{$\alpha=\pi / 16=0.19635 \quad \Rightarrow$} \\
\hline$\#$ & $\left(\dot{x}_{0}, \dot{y}_{0}\right)$ & $\left(\dot{x}_{f}, \dot{y}_{f}\right)$ & $\left\|\mathbf{v}_{f}\right\|$ & $\mathcal{Z}\left(\mathbf{v}_{0}\right)$ & $N$ & Stop \\
\hline 6.5 .1 & $(1.000,0.000)$ & $(-5.00 \mathrm{e}-02,-0.00 \mathrm{e}+00)$ & $5.00 \mathrm{e}-02$ & $\mathcal{Z}_{12}$ & 1 & $\mathcal{Z}_{0}$ \\
6.5 .2 & $(0.998,0.066)$ & $(9.54 \mathrm{e}-13,-2.12 \mathrm{e}-13)$ & $9.77 \mathrm{e}-13$ & $\mathcal{Z}_{12}$ & 309 & $\|\cdot\|$ \\
6.5 .3 & $(0.991,0.131)$ & $(9.10 \mathrm{e}-13,-2.02 \mathrm{e}-13)$ & $9.33 \mathrm{e}-13$ & $\mathcal{Z}_{12}$ & 313 & $\|\cdot\|$ \\
6.5 .4 & $(0.981,0.195)$ & $(9.69 \mathrm{e}-13,-2.15 \mathrm{e}-13)$ & $9.93 \mathrm{e}-13$ & $\mathcal{Z}_{2}$ & 313 & $\|\cdot\|$ \\
6.5 .5 & $(0.195,0.981)$ & $(-1.92 \mathrm{e}-02,-1.47 \mathrm{e}-03)$ & $1.93 \mathrm{e}-02$ & $\mathcal{Z}_{2}$ & 2 & $\mathcal{Z}_{0}$ \\
6.5 .6 & $(0.000,1.000)$ & $(-2.01 \mathrm{e}-01,-1.00 \mathrm{e}-02)$ & $2.01 \mathrm{e}-01$ & $\mathcal{Z}_{2}$ & 1 & $\mathcal{Z}_{0}$ \\
6.5 .7 & $(-0.981,0.195)$ & $(-9.81 \mathrm{e}-01,1.95 \mathrm{e}-01)$ & $1.00 \mathrm{e}+00$ & $\mathcal{Z}_{0}$ & 0 & $\mathcal{Z}_{0}$ \\
\hline
\end{tabular}

\begin{tabular}{|c|c|c|c|c|c|c|}
\hline \multicolumn{3}{|c|}{$\alpha=\pi / 32=0.09817$} & \multicolumn{4}{|c|}{$k=0.09849$} \\
\hline \# & $\left(\dot{x}_{0}, \dot{y}_{0}\right)$ & $\left(\dot{x}_{f}, \dot{y}_{f}\right)$ & $\left\|\mathbf{v}_{f}\right\|$ & $\overline{\mathcal{Z}\left(\mathbf{v}_{0}\right)}$ & $N$ & Stop \\
\hline 6.6 .1 & $(1.000,0.000)$ & $(-5.00 \mathrm{e}-02,-0.00 \mathrm{e}+00)$ & $5.00 \mathrm{e}-02$ & $\mathcal{Z}_{12}$ & 1 & $\mathcal{Z}_{0}$ \\
\hline 6.6 .2 & $(0.999,0.033)$ & $(9.80 \mathrm{e}-13,1.07 \mathrm{e}-13)$ & $9.85 \mathrm{e}-13$ & $\mathcal{Z}_{12}$ & 1282 & $\|\cdot\|$ \\
\hline 6.6 .3 & $(0.998,0.066)$ & $(9.75 \mathrm{e}-13,1.06 \mathrm{e}-13)$ & $9.81 \mathrm{e}-13$ & $\mathcal{Z}_{12}$ & 1286 & $\|\cdot\|$ \\
\hline 6.6 .4 & $(0.995,0.098)$ & $(9.90 \mathrm{e}-13,1.08 \mathrm{e}-13)$ & $9.96 \mathrm{e}-13$ & $\mathcal{Z}_{2}$ & 1286 & $\|\cdot\|$ \\
\hline 6.6 .5 & $(0.098,0.995)$ & $(-1.00 \mathrm{e}-02,9.60 \mathrm{e}-04)$ & $1.00 \mathrm{e}-02$ & $\mathcal{Z}_{2}$ & 3 & $\mathcal{Z}_{0}$ \\
\hline 6.6 .6 & $(0.000,1.000)$ & $(-1.05 \mathrm{e}-01,-8.90 \mathrm{e}-03)$ & $1.06 \mathrm{e}-01$ & $\mathcal{Z}_{2}$ & 2 & $\mathcal{Z}_{0}$ \\
\hline 6.6 .7 & $(-0.995,0.098)$ & $(-9.95 \mathrm{e}-01,9.80 \mathrm{e}-02)$ & $1.00 \mathrm{e}+00$ & $\mathcal{Z}_{0}$ & 0 & $\mathcal{Z}_{0}$ \\
\hline
\end{tabular}

\begin{tabular}{|c||c||c||c||c||c||c|}
\hline \multicolumn{7}{|c|}{$\alpha=\pi / 64=0.04909 \quad \Rightarrow$} \\
\hline$\#$ & $\left(\dot{x}_{0}, \dot{y}_{0}\right)$ & $\left(\dot{x}_{f}, \dot{y}_{f}\right)$ & $\left\|\mathbf{v}_{f}\right\|$ & $\mathcal{Z}\left(\mathbf{v}_{0}\right)$ & $N$ & Stop \\
\hline 6.7 .1 & $(1.000,0.000)$ & $(-5.00 \mathrm{e}-02,-0.00 \mathrm{e}+00)$ & $5.00 \mathrm{e}-02$ & $\mathcal{Z}_{12}$ & 1 & $\mathcal{Z}_{0}$ \\
6.7 .2 & $(1.000,0.016)$ & $(9.98 \mathrm{e}-13,5.42 \mathrm{e}-14)$ & $1.00 \mathrm{e}-12$ & $\mathcal{Z}_{12}$ & 5172 & $\|\cdot\|$ \\
6.7 .3 & $(0.999,0.033)$ & $(9.97 \mathrm{e}-13,5.42 \mathrm{e}-14)$ & $9.99 \mathrm{e}-13$ & $\mathcal{Z}_{12}$ & 5176 & $\|\cdot\|$ \\
6.7 .4 & $(0.999,0.049)$ & $(9.96 \mathrm{e}-13,-5.41 \mathrm{e}-14)$ & $9.97 \mathrm{e}-13$ & $\mathcal{Z}_{2}$ & 5177 & $\|\cdot\|$ \\
6.7 .5 & $(0.049,0.999)$ & $(-5.12 \mathrm{e}-03,1.52 \mathrm{e}-04)$ & $5.12 \mathrm{e}-03$ & $\mathcal{Z}_{2}$ & 3 & $\mathcal{Z}_{0}$ \\
6.7 .6 & $(0.000,1.000)$ & $(-5.38 \mathrm{e}-02,-3.94 \mathrm{e}-04)$ & $5.38 \mathrm{e}-02$ & $\mathcal{Z}_{2}$ & 2 & $\mathcal{Z}_{0}$ \\
6.7 .7 & $(-0.999,0.049)$ & $(-9.99 \mathrm{e}-01,4.91 \mathrm{e}-02)$ & $1.00 \mathrm{e}+00$ & $\mathcal{Z}_{0}$ & 0 & $\mathcal{Z}_{0}$ \\
\hline
\end{tabular}


Non ideal case: $\varepsilon=0$.

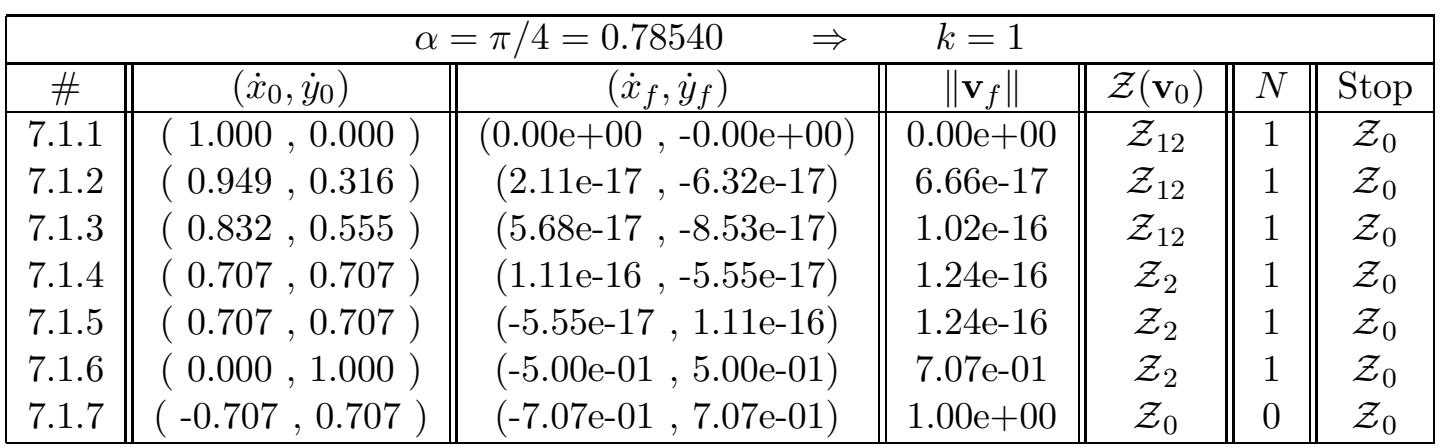

\begin{tabular}{|c||c||c||c||c||c||c|}
\hline \multicolumn{7}{|c|}{$\alpha=\pi / 6=0.52360 \quad \Rightarrow \quad k=0.57735$} \\
\hline$\#$ & $\left(\dot{x}_{0}, \dot{y}_{0}\right)$ & $\left(\dot{x}_{f}, \dot{y}_{f}\right)$ & $\left\|\mathbf{v}_{f}\right\|$ & $\mathcal{Z}\left(\mathbf{v}_{0}\right)$ & $N$ & Stop \\
\hline 7.2 .1 & $(1.000,0.000)$ & $(0.00 \mathrm{e}+00,-0.00 \mathrm{e}+00)$ & $0.00 \mathrm{e}+00$ & $\mathcal{Z}_{12}$ & 1 & $\mathcal{Z}_{0}$ \\
7.2 .2 & $(0.982,0.189)$ & $(0.00 \mathrm{e}+00,0.00 \mathrm{e}+00)$ & $0.00 \mathrm{e}+00$ & $\mathcal{Z}_{12}$ & 2 & $\mathcal{Z}_{0}$ \\
7.2 .3 & $(0.933,0.359)$ & $(4.85 \mathrm{e}-13,2.80 \mathrm{e}-13)$ & $5.60 \mathrm{e}-13$ & $\mathcal{Z}_{12}$ & 40 & $\|\cdot\|$ \\
7.2 .4 & $(0.866,0.500)$ & $(7.88 \mathrm{e}-13,4.55 \mathrm{e}-13)$ & $9.09 \mathrm{e}-13$ & $\mathcal{Z}_{2}$ & 40 & $\|\cdot\|$ \\
7.2 .5 & $(0.500,0.866)$ & $(0.00 \mathrm{e}+00,0.00 \mathrm{e}+00)$ & $0.00 \mathrm{e}+00$ & $\mathcal{Z}_{2}$ & 1 & $\mathcal{Z}_{0}$ \\
7.2 .6 & $(0.000,1.000)$ & $(-4.33 \mathrm{e}-01,2.50 \mathrm{e}-01)$ & $5.00 \mathrm{e}-01$ & $\mathcal{Z}_{2}$ & 1 & $\mathcal{Z}_{0}$ \\
7.2 .7 & $(-0.866,0.500)$ & $(-8.66 \mathrm{e}-01,5.00 \mathrm{e}-01)$ & $1.00 \mathrm{e}+00$ & $\mathcal{Z}_{0}$ & 0 & $\mathcal{Z}_{0}$ \\
\hline
\end{tabular}

\begin{tabular}{|c|c|c|c|c|c|c|}
\hline \multicolumn{7}{|c|}{$\alpha=\pi / 8=0.39270$} \\
\hline \# & $\left(\dot{x}_{0}, \dot{y}_{0}\right)$ & $\left(\dot{x}_{f}, \dot{y}_{f}\right)$ & $\left|\mathbf{v}_{f}\right| \mid$ & $\mathcal{Z}\left(\mathbf{v}_{0}\right)$ & $N$ & Stop \\
\hline 7.3 .1 & $(1.000,0.000)$ & $(0.00 \mathrm{e}+00,-0.00 \mathrm{e}+00)$ & $0.00 \mathrm{e}+00$ & $\mathcal{Z}_{12}$ & 1 & $\overline{\mathcal{Z}_{0}}$ \\
\hline 7.3 .2 & $(0.991,0.137)$ & $(6.87 \mathrm{e}-13,-2.85 \mathrm{e}-13)$ & $7.44 \mathrm{e}-13$ & $\mathcal{Z}_{12}$ & 77 & $\|\cdot\|$ \\
\hline 7.3.3 & $(0.964,0.266)$ & $(6.64 \mathrm{e}-13,2.75 \mathrm{e}-13)$ & $7.19 \mathrm{e}-13$ & $\mathcal{Z}_{12}$ & 80 & $\|\cdot\|$ \\
\hline 7.3.4 & $(0.924,0.383)$ & $(8.40 \mathrm{e}-13,3.48 \mathrm{e}-13)$ & $9.09 \mathrm{e}-13$ & $\mathcal{Z}_{2}$ & 80 & $\|\cdot\|$ \\
\hline 7.3 .5 & $(0.383,0.924)$ & $(4.74 \mathrm{e}-17,0.00 \mathrm{e}+00)$ & $4.74 \mathrm{e}-17$ & $\mathcal{Z}_{2}$ & 1 & $\mathcal{Z}_{0}$ \\
\hline 7.3.6 & $(0.000,1.000)$ & $(-3.54 \mathrm{e}-01,1.46 \mathrm{e}-01)$ & $3.83 \mathrm{e}-01$ & $\mathcal{Z}_{2}$ & 1 & $\mathcal{Z}_{0}$ \\
\hline 7.3.7 & $(-0.924,0.383)$ & $(-9.24 \mathrm{e}-01,3.83 \mathrm{e}-01)$ & $1.00 \mathrm{e}+00$ & $\mathcal{Z}_{0}$ & 0 & $\mathcal{Z}_{0}$ \\
\hline
\end{tabular}




\begin{tabular}{|c||c||c||c||c||c||c|}
\hline \multicolumn{7}{|c|}{$\alpha=\pi / 12=0.26180 \quad \Rightarrow$} \\
\hline$\#$ & $\left(\dot{x}_{0}, \dot{y}_{0}\right)$ & $\left(\dot{x}_{f}, \dot{y}_{f}\right)$ & $\left\|\mathbf{v}_{f}\right\|$ & $\mathcal{Z}\left(\mathbf{v}_{0}\right)$ & $N$ & Stop \\
\hline 7.4 .1 & $(1.000,0.000)$ & $(0.00 \mathrm{e}+00,-0.00 \mathrm{e}+00)$ & $0.00 \mathrm{e}+00$ & $\mathcal{Z}_{12}$ & 1 & $\mathcal{Z}_{0}$ \\
7.4 .2 & $(0.996,0.089)$ & $(8.55 \mathrm{e}-13,-2.29 \mathrm{e}-13)$ & $8.86 \mathrm{e}-13$ & $\mathcal{Z}_{12}$ & 189 & $\|\cdot\|$ \\
7.4 .3 & $(0.984,0.176)$ & $(8.85 \mathrm{e}-13,2.37 \mathrm{e}-13)$ & $9.16 \mathrm{e}-13$ & $\mathcal{Z}_{12}$ & 192 & $\|\cdot\|$ \\
7.4 .4 & $(0.966,0.259)$ & $(8.48 \mathrm{e}-13,-2.27 \mathrm{e}-13)$ & $8.78 \mathrm{e}-13$ & $\mathcal{Z}_{2}$ & 193 & $\|\cdot\|$ \\
7.4 .5 & $(0.259,0.966)$ & $(0.00 \mathrm{e}+00,0.00 \mathrm{e}+00)$ & $0.00 \mathrm{e}+00$ & $\mathcal{Z}_{2}$ & 1 & $\mathcal{Z}_{0}$ \\
7.4 .6 & $(0.000,1.000)$ & $(-2.50 \mathrm{e}-01,6.70 \mathrm{e}-02)$ & $2.59 \mathrm{e}-01$ & $\mathcal{Z}_{2}$ & 1 & $\mathcal{Z}_{0}$ \\
7.4 .7 & $(-0.966,0.259)$ & $(-9.66 \mathrm{e}-01,2.59 \mathrm{e}-01)$ & $1.00 \mathrm{e}+00$ & $\mathcal{Z}_{0}$ & 0 & $\mathcal{Z}_{0}$ \\
\hline
\end{tabular}

\begin{tabular}{|c||c||c||c||c||c||c|}
\hline \multicolumn{7}{|c|}{$\alpha=\pi / 16=0.19635 \quad \Rightarrow$} \\
\hline$\#$ & $\left(\dot{x}_{0}, \dot{y}_{0}\right)$ & $\left(\dot{x}_{f}, \dot{y}_{f}\right)$ & $\left\|\mathbf{v}_{f}\right\|$ & $\mathcal{Z}\left(\mathbf{v}_{0}\right)$ & $N$ & Stop \\
\hline 7.5 .1 & $(1.000,0.000)$ & $(0.00 \mathrm{e}+00,-0.00 \mathrm{e}+00)$ & $0.00 \mathrm{e}+00$ & $\mathcal{Z}_{12}$ & 1 & $\mathcal{Z}_{0}$ \\
7.5 .2 & $(0.998,0.066)$ & $(9.63 \mathrm{e}-13,-1.92 \mathrm{e}-13)$ & $9.82 \mathrm{e}-13$ & $\mathcal{Z}_{12}$ & 345 & $\|\cdot\|$ \\
7.5 .3 & $(0.991,0.131)$ & $(9.26 \mathrm{e}-13,-1.84 \mathrm{e}-13)$ & $9.44 \mathrm{e}-13$ & $\mathcal{Z}_{12}$ & 349 & $\|\cdot\|$ \\
7.5 .4 & $(0.981,0.195)$ & $(9.80 \mathrm{e}-13,-1.95 \mathrm{e}-13)$ & $9.99 \mathrm{e}-13$ & $\mathcal{Z}_{2}$ & 349 & $\|\cdot\|$ \\
7.5 .5 & $(0.195,0.981)$ & $(0.00 \mathrm{e}+00,0.00 \mathrm{e}+00)$ & $0.00 \mathrm{e}+00$ & $\mathcal{Z}_{2}$ & 1 & $\mathcal{Z}_{0}$ \\
7.5 .6 & $(0.000,1.000)$ & $(-1.91 \mathrm{e}-01,3.81 \mathrm{e}-02)$ & $1.95 \mathrm{e}-01$ & $\mathcal{Z}_{2}$ & 1 & $\mathcal{Z}_{0}$ \\
7.5 .7 & $(-0.981,0.195)$ & $(-9.81 \mathrm{e}-01,1.95 \mathrm{e}-01)$ & $1.00 \mathrm{e}+00$ & $\mathcal{Z}_{0}$ & 0 & $\mathcal{Z}_{0}$ \\
\hline
\end{tabular}

\begin{tabular}{|c|c|c|c|c|c|c|}
\hline \multicolumn{7}{|c|}{$\alpha=\pi / 32=0.09817$} \\
\hline \# & $\left(\dot{x}_{0}, \dot{y}_{0}\right)$ & $\left(\dot{x}_{f}, \dot{y}_{f}\right)$ & $\left\|\mathbf{v}_{f}\right\|$ & $\mathcal{Z}\left(\mathbf{v}_{0}\right)$ & $N$ & Stop \\
\hline 7.6 .1 & $(1.000,0.000)$ & $(0.00 \mathrm{e}+00,-0.00 \mathrm{e}+00)$ & $0.00 \mathrm{e}+00$ & $\mathcal{Z}_{12}$ & 1 & $\mathcal{Z}_{0}$ \\
\hline 7.6 .2 & $(0.999,0.033)$ & $(9.86 \mathrm{e}-13,9.71 \mathrm{e}-14)$ & $9.91 \mathrm{e}-13$ & $\mathcal{Z}_{12}$ & 1420 & $\|\cdot\|$ \\
\hline 7.6 .3 & $(0.998,0.066)$ & $(9.84 \mathrm{e}-13,9.69 \mathrm{e}-14)$ & $9.89 \mathrm{e}-13$ & $\mathcal{Z}_{12}$ & 1424 & $\|\cdot\|$ \\
\hline 7.6 .4 & $(0.995,0.098)$ & $(9.79 \mathrm{e}-13,-9.64 \mathrm{e}-14)$ & $9.84 \mathrm{e}-13$ & $\mathcal{Z}_{2}$ & 1425 & $\|\cdot\|$ \\
\hline 7.6 .5 & $(0.098,0.995)$ & $(-1.37 \mathrm{e}-17,0.00 \mathrm{e}+00)$ & $1.37 \mathrm{e}-17$ & $\mathcal{Z}_{2}$ & 1 & $\mathcal{Z}_{0}$ \\
\hline 7.6 .6 & $(0.000,1.000)$ & $(-9.75 \mathrm{e}-02,9.61 \mathrm{e}-03)$ & $9.80 \mathrm{e}-02$ & $\mathcal{Z}_{2}$ & 1 & $\mathcal{Z}_{0}$ \\
\hline 7.6.7 & $(-0.995,0.098)$ & $(-9.95 \mathrm{e}-01,9.80 \mathrm{e}-02)$ & $1.00 \mathrm{e}+00$ & $\mathcal{Z}_{0}$ & 0 & $\mathcal{Z}_{0}$ \\
\hline
\end{tabular}

\begin{tabular}{|c||c||c||c||c||c||c|}
\hline \multicolumn{7}{|c|}{$\alpha=\pi / 64=0.04909 \quad \| \quad 0.04913$} \\
\hline$\#$ & $\left(\dot{x}_{0}, \dot{y}_{0}\right)$ & $\left(\dot{x}_{f}, \dot{y}_{f}\right)$ & $\left\|\mathbf{v}_{f}\right\|$ & $\mathcal{Z}\left(\mathbf{v}_{0}\right)$ & $N$ & Stop \\
\hline 7.7 .1 & $(1.000,0.000)$ & $(0.00 \mathrm{e}+00,-0.00 \mathrm{e}+00)$ & $0.00 \mathrm{e}+00$ & $\mathcal{Z}_{12}$ & 1 & $\mathcal{Z}_{0}$ \\
7.7 .2 & $(1.000,0.016)$ & $(9.97 \mathrm{e}-13,4.90 \mathrm{e}-14)$ & $9.98 \mathrm{e}-13$ & $\mathcal{Z}_{12}$ & 5720 & $\|\cdot\|$ \\
7.7 .3 & $(0.999,0.033)$ & $(9.97 \mathrm{e}-13,4.90 \mathrm{e}-14)$ & $9.98 \mathrm{e}-13$ & $\mathcal{Z}_{12}$ & 5724 & $\|\cdot\|$ \\
7.7 .4 & $(0.999,0.049)$ & $(9.96 \mathrm{e}-13,-4.89 \mathrm{e}-14)$ & $9.97 \mathrm{e}-13$ & $\mathcal{Z}_{2}$ & 5725 & $\|\cdot\|$ \\
7.7 .5 & $(0.049,0.999)$ & $(-6.92 \mathrm{e}-18,4.33 \mathrm{e}-19)$ & $6.94 \mathrm{e}-18$ & $\mathcal{Z}_{2}$ & 1 & $\mathcal{Z}_{0}$ \\
7.7 .6 & $(0.000,1.000)$ & $(-4.90 \mathrm{e}-02,2.41 \mathrm{e}-03)$ & $4.91 \mathrm{e}-02$ & $\mathcal{Z}_{2}$ & 1 & $\mathcal{Z}_{0}$ \\
7.7 .7 & $(-0.999,0.049)$ & $(-9.99 \mathrm{e}-01,4.91 \mathrm{e}-02)$ & $1.00 \mathrm{e}+00$ & $\mathcal{Z}_{0}$ & 0 & $\mathcal{Z}_{0}$ \\
\hline
\end{tabular}




\section{Appendix. Theoretical aspects of the multiple impact}

In this section we recall the geometric structures and properties involved in the study of multiple impacts and the constitutive characterization of the multiple contact/impact, as presented in [1], for the particular system given by the disk in the corner. The arguments are presented in a very synthetic way, just to made the paper self consistent. For a more general and exhaustive description we refer to [1] and the references therein.

\subsection{Geometry of the system}

The geometric setup suitable to study in a frame independent and time dependent way the mechanical system formed by a rigid disk of mass $m$ having multiple contact/impact with two walls forming a corner consists in:

- a bundle $\pi_{t}: \mathcal{M} \rightarrow \mathbb{E}$, being $\mathcal{M}$ a $(3+1)$-dimensional differentiable manifold and $\mathbb{E}$ the affine time line. The elements of $\mathcal{M}$ are called space-time configurations of the system and the simplest coordinates describing $\mathcal{M}$ are the fibred coordinates $(t, x, y, \vartheta)$, where $t$ is the time coordinate, $(x, y)$ are the coordinate of the center of the disk and $\vartheta$ is the orientation of the disk;

- the first jet-extension $\pi: J(\mathcal{M}) \rightarrow \mathcal{M}$ of the bundle $\mathcal{M}$, representing the space of absolute velocities of the system. It is a $(6+1)$-dimensional affine subbundle of the tangent bundle $T(\mathcal{M})$ of $\mathcal{M}$ that can be referred to jet-coordinates $(t, x, y, \vartheta, \dot{x}, \dot{y}, \dot{\vartheta})$. Using these local coordinates, the elements of $J(\mathcal{M})$ have the form $\mathbf{p}=\frac{\partial}{\partial t}+\dot{x} \frac{\partial}{\partial x}+\dot{y} \frac{\partial}{\partial y}+\dot{\vartheta} \frac{\partial}{\partial \vartheta}$;

- the vertical vector bundle $\pi: V(\mathcal{M}) \rightarrow \mathcal{M}$ of the vectors of $T(\mathcal{M})$ that are vertical with respect to $\pi_{t}$, that is, that are tangent to the fibers of $\mathcal{M}$. The bundle $V(\mathcal{M})$ is the vector bundle modelling the affine bundle $J(\mathcal{M})$ and it represents both the space of the relative velocities of the system (once a frame of reference is assigned) and the space of possible impulses acting on the system. It can be referred to the same jet-coordinates $(t, x, y, \vartheta, \dot{x}, \dot{y}, \dot{\vartheta})$ of $J(\mathcal{M})$ and, using these local coordinates, the elements of $V(\mathcal{M})$ have the form $\mathbf{v}=v_{x} \frac{\partial}{\partial x}+$ $v_{y} \frac{\partial}{\partial y}+\omega_{\vartheta} \frac{\partial}{\partial \vartheta}$

- a positive definite scalar product $\Phi: V(\mathcal{M}) \times \mathcal{M} V(\mathcal{M}) \rightarrow \mathbb{E}$, acting on the fibers of $V(\mathcal{M})$. It is usually called the vertical metric and it takes intrinsically into account the mass properties of the system. Using again the local coordinates $(t, x, y, \vartheta)$, the vertical metric is expressed by the positive definite matrix $G=\operatorname{diag}(m, m, A)$ where $m$ is the mass of the disk and $A$ its inertia momentum. A standard calculation 
shows that, using coordinates $(t, \xi, \eta, \vartheta)$ with $\xi=k x+y, \eta=k x-y$ coordinates, the matrix expression of the vertical metric is no more diagonal and it is transformed in

$$
\Gamma=\left(\begin{array}{ccc}
\frac{m\left(1+k^{2}\right)}{4 k^{2}} & \frac{m\left(1-k^{2}\right)}{4 k^{2}} & 0 \\
\frac{m\left(1-k^{2}\right)}{4 k^{2}} & \frac{m\left(1+k^{2}\right)}{4 k^{2}} & 0 \\
0 & 0 & A
\end{array}\right) ;
$$

- the class $\mathcal{H}_{\mathcal{M}}$ of the frames of reference of the system (without any assumption of rigidity), that is the set of global fibred sections $\mathbf{h}_{\mathcal{M}}$ : $\mathcal{M} \rightarrow J(\mathcal{M})$. Using local coordinates, the elements of $\mathcal{H}_{\mathcal{M}}$ have the form $\mathbf{h}_{\mathcal{M}}=\frac{\partial}{\partial t}+H^{x} \frac{\partial}{\partial x}+H^{y} \frac{\partial}{\partial y}+H^{\vartheta} \frac{\partial}{\partial \vartheta} ;$

- the pair of additional positional constraints $\mathcal{S}_{1}, \mathcal{S}_{2}$ describing the walls of the corner. The subbundle $i_{1}: \mathcal{S}_{1} \rightarrow \mathcal{M}$ of $\mathcal{M}$ representing $\mathcal{S}_{1}$ can be described by the cartesian representation $k x-y=0$ or by the parametric representation given by the immersion $(t, x, \vartheta) \rightsquigarrow(t, x, k x, \vartheta)$. The subbundle $i_{2}: \mathcal{S}_{2} \rightarrow \mathcal{M}$ of $\mathcal{M}$ representing $\mathcal{S}_{2}$ can be described by the cartesian representation $k x+y=0$ or by the parametric representation given by the immersion $(t, x, \vartheta) \rightsquigarrow(t, x,-k x, \vartheta)$. The single constraints $\mathcal{S}_{1}, \mathcal{S}_{2}$ determine the multiple constraint $\mathcal{S}_{12}=\mathcal{S}_{1} \cap \mathcal{S}_{2}$ where $i_{12}: \mathcal{S}_{12} \rightarrow \mathcal{M}$ is the subbundle of $\mathcal{M}$ described by the cartesian representation $x=y=0$ or by the parametric representation given by the immersion $(t, \vartheta) \rightsquigarrow(t, 0,0, \vartheta)$. The system is in contact with one or both the constraints if its space-time configuration belongs to $\mathcal{S}_{1}, \mathcal{S}_{2}$ or $\mathcal{S}_{12}$. Each of the subbundles $\mathcal{S}_{1}, \mathcal{S}_{2}, \mathcal{S}_{12}$ determines its first jet bundle $J\left(\mathcal{S}_{1}\right), J\left(\mathcal{S}_{2}\right), J\left(\mathcal{S}_{12}\right)$ of the absolute velocities tangent respectively to $\mathcal{S}_{1}, \mathcal{S}_{2}, \mathcal{S}_{12}$ (all of them affine subbundles of $J(\mathcal{M})$ ), and its vertical vector bundle $V\left(\mathcal{S}_{1}\right), V\left(\mathcal{S}_{2}\right), V\left(\mathcal{S}_{12}\right)$ (all of them vector subbundles of $V(\mathcal{M}))$;

- the so called contact bundles

$$
\begin{aligned}
& i_{1}^{*}(J(\mathcal{M})) \quad \hookrightarrow \quad J(\mathcal{M}) \\
& i_{2}^{*}(J(\mathcal{M})) \quad \hookrightarrow \quad J(\mathcal{M}) \\
& i_{12}^{*}(J(\mathcal{M})) \hookrightarrow J(\mathcal{M}) \\
& i_{1}^{*}(V(\mathcal{M})) \quad \hookrightarrow \quad V(\mathcal{M}) \\
& i_{2}^{*}(V(\mathcal{M})) \quad \hookrightarrow \quad V(\mathcal{M}) \\
& i_{12}^{*}(V(\mathcal{M})) \hookrightarrow V(\mathcal{M}) \text {, }
\end{aligned}
$$

pull-back bundles of the absolute velocities $J(\mathcal{M})$ and impulses $V(\mathcal{M})$ respectively on $\mathcal{S}_{1}, \mathcal{S}_{2}, \mathcal{S}_{12}$ and representing all the possible absolute 
velocities and impulses of the system when the system is in contact with respectively $\mathcal{S}_{1}, \mathcal{S}_{2}, \mathcal{S}_{12}$. Of course, being $\mathcal{S}_{12}=\mathcal{S}_{1} \cap \mathcal{S}_{2}$, we have $i_{12}^{*}(J(\mathcal{M}))=i_{1}^{*}(J(\mathcal{M})) \cap i_{2}^{*}(J(\mathcal{M}))$ and $i_{12}^{*}(V(\mathcal{M}))=i_{1}^{*}(V(\mathcal{M})) \cap$ $i_{2}^{*}(V(\mathcal{M}))$. Since we suppose the disk in contact with both $\mathcal{S}_{1}, \mathcal{S}_{2}$, then the geometric context where the multiple contact/impact of the disk can be framed is given by $i_{12}^{*}(J(\mathcal{M}))$ and $i_{12}^{*}(V(\mathcal{M}))$;

- the set of projection operators determined by the vertical metric $\Phi$. In particular, with obvious notation, these projection operators determine natural splits

$$
\begin{aligned}
& i_{1}^{*}(V(\mathcal{M}))=V\left(\mathcal{S}_{1}\right) \oplus V^{\perp}\left(\mathcal{S}_{1}\right) \Leftrightarrow \mathbf{v}=\mathbf{v}_{1}^{\|}+\mathbf{v}_{1}^{\perp}(\mathbf{p}) \\
& i_{2}^{*}(V(\mathcal{M}))=V\left(\mathcal{S}_{2}\right) \oplus V^{\perp}\left(\mathcal{S}_{2}\right) \Leftrightarrow \mathbf{v}=\mathbf{v}_{2}^{\|}+\mathbf{v}_{2}^{\perp}(\mathbf{p}) \\
& i_{12}^{*}(V(\mathcal{M}))=V\left(\mathcal{S}_{12}\right) \oplus V^{\perp}\left(\mathcal{S}_{12}\right) \Leftrightarrow \mathbf{v}=\mathbf{v}_{12}^{\|}+\mathbf{v}_{12}^{\perp}(\mathbf{p}) \\
& i_{1}^{*}(J(\mathcal{M}))=J\left(\mathcal{S}_{1}\right) \oplus V^{\perp}\left(\mathcal{S}_{1}\right) \Leftrightarrow \mathbf{p}=\mathbf{p}_{1}+\mathbf{v}_{1}^{\perp}(\mathbf{p}) \\
& i_{2}^{*}(J(\mathcal{M}))=J\left(\mathcal{S}_{2}\right) \oplus V^{\perp}\left(\mathcal{S}_{2}\right) \Leftrightarrow \mathbf{p}=\mathbf{p}_{2}+\mathbf{v}_{2}^{\perp}(\mathbf{p}) \\
& i_{12}^{*}(J(\mathcal{M}))=J\left(\mathcal{S}_{12}\right) \oplus V^{\perp}\left(\mathcal{S}_{12}\right) \Leftrightarrow \mathbf{p}=\mathbf{p}_{12}+\mathbf{v}_{12}^{\perp}(\mathbf{p}) \text {. }
\end{aligned}
$$

Being $\mathcal{S}_{1}, \mathcal{S}_{2}$ of codimension 1 , also the orthogonal vector subbundles $V^{\perp}\left(\mathcal{S}_{1}\right), V^{\perp}\left(\mathcal{S}_{2}\right)$ has codimension 1 , so that we can introduce two vertical vectors $\mathbf{u}_{1}^{\perp}, \mathbf{u}_{2}^{\perp}$ such that $V^{\perp}\left(\mathcal{S}_{1}\right)=\operatorname{Lin}\left(\mathbf{u}_{1}^{\perp}\right), V^{\perp}\left(\mathcal{S}_{2}\right)=\operatorname{Lin}\left(\mathbf{u}_{2}^{\perp}\right)$. The impact (with $\mathcal{S}_{1}, \mathcal{S}_{2}$ or both) nature of an absolute velocity $\mathbf{p} \in$ $i_{12}^{*}(J(\mathcal{M}))$ is determined by the (suitably chosen) sign of the scalar products $\Phi\left(\mathbf{v}_{1}^{\perp}(\mathbf{p}), \mathbf{u}_{1}^{\perp}\right), \Phi\left(\mathbf{v}_{2}^{\perp}(\mathbf{p}), \mathbf{u}_{2}^{\perp}\right)$;

- the subclasses $\mathcal{H}_{\mathcal{S}_{1}}, \mathcal{H}_{\mathcal{S}_{2}}, \mathcal{H}_{\mathcal{S}_{12}}$ of the frames of reference of $\mathcal{H}_{\mathcal{M}}$ that are tangent to $\mathcal{S}_{1}, \mathcal{S}_{2}, \mathcal{S}_{12}$ respectively. Using local coordinates, the elements of $\mathcal{H}_{\mathcal{S}_{1}}, \mathcal{H}_{\mathcal{S}_{2}}, \mathcal{H}_{\mathcal{S}_{12}}$ have the forms

$$
\begin{array}{r}
\mathbf{h}_{\mathcal{S}_{1}}=\frac{\partial}{\partial t}+H^{x} \frac{\partial}{\partial x}+k H^{x} \frac{\partial}{\partial y}+H^{\vartheta} \frac{\partial}{\partial \vartheta} \\
\mathbf{h}_{\mathcal{S}_{2}=} \frac{\partial}{\partial t}+H^{x} \frac{\partial}{\partial x}-k H^{x} \frac{\partial}{\partial y}+H^{\vartheta} \frac{\partial}{\partial \vartheta} \\
\mathbf{h}_{\mathcal{S}_{12}=} \frac{\partial}{\partial t} \quad+H^{\vartheta} \frac{\partial}{\partial \vartheta}
\end{array}
$$

The three subclasses represent the set of frames that can be considered at rest with $\mathcal{S}_{1}, \mathcal{S}_{2}, \mathcal{S}_{12}$ respectively, that is the sole frames for which the conservation of kinetic energy could have an invariant meaning (see [10, 1]).

\subsection{Constitutive law for ideal impact}

The frame independent description of an impulsive dynamic problem in this geometric context consists in determining an element $\mathbf{p}_{R} \in i_{12}^{*}(J(\mathcal{M}))$, 
the right-velocity, once an element $\mathbf{p}_{L} \in i_{12}^{*}(J(\mathcal{M}))$, the left-velocity, is known. Taking into account the action of $V(\mathcal{M})$ as modelling vector bundle of the affine bundle $J(\mathcal{M})$, this is equivalent to the assignment of an impulse $\mathbf{I}=\mathbf{I}\left(\mathbf{p}_{L}\right) \in i_{12}^{*}(V(\mathcal{M}))$ such that $\mathbf{p}_{R}=\mathbf{p}_{L}+\mathbf{I}\left(\mathbf{p}_{L}\right)$. A constitutive law is then an assignment

$$
\begin{aligned}
\mathbf{I}_{\text {const }}: i_{12}^{*}(J(\mathcal{M})) & \rightarrow i_{12}^{*}(V(\mathcal{M})) \\
\mathbf{p}_{L} & \rightsquigarrow \mathbf{I}_{\text {const }}\left(\mathbf{p}_{L}\right) .
\end{aligned}
$$

The ideal constitutive law for multiple impact presented in ([1]) is based on three assumptions:

a) the preservation of kinetic energy of the system before and after the contact/impact with the constraints in every frame of reference for which the requirement has a clear meaning;

b) in absence of additional information about the constraints, the constraints involved in a multiple contact/impact cannot be discerned;

c) in case of contact of the disk with both the constraints $\mathcal{S}_{1}, \mathcal{S}_{2}$ but of impact with only one of the constraints, the constitutive characterization must coincide with the usual ideal characterization of a single constraint.

Let the coefficient $\lambda_{\text {ideal }}$ be defined by

$$
\lambda_{\text {ideal }}=-2 \frac{\Phi\left(\mathbf{v}_{12}^{\perp}\left(\mathbf{p}_{L}\right), \mathbf{v}_{1}^{\perp}\left(\mathbf{p}_{L}\right)+\mathbf{v}_{2}^{\perp}\left(\mathbf{p}_{L}\right)\right)}{\Phi\left(\mathbf{v}_{1}^{\perp}\left(\mathbf{p}_{L}\right)+\mathbf{v}_{2}^{\perp}\left(\mathbf{p}_{L}\right), \mathbf{v}_{1}^{\perp}\left(\mathbf{p}_{L}\right)+\mathbf{v}_{2}^{\perp}\left(\mathbf{p}_{L}\right)\right)}:
$$

recalling that the three assumption listed above are insufficient to determine univocally the constitutive characterization in case of multiple contacts/impacts, the simplest non trivial ideal constitutive characterization of possible multiple impacts presented in [1] applied to the disk in the corner is:

$$
\begin{array}{lll}
\text { no impact } & \Rightarrow \mathbf{I}\left(\mathbf{p}_{L}\right)=0 & \Leftrightarrow \mathbf{p}=\mathbf{p}_{L}=\mathbf{p}_{R} \\
\text { impact with } \mathcal{S}_{1} & \Rightarrow \mathbf{I}\left(\mathbf{p}_{L}\right)=-2 \mathbf{v}_{1}^{\perp}\left(\mathbf{p}_{L}\right) & \Leftrightarrow \mathbf{p}=\mathbf{p}_{L}-2 \mathbf{v}_{1}^{\perp}\left(\mathbf{p}_{L}\right) \\
\text { impact with } \mathcal{S}_{2} & \Rightarrow \mathbf{I}\left(\mathbf{p}_{L}\right)=-2 \mathbf{v}_{2}^{\perp}\left(\mathbf{p}_{L}\right) & \Leftrightarrow \mathbf{p}=\mathbf{p}_{L}-2 \mathbf{v}_{2}^{\perp}\left(\mathbf{p}_{L}\right) \\
\text { multiple impact } & \Rightarrow \mathbf{I}\left(\mathbf{p}_{L}\right)=\lambda\left(\mathbf{v}_{1}^{\perp}\left(\mathbf{p}_{L}\right)+\mathbf{v}_{2}^{\perp}\left(\mathbf{p}_{L}\right)\right) & \Leftrightarrow \mathbf{p}=\mathbf{p}_{L}+\lambda_{\text {ideal }}\left(\mathbf{v}_{1}^{\perp}\left(\mathbf{p}_{L}\right)+\mathbf{v}_{2}^{\perp}\left(\mathbf{p}_{L}\right)\right)
\end{array}
$$

In complete analogy, let $\varepsilon \in[0,1)$ and let the coefficient $\lambda_{\text {non-ideal }}$ be defined by

$$
\lambda_{\text {non-ideal }}=-(1+\varepsilon) \frac{\Phi\left(\mathbf{v}_{12}^{\perp}\left(\mathbf{p}_{L}\right), \mathbf{v}_{1}^{\perp}\left(\mathbf{p}_{L}\right)+\mathbf{v}_{2}^{\perp}\left(\mathbf{p}_{L}\right)\right)}{\Phi\left(\mathbf{v}_{1}^{\perp}\left(\mathbf{p}_{L}\right)+\mathbf{v}_{2}^{\perp}\left(\mathbf{p}_{L}\right), \mathbf{v}_{1}^{\perp}\left(\mathbf{p}_{L}\right)+\mathbf{v}_{2}^{\perp}\left(\mathbf{p}_{L}\right)\right)}:
$$


the simplest non trivial non-ideal constitutive characterization of possible multiple impacts applied to the disk in the corner is:

$$
\begin{array}{lll}
\text { no impact } & \Rightarrow \mathbf{I}\left(\mathbf{p}_{L}\right)=0 & \Leftrightarrow \mathbf{p}=\mathbf{p}_{L}=\mathbf{p}_{R} \\
\text { impact with } \mathcal{S}_{1} \Rightarrow \mathbf{I}\left(\mathbf{p}_{L}\right)=-2 \mathbf{v}_{1}^{\perp}\left(\mathbf{p}_{L}\right) & \Leftrightarrow \mathbf{p}=\mathbf{p}_{L}-(1+\varepsilon) \mathbf{v}_{1}^{\perp}\left(\mathbf{p}_{L}\right) \\
\text { impact with } \mathcal{S}_{2} \Rightarrow \mathbf{I}\left(\mathbf{p}_{L}\right)=-2 \mathbf{v}_{2}^{\perp}\left(\mathbf{p}_{L}\right) & \Leftrightarrow \mathbf{p}=\mathbf{p}_{L}-(1+\varepsilon) \mathbf{v}_{2}^{\perp}\left(\mathbf{p}_{L}\right) \\
\text { multiple impact } & \Rightarrow \mathbf{I}\left(\mathbf{p}_{L}\right)=\lambda\left(\mathbf{v}_{1}^{\perp}\left(\mathbf{p}_{L}\right)+\mathbf{v}_{2}^{\perp}\left(\mathbf{p}_{L}\right)\right) & \Leftrightarrow \mathbf{p}=\mathbf{p}_{L}+\lambda_{\text {non-ideal }}\left(\mathbf{v}_{1}^{\perp}\left(\mathbf{p}_{L}\right)+\mathbf{v}_{2}^{\perp}\left(\mathbf{p}_{L}\right)\right)
\end{array}
$$

It is however clear that the assignment of a new velocity $\mathbf{p}_{n e w}=\mathbf{p}_{L}+$ $\mathbf{I}\left(\mathbf{p}_{L}\right)$ with the rule described above does not ensure that the system subject to $\mathbf{p}_{\text {new }}$ does not impact again with the constraints. It is then necessary to construct an iterative procedure that applies the rule until the velocity obtained does not give an impact with one or both the constraints and can be considered the right velocity $\mathbf{p}_{R}$ of the system after the impact. This of course opens the problem of the termination analysis of the algorithm discussed above.

\subsection{Coordinate expressions of the iterative rule}

For the convenience of the Reader, we list now the local coordinate expressions (using the cartesian representation of $\mathcal{S}_{1}, \mathcal{S}_{2}$ in the fibred coordinates $(t, x, y, \vartheta)$ for $\mathcal{M})$ of the main objects described in this appendix and used to obtain the iterative rule described in the paper. Taking into account that we focus our attention to multiple contact/impact, let $\mathbf{p} \in i_{12}^{*}(J(\mathcal{M}))$ be an absolute velocity of the system when the system is in contact with both $\mathcal{S}_{1}, \mathcal{S}_{2}$ (so that $\pi(\mathbf{p}) \in \mathcal{S}_{12}$ ). Then:

$$
\begin{aligned}
& \mathbf{p}=\frac{\partial}{\partial t}+\dot{x} \frac{\partial}{\partial x}+\dot{y} \frac{\partial}{\partial y}+\dot{\vartheta} \frac{\partial}{\partial \vartheta} \\
& \mathbf{v}_{1}^{\perp}(\mathbf{p})=\frac{k}{1+k^{2}}(k \dot{x}-\dot{y}) \frac{\partial}{\partial x}-\frac{1}{1+k^{2}}(k \dot{x}-\dot{y}) \frac{\partial}{\partial y} \\
& \mathbf{v}_{2}^{\perp}(\mathbf{p})=\frac{k}{1+k^{2}}(k \dot{x}+\dot{y}) \frac{\partial}{\partial x}+\frac{1}{1+k^{2}}(k \dot{x}+\dot{y}) \frac{\partial}{\partial y} \\
& \mathbf{v}_{12}^{\perp}(\mathbf{p})=\dot{x} \frac{\partial}{\partial x}+\dot{y} \frac{\partial}{\partial y} ; \\
& \mathbf{u}_{1}^{\perp}=\frac{k}{1+k^{2}} \frac{\partial}{\partial x}-\frac{1}{1+k^{2}} \frac{\partial}{\partial y} \\
& \mathbf{u}_{2}^{\perp}=\frac{k}{1+k^{2}} \frac{\partial}{\partial x}+\frac{1}{1+k^{2}} \frac{\partial}{\partial y}
\end{aligned}
$$




$$
\begin{aligned}
& \Phi\left(\mathbf{v}_{1}^{\perp}(\mathbf{p}), \mathbf{u}_{1}^{\perp}\right)=\frac{m}{1+k^{2}}(k \dot{x}-\dot{y}) \\
& \Phi\left(\mathbf{v}_{2}^{\perp}(\mathbf{p}), \mathbf{u}_{2}^{\perp}\right)=\frac{m}{1+k^{2}}(k \dot{x}+\dot{y})
\end{aligned}
$$

The coordinates expressions of the various geometrical objects describing the problem and the rules assigning the constitutive characterization of the contact/impact suggest two simple remarks:

1) since the coordinate expressions (22) do not involve terms pertaining $\frac{\partial}{\partial \vartheta}$, and the rules (19/20) assigning the absolute velocity after the impact involves only the orthogonal velocities (22), then the component along $\frac{\partial}{\partial \vartheta}$ of $\mathbf{p}$ is not changed by the impact. This is coherent with the absence of friction of the disk with $\mathcal{S}_{1}$ and $\mathcal{S}_{2}$ implied by the ideality of the contact. Moreover, this justifies the fact that the iterative rule can then be expressed using the coordinates $\dot{x}, \dot{y}$ alone;

2) taking into account Fig. 1, the conditions for $\mathbf{p}$ to be an impact velocity for $\mathcal{S}_{1}, \mathcal{S}_{2}$ are then

$$
\Phi\left(\mathbf{v}_{1}^{\perp}(\mathbf{p}), \mathbf{u}_{1}^{\perp}\right)>0, \quad \Phi\left(\mathbf{v}_{2}^{\perp}(\mathbf{p}), \mathbf{u}_{2}^{\perp}\right)>0
$$

respectively. Together with (24), this justifies the description of the zones $\mathcal{Z}_{0}, \mathcal{Z}_{1}, \mathcal{Z}_{2}, \mathcal{Z}_{12}$ in terms of the signs of $k \dot{x}+\dot{y}, k \dot{x}-\dot{y}$.

\section{References}

[1] S. Pasquero. Ideal characterizations of multiple impacts: A frame independent approach by means of jet bundle geometry. Quarterly of Applied Mathematics, volume 76, pages 547-576, 2018.

[2] B. Brogliato. Nonsmooth Impact Mechanics. Models, Dynamics and Control - Third Edition. Springer International Publishing Switzerland; Communications and Control Engineering, 2016.

[3] Bernard Brogliato. Impacts in mechanical systems: analysis and modelling, volume 551. Springer Science \& Business Media, 2000.

[4] Friedrich Pfeiffer and Christoph Glocker. Multibody dynamics with unilateral contacts, volume 421. Springer Science \& Business Media, 2000.

[5] Caishan Liu, Zhen Zhao, and Bernard Brogliato. Frictionless multiple impacts in multibody systems. i. theoretical framework. In Proceedings of the Royal Society of London A: Mathematical, Physical and Engineering Sciences, volume 464, pages 3193-3211. The Royal Society, 2008. 
[6] K.L. Johnson. Contact mechanics. Cambridge University Press, Cambridge, 1985.

[7] W.J. Stronge. Impact Mechanics. Cambridge University Press., Cambridge, 2000.

[8] E. Isaacson and H.B. Keller. Analysis of Numerical Methods. Dover, New York, 1994.

[9] Peter D. Lax. Functional Analysis. Wiley-Interscience, 2002.

[10] S. Pasquero. Ideality criterion for unilateral constraints in timedependent impulsive mechanics. Journ. Math. Phys., 46,n.11:11290420pp, 2005. 